Supplementary Information for:

\title{
Insertion of $\mathrm{CO}_{2}$, ketones, and aldehydes into the $\mathrm{C}$-Li bond of 1,3,5-triaza-7- phosphaadamantan-6-yllithium (PTA-Li)
}

\author{
Gene W. Wong, Wei-Chih Lee, and Brian J. Frost*
}

\author{
1-S Table of Contents \\ 2-S Figure 1-S. ${ }^{1} \mathrm{H}$ NMR spectrum of PTA- $\mathrm{CO}_{2} \mathrm{Li}$ in $\mathrm{D}_{2} \mathrm{O}$. \\ Figure 2-S. ${ }^{31} \mathrm{P}\left\{{ }^{1} \mathrm{H}\right\}$ NMR spectrum of PTA- $\mathrm{CO}_{2} \mathrm{Li}$ in $\mathrm{D}_{2} \mathrm{O}$. \\ 3-S Figure $3-\mathrm{S} .{ }^{31} \mathrm{P}\left\{{ }^{1} \mathrm{H}\right\}$ NMR spectrum of $\mathrm{O}=\mathrm{PTA}-\mathrm{CO}_{2} \mathrm{Li}$ in $\mathrm{D}_{2} \mathrm{O}$. \\ Experimental details for synthesis of PTA- ${ }^{13} \mathrm{CO}_{2} \mathrm{Li}$ \\ Scheme S-1. Proposed synthesis of PTA- $\left(\mathrm{CO}_{2} \mathrm{Li}\right)_{2}$. \\ 4-S Figure 4-S. ESI mass spectra of PTA-CO $\mathrm{CO}_{2} \mathrm{Li}$ and PTA- ${ }^{13} \mathrm{CO}_{2} \mathrm{Li}$. \\ Figure 5-S. ${ }^{31} \mathrm{P}\left\{{ }^{1} \mathrm{H}\right\}$ NMR spectrum of PTA- ${ }^{13} \mathrm{CO}_{2} \mathrm{Li}$ in $\mathrm{CD}_{3} \mathrm{OD}$. \\ 5-S Figure 6-S. ${ }^{13} \mathrm{C} \mathrm{NMR}$ spectrum of PTA- ${ }^{13} \mathrm{CO}_{2} \mathrm{Li}$ in $\mathrm{CD}_{3} \mathrm{OD}$. \\ Figure 7-S. ${ }^{1} \mathrm{H}$ NMR spectrum of PTA- $\mathrm{CO}_{2} \mathrm{Me}$ in $\mathrm{CDCl}_{3}$ \\ 6-S Figure 8-S. ${ }^{31} \mathrm{P}\left\{{ }^{1} \mathrm{H}\right\} \mathrm{NMR}$ spectrum of PTA- $\mathrm{CO}_{2} \mathrm{Me}$ in $\mathrm{CDCl}_{3}$. \\ Figure 9-S. ${ }^{31} \mathrm{P}\left\{{ }^{1} \mathrm{H}\right\}$ NMR spectrum of $\mathrm{O}=\mathrm{PTA}-\mathrm{CO}_{2} \mathrm{Me}$ in $\mathrm{D}_{2} \mathrm{O}$. \\ 7-S Figure 10-S. ${ }^{1} \mathrm{H}$ NMR spectrum of PTA-C $\left(\mathrm{C}_{6} \mathrm{H}_{5}\right)_{2} \mathrm{OH}$ in $\mathrm{CDCl}_{3}$. \\ Figure 11-S. ${ }^{31} \mathrm{P}\left\{{ }^{1} \mathrm{H}\right\}$ NMR spectrum of PTA-C $\left(\mathrm{C}_{6} \mathrm{H}_{5}\right)_{2} \mathrm{OH}$ in $\mathrm{CDCl}_{3}$. \\ 8-S Figure 12-S. ${ }^{31} \mathrm{P}\left\{{ }^{1} \mathrm{H}\right\}$ NMR spectrum of $\mathrm{O}=\mathrm{PTA}-\mathrm{C}\left(\mathrm{C}_{6} \mathrm{H}_{5}\right)_{2} \mathrm{OH}$ in $\mathrm{D}_{2} \mathrm{O}$. \\ Figure 13-S. ${ }^{1} \mathrm{H}$ NMR spectrum of PTA-C $\left(\mathrm{C}_{6} \mathrm{H}_{4} \mathrm{OCH}_{3}\right)_{2} \mathrm{OH}$ in $\mathrm{CDCl}_{3}$. \\ 9-S Figure 14-S. ${ }^{31} \mathrm{P}\left\{{ }^{1} \mathrm{H}\right\}$ NMR spectrum of PTA-C $\left(\mathrm{C}_{6} \mathrm{H}_{4} \mathrm{OCH}_{3}\right)_{2} \mathrm{OH}$ in $\mathrm{CDCl}_{3}$. \\ Figure 15-S. ${ }^{31} \mathrm{P}\left\{{ }^{1} \mathrm{H}\right\}$ NMR spectrum of $\mathrm{O}=\mathrm{PTA}-\mathrm{C}\left(\mathrm{C}_{6} \mathrm{H}_{4} \mathrm{OCH}_{3}\right)_{2} \mathrm{OH}$ in $\mathrm{D}_{2} \mathrm{O}$. \\ 10-S Figure 16-S. ${ }^{1} \mathrm{H}$ NMR spectrum of PTA-CH $\left(\mathrm{C}_{6} \mathrm{H}_{4} \mathrm{OCH}_{3}\right) \mathrm{OH}$ in $\mathrm{CDCl}_{3}$. \\ Figure 17-S. ${ }^{31} \mathrm{P}\left\{{ }^{1} \mathrm{H}\right\}$ NMR spectrum of PTA- $\mathrm{CH}\left(\mathrm{C}_{6} \mathrm{H}_{4} \mathrm{OCH}_{3}\right) \mathrm{OH}$ in $\mathrm{CDCl}_{3}$. \\ 11-S Figure 18-S. ${ }^{31} \mathrm{P}\left\{{ }^{1} \mathrm{H}\right\}$ NMR spectrum of $\mathrm{O}=\mathrm{PTA}-\mathrm{CH}\left(\mathrm{C}_{6} \mathrm{H}_{4} \mathrm{OCH}_{3}\right) \mathrm{OH}$ in $\mathrm{D}_{2} \mathrm{O}$. \\ Figure 19-S. ${ }^{1} \mathrm{H}$ NMR spectrum of PTA-CH(ferrocenyl)OH in $\mathrm{CDCl}_{3}$. \\ 12-S Figure 20-S. ${ }^{31} \mathrm{P}\left\{{ }^{1} \mathrm{H}\right\}$ NMR spectrum of PTA-CH(ferrocenyl)OH in $\mathrm{CDCl}_{3}$. \\ Figure 21-S. ${ }^{31} \mathrm{P}\left\{{ }^{1} \mathrm{H}\right\}$ NMR spectrum of $\mathrm{O}=\mathrm{PTA}-\mathrm{CH}\left(\right.$ ferrocenyl) $\mathrm{OH}$ in $\mathrm{D}_{2} \mathrm{O}$. \\ 13-S Figure 22-S. ${ }^{1} \mathrm{H}$ NMR spectrum of $\left(\eta^{6}-\mathrm{C}_{6} \mathrm{H}_{5} \mathrm{CH}_{3}\right) \mathrm{RuCl}_{2}\left(\mathrm{PTA}-\mathrm{C}\left(\mathrm{C}_{6} \mathrm{H}_{5}\right)_{2} \mathrm{OH}\right)$ in $\mathrm{CDCl}_{3}$. \\ Figure 23-S. ${ }^{31} \mathrm{P}\left\{{ }^{1} \mathrm{H}\right\}$ NMR spectrum of $\left(\eta^{6}-\mathrm{C}_{6} \mathrm{H}_{5} \mathrm{CH}_{3}\right) \mathrm{RuCl}_{2}\left(\mathrm{PTA}-\mathrm{C}\left(\mathrm{C}_{6} \mathrm{H}_{5}\right)_{2} \mathrm{OH}\right)$ in $\mathrm{CDCl}_{3}$. \\ 14-S Figure 24-S. ${ }^{1} \mathrm{H}$ NMR spectrum of $\left(\eta^{6}-\mathrm{C}_{6} \mathrm{H}_{6}\right) \mathrm{RuCl}_{2}\left(\mathrm{PTA}-\mathrm{C}\left(\mathrm{C}_{6} \mathrm{H}_{4} \mathrm{OCH}_{3}\right)_{2} \mathrm{OH}\right)$ in $\mathrm{CDCl}_{3}$. \\ Figure 25-S. ${ }^{31} \mathrm{P}\left\{{ }^{1} \mathrm{H}\right\}$ NMR spectrum of $\left(\eta^{6}-\mathrm{C}_{6} \mathrm{H}_{6}\right) \mathrm{RuCl}_{2}\left(\mathrm{PTA}-\mathrm{C}\left(\mathrm{C}_{6} \mathrm{H}_{4} \mathrm{OCH}_{3}\right)_{2} \mathrm{OH}\right)$ in $\mathrm{CDCl}_{3}$. \\ 15-S Figure 26-S. ${ }^{31} \mathrm{P}\left\{{ }^{1} \mathrm{H}\right\}$ NMR spectrum of $\left(\eta^{6}-\mathrm{C}_{6} \mathrm{H}_{6}\right) \mathrm{RuCl}_{2}\left(\mathrm{PTA}-\mathrm{CH}\left(\mathrm{C}_{6} \mathrm{H}_{4} \mathrm{OCH}_{3}\right) \mathrm{OH}\right)$ in $\mathrm{CDCl}_{3}$. \\ Figure 27-S. Ball and stick representation of a $\kappa^{1}(\mathrm{P})-\kappa^{2}(\mathrm{~N}, \mathrm{O})$ binding mode of [PTA-CH(ferrocenyl)O- \\ 16-S Table 1-S. Bond lengths $[\AA]$ and angles $\left[{ }^{\circ}\right]$ for $\mathrm{O}=\mathrm{PTA}-\mathrm{CO}_{2} \mathrm{Me}$. \\ 17-S Table 2-S. Bond lengths $[\AA]$ and angles $\left[{ }^{\circ}\right]$ for PTA-C $\left(\mathrm{C}_{6} \mathrm{H}_{5}\right)_{2} \mathrm{OH}$. \\ 20-S Table 3-S. Bond lengths $[\AA]$ and angles $\left[^{\circ}\right]$ for $\mathrm{O}=\mathrm{PTA}-\mathrm{C}\left(\mathrm{C}_{6} \mathrm{H}_{4} \mathrm{OCH}_{3}\right)_{2} \mathrm{OH}$. \\ 22 -S Table 4-S. Hydrogen bonds for $\mathrm{O}=\mathrm{PTA}-\mathrm{C}\left(\mathrm{C}_{6} \mathrm{H}_{4} \mathrm{OCH}_{3}\right)_{2} \mathrm{OH}\left[\AA\right.$ and $\left.{ }^{\circ}\right]$. \\ Table 5-S. Bond lengths $[\AA]$ and angles $\left[^{\circ}\right]$ for $\mathrm{O}=\mathrm{PTA}-\mathrm{CH}\left(\mathrm{C}_{6} \mathrm{H}_{4} \mathrm{OCH}_{3}\right) \mathrm{OH}$. \\ 23-S Table 6-S. Hydrogen bonds for $\mathrm{O}=\mathrm{PTA}-\mathrm{CH}\left(\mathrm{C}_{6} \mathrm{H}_{4} \mathrm{OCH}_{3}\right) \mathrm{OH}\left[\AA\right.$ and $\left.^{\circ}\right]$. \\ 24-S Table 7-S. Bond lengths [Å] and angles [ [ ${ }^{\circ}$ for PTA-CH(ferrocenyl)OH. \\ 26-S Table 8-S. Hydrogen bonds for PTA-CH(ferrocenyl)OH [ $\AA^{\circ}$ and $\left.{ }^{\circ}\right]$. \\ Table 9-S. Bond lengths $[\AA]$ and angles $\left[^{\circ}\right]$ for $\left[\left(\eta^{6}-\mathrm{C}_{6} \mathrm{H}_{5} \mathrm{CH}_{3}\right) \mathrm{Ru}\left(\mathrm{PTA}-\mathrm{C}\left(\mathrm{C}_{6} \mathrm{H}_{5}\right)_{2} \mathrm{OH}\right) \mathrm{Cl}_{2}\right]$. \\ 28-S Table 10-S. Hydrogen bonds for $\left.\left[\left(\eta^{6}-\mathrm{C}_{6} \mathrm{H}_{5} \mathrm{CH}_{3}\right) \mathrm{Ru}\left(\mathrm{PTA}-\mathrm{C}\left(\mathrm{C}_{6} \mathrm{H}_{5}\right)_{2} \mathrm{OH}\right) \mathrm{Cl}_{2}\right)\right]$. \\ Table 11-S. Bond lengths $[\AA]$ and angles $\left[^{\circ}\right]$ for $\left(\eta^{6}-\mathrm{C}_{6} \mathrm{H}_{6}\right) \mathrm{RuCl}_{2}\left(\mathrm{PTA}-\mathrm{C}\left(\mathrm{C}_{6} \mathrm{H}_{4} \mathrm{OCH}_{3}\right)_{2} \mathrm{OH}\right)$. \\ 33-S Table 12-S. Hydrogen bonds for $\left(\eta^{6}-\mathrm{C}_{6} \mathrm{H}_{6}\right) \mathrm{RuCl}_{2}\left(\mathrm{PTA}-\mathrm{C}\left(\mathrm{C}_{6} \mathrm{H}_{4} \mathrm{OCH}_{3}\right)_{2} \mathrm{OH}\right)\left[\AA\right.$ and $\left.{ }^{\circ}\right]$. \\ Table 13-S. Bond lengths $[\AA]$ and angles $\left[{ }^{\circ}\right]$ for $\left[\left(\eta^{6}-\mathrm{C}_{6} \mathrm{H}_{6}\right) \mathrm{Ru}\left(\mathrm{PTA}-\mathrm{CH}\left(\mathrm{C}_{6} \mathrm{H}_{4} \mathrm{OCH}_{3}\right) \mathrm{OH}\right) \mathrm{Cl}_{2}\right]$.
}


Figure 1-S. ${ }^{1} \mathrm{H}$ NMR spectrum of $\mathrm{PTA}-\mathrm{CO}_{2} \mathrm{Li}$ in $\mathrm{D}_{2} \mathrm{O}$.

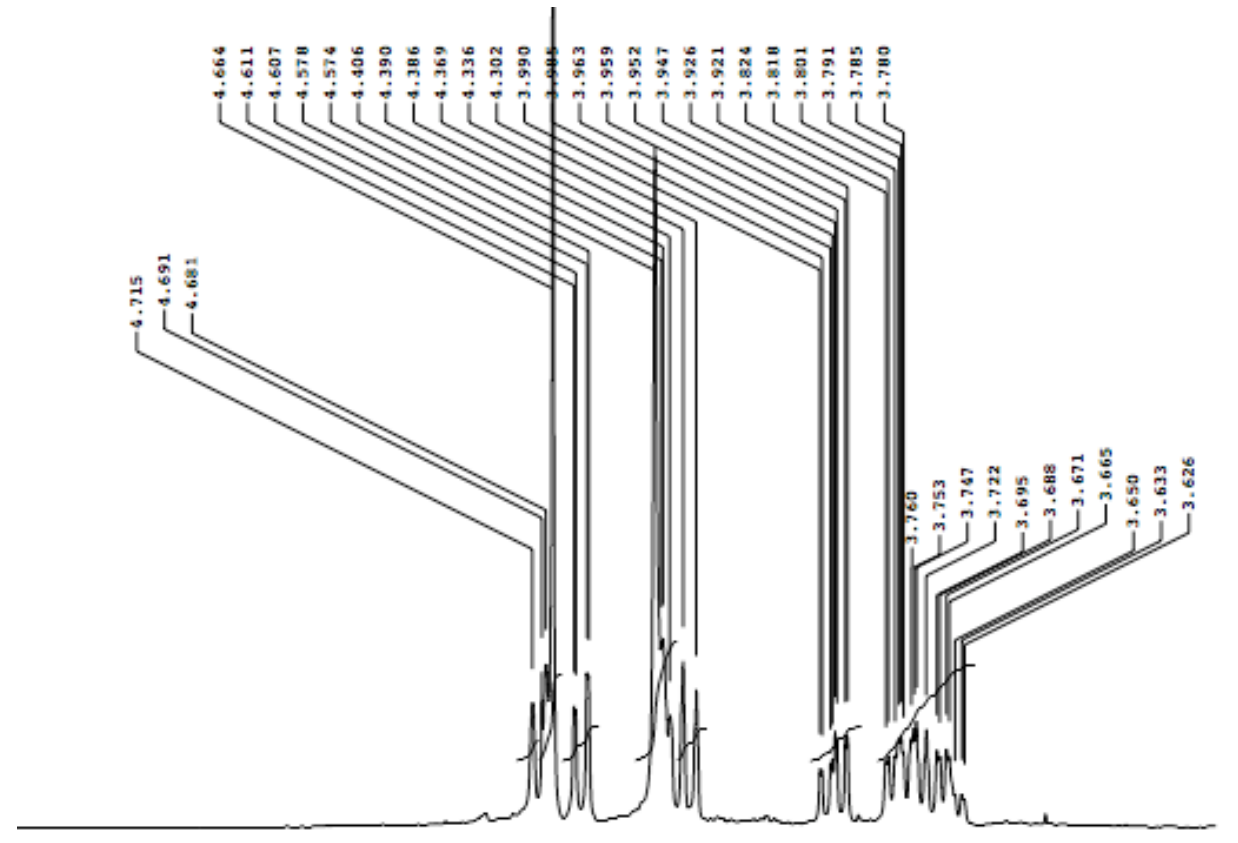

प

$\begin{array}{llllllllllllll}5.8 & 5.6 & 5.4 & 5.2 & 5.0 & 4.8 & 4.6 & 4.4 & 4.2 & 4.0 & 3.8 & 3.6 & 3.4 & \mathrm{ppm}\end{array}$

Figure 2-S. ${ }^{31} \mathrm{P}$ NMR spectrum of PTA- $\mathrm{CO}_{2} \mathrm{Li}$ in $\mathrm{D}_{2} \mathrm{O}$.

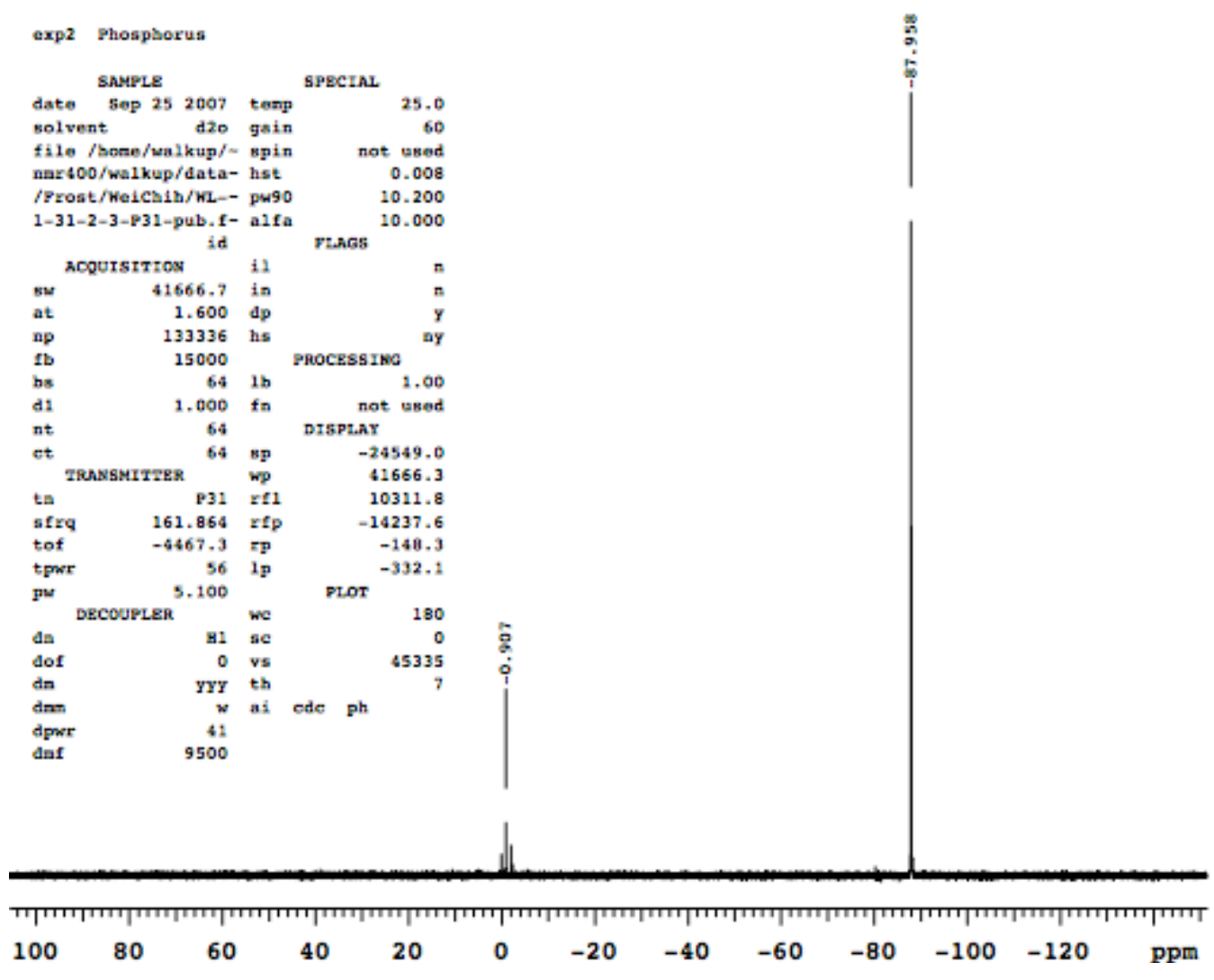


Figure 3-S. ${ }^{31} \mathrm{P}\left\{{ }^{1} \mathrm{H}\right\}$ NMR spectrum of $\mathrm{O}=\mathrm{PTA}-\mathrm{CO}_{2} \mathrm{Li}$ in $\mathrm{D}_{2} \mathrm{O}$.

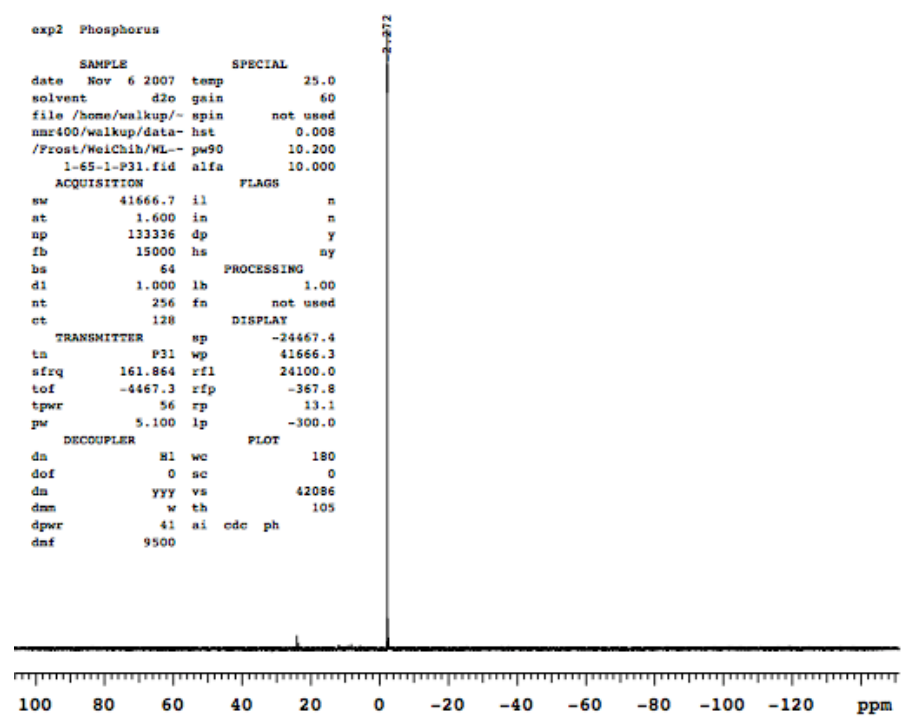

Synthesis of PTA- $\mathrm{CO}_{2} \mathrm{Li}$ and PTA- $\left(\mathrm{CO}_{2} \mathrm{Li}\right)_{2}$ without organic solvent. Carbon dioxide gas was carefully added along with a nitrogen stream to a $50 \mathrm{~mL}$ Schlenk flask charged with $2.03 \mathrm{~g}$ of PTA-Li $(12.7 \mathrm{mmol})$ at $-40{ }^{\circ} \mathrm{C}$. The off-white solid turned to a light yellow color upon exposure to $\mathrm{CO}_{2}$ gas. The sold bat was removed after 10 minutes and the system was stirred under a $\mathrm{CO}_{2}$ atmosphere overnight. The resulting yellow solid was washed with dichloromethane to remove residual PTA leaving behind the product PTA- $\mathrm{CO}_{2} \mathrm{Li}$ contaminated with 20\% PTA$\left(\mathrm{CO}_{2} \mathrm{Li}\right)_{2}$.

Synthesis of PTA $-{ }^{13} \mathrm{CO}_{2} \mathrm{Li}$ and PTA- $\left({ }^{13} \mathrm{CO}_{2} \mathrm{Li}\right)_{2}$. A Schlenk flask equipped with a magnetic stir bar was charged with $4.86 \mathrm{~g}(2.9 \mathrm{mmol})$ PTA-Li and placed in a liquid nitrogen bath. The system was placed under static vacuum and the atmosphere replaced by ${ }^{13} \mathrm{CO}_{2}$ from a $1 \mathrm{~L}$ breakneck flask. After stirring for two hours the cooling bath was removed and the reaction stirred at room temperature for an additional hour. The pale yellow product was washed with $4 \times 25 \mathrm{~mL}$ methylene chloride resulting in $3.63 \mathrm{~g}$ of the light yellow product (1.74 mmol, $60 \%$ isolated yield). ${ }^{31} \mathrm{P}\left\{{ }^{1} \mathrm{H}\right\}$ NMR $\left(162 \mathrm{MHz}, \mathrm{CD}_{3} \mathrm{OD}\right):-90.8 \mathrm{ppm}\left(\mathrm{d},{ }^{2} J_{\mathrm{PC}}=7.6 \mathrm{~Hz}, \mathrm{PTA}-{ }^{13} \mathrm{CO}_{2} \mathrm{Li}\right),-93.4 \mathrm{ppm}\left(\mathrm{t},{ }^{2} J_{\mathrm{PC}}=9.5 \mathrm{~Hz}\right.$, PTA$\left.\left({ }^{13} \mathrm{CO}_{2} \mathrm{Li}\right)_{2}\right) .{ }^{13} \mathrm{C}\left\{{ }^{1} \mathrm{H}\right\} \mathrm{NMR}\left(100 \mathrm{MHz}, \mathrm{CD}_{3} \mathrm{OD}\right): 175.5 \mathrm{ppm}\left(\mathrm{d},{ }^{2} J_{\mathrm{PC}}=7.4 \mathrm{~Hz}, \mathrm{PTA}^{-13} \mathrm{CO}_{2} \mathrm{Li}\right), 174.2 \mathrm{ppm}\left(\mathrm{d},{ }^{2} J_{\mathrm{PC}}=\right.$ $\left.9.7 \mathrm{~Hz}, \mathrm{PTA}-\left({ }^{13} \mathrm{CO}_{2} \mathrm{Li}\right)_{2}\right) .{ }^{1} \mathrm{H}$ NMR (400 MHz, $\left.\mathrm{CD}_{3} \mathrm{OD}\right): 4.91 \mathrm{ppm}(\mathrm{s}), 4.25-4.70 \mathrm{ppm}\left(\mathrm{m}, \mathrm{NCH}_{2} \mathrm{~N}\right), 4.65-4.10 \mathrm{ppm}$ (m, $\mathrm{PCH}_{2} \mathrm{~N} \& \mathrm{PCHN}$ ).

${ }^{31} \mathrm{P}\left\{{ }^{1} \mathrm{H}\right\}$ NMR $\left(162 \mathrm{MHz}, \mathrm{D}_{2} \mathrm{O}\right):-87.4 \mathrm{ppm}\left(\mathrm{t},{ }^{2} \mathrm{~J}_{\mathrm{P}}{ }^{13} \mathrm{C}=8.8 \mathrm{~Hz}\right.$, PTA- $\left.\left({ }^{13} \mathrm{CO}_{2} \mathrm{Li}\right)_{2}\right),-88.4 \mathrm{ppm}\left(\mathrm{d},{ }^{2} J_{\mathrm{P}}{ }^{13} \mathrm{C}=7.6 \mathrm{~Hz}\right.$, PTA$\left.{ }^{13} \mathrm{CO}_{2} \mathrm{Li}\right) .{ }^{13} \mathrm{C}\left\{{ }^{1} \mathrm{H}\right\}$ NMR $\left(100 \mathrm{MHz}, \mathrm{CD}_{3} \mathrm{OD}\right): 177.1 \mathrm{ppm}\left(\mathrm{d},{ }^{2} J_{\mathrm{P}}{ }^{13}{ }_{\mathrm{C}}=7.6 \mathrm{~Hz}, \mathrm{PTA}-{ }^{13} \mathrm{CO}_{2} \mathrm{Li}\right), 175.0 \mathrm{ppm}\left(\mathrm{d},{ }^{2} J_{\mathrm{PC}}=8.9\right.$ $\left.\mathrm{Hz}, \mathrm{PTA}-\left({ }^{13} \mathrm{CO}_{2} \mathrm{Li}\right)_{2}\right)$.

Scheme S-1. Proposed synthesis of PTA- $\left(\mathrm{CO}_{2} \mathrm{Li}\right)_{2}$.

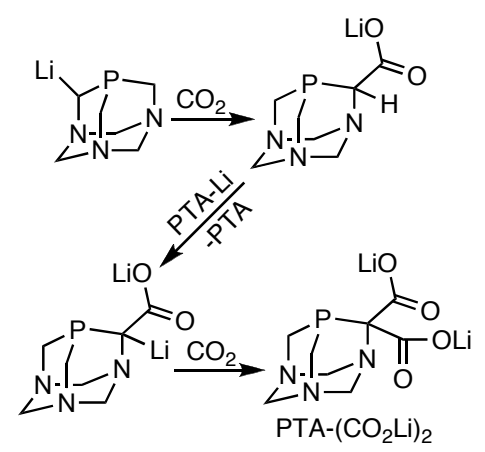


Figure 4-S. ESI mass spectra of PTA- $\mathrm{CO}_{2} \mathrm{Li}$ and $\mathrm{PTA}-{ }^{13} \mathrm{CO}_{2} \mathrm{Li}$.

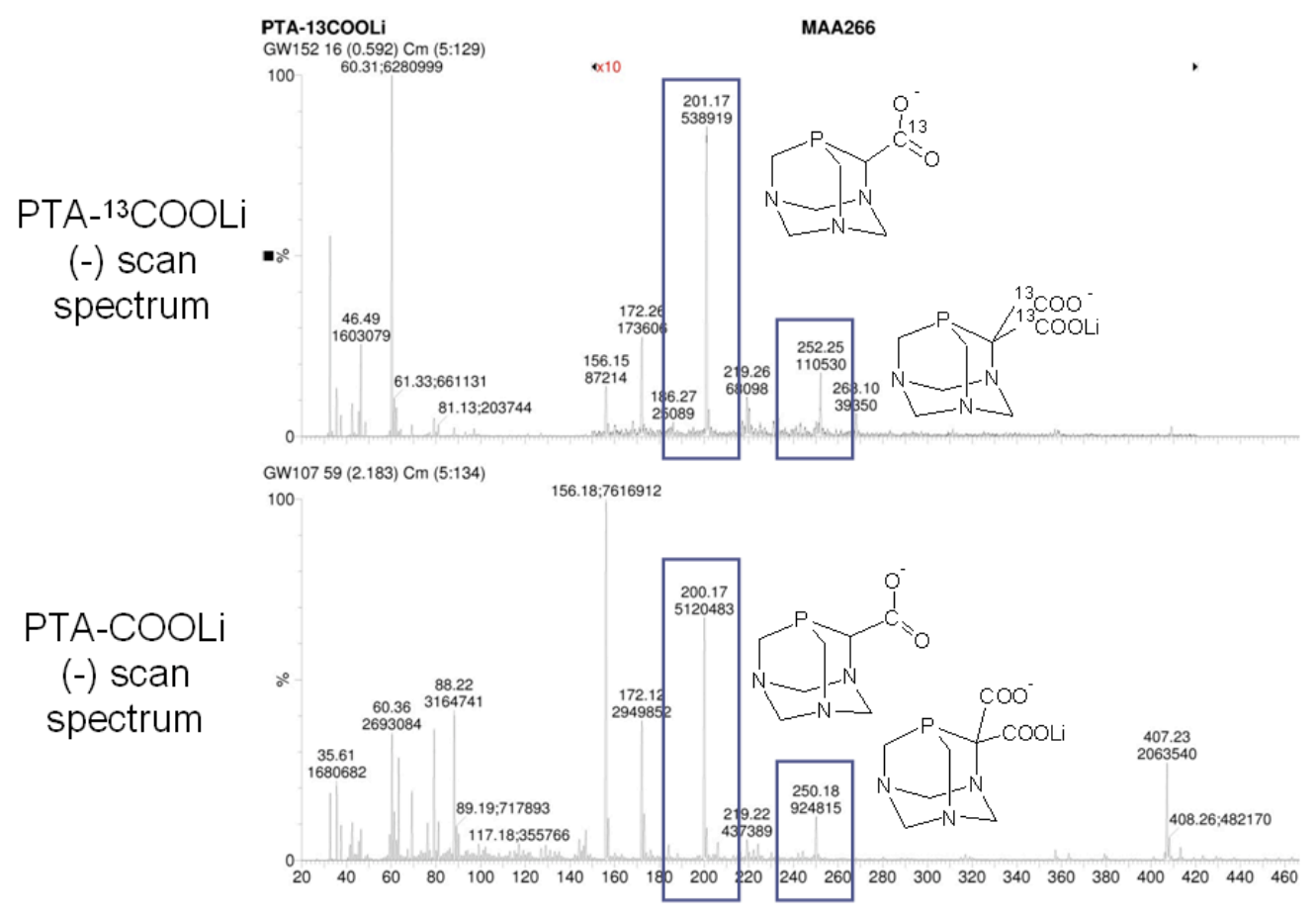

Figure 5-S. ${ }^{31} \mathrm{P}$ NMR spectrum of PTA- ${ }^{13} \mathrm{CO}_{2} \mathrm{Li}$ in $\mathrm{CD}_{3} \mathrm{OD}$.

STANDARD 1 H OBSERVE - profile
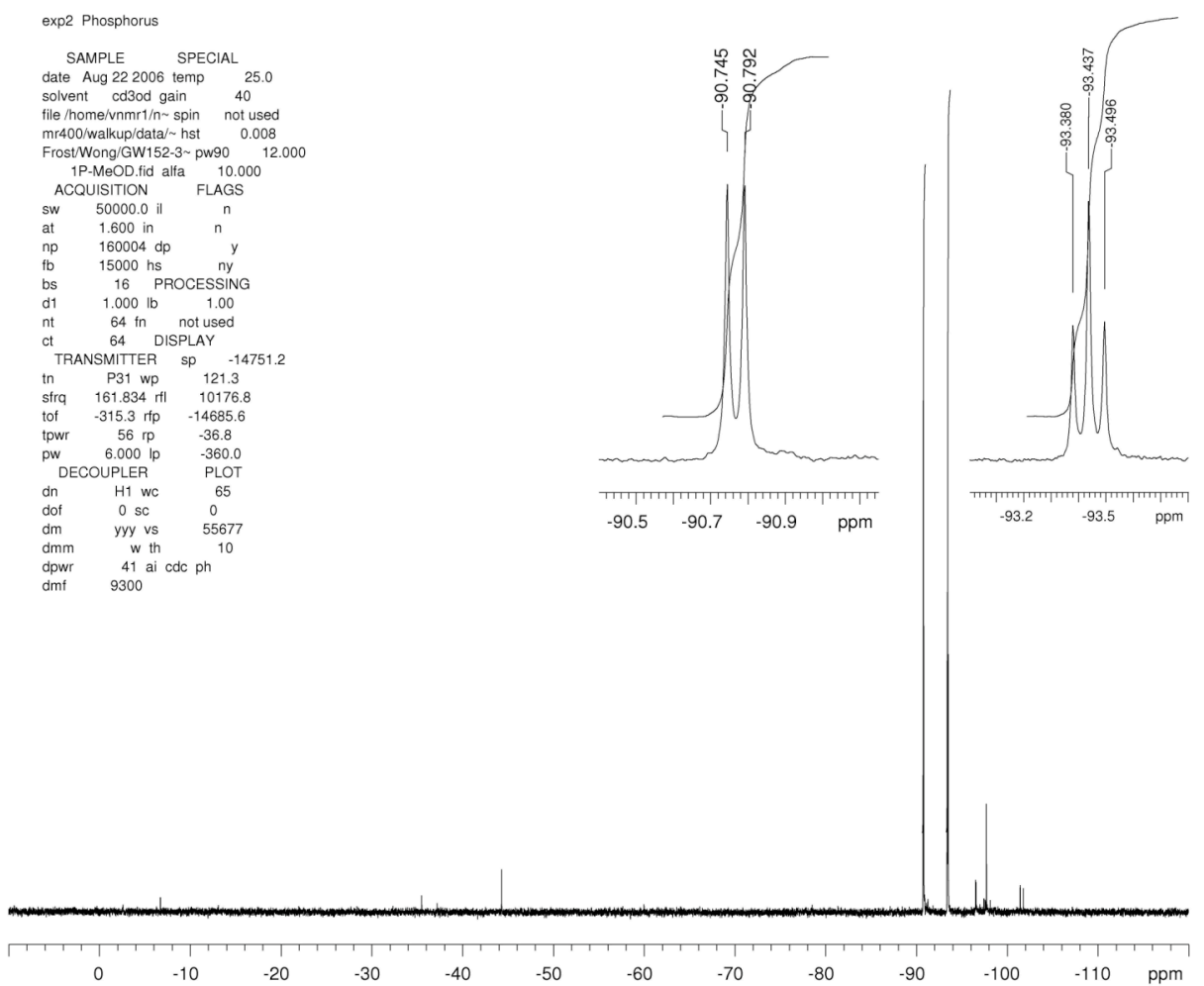
Figure 6-S. ${ }^{13} \mathrm{C}$ NMR spectrum of $\mathrm{PTA}^{-13} \mathrm{CO}_{2} \mathrm{Li}$ in $\mathrm{CD}_{3} \mathrm{OD}$.

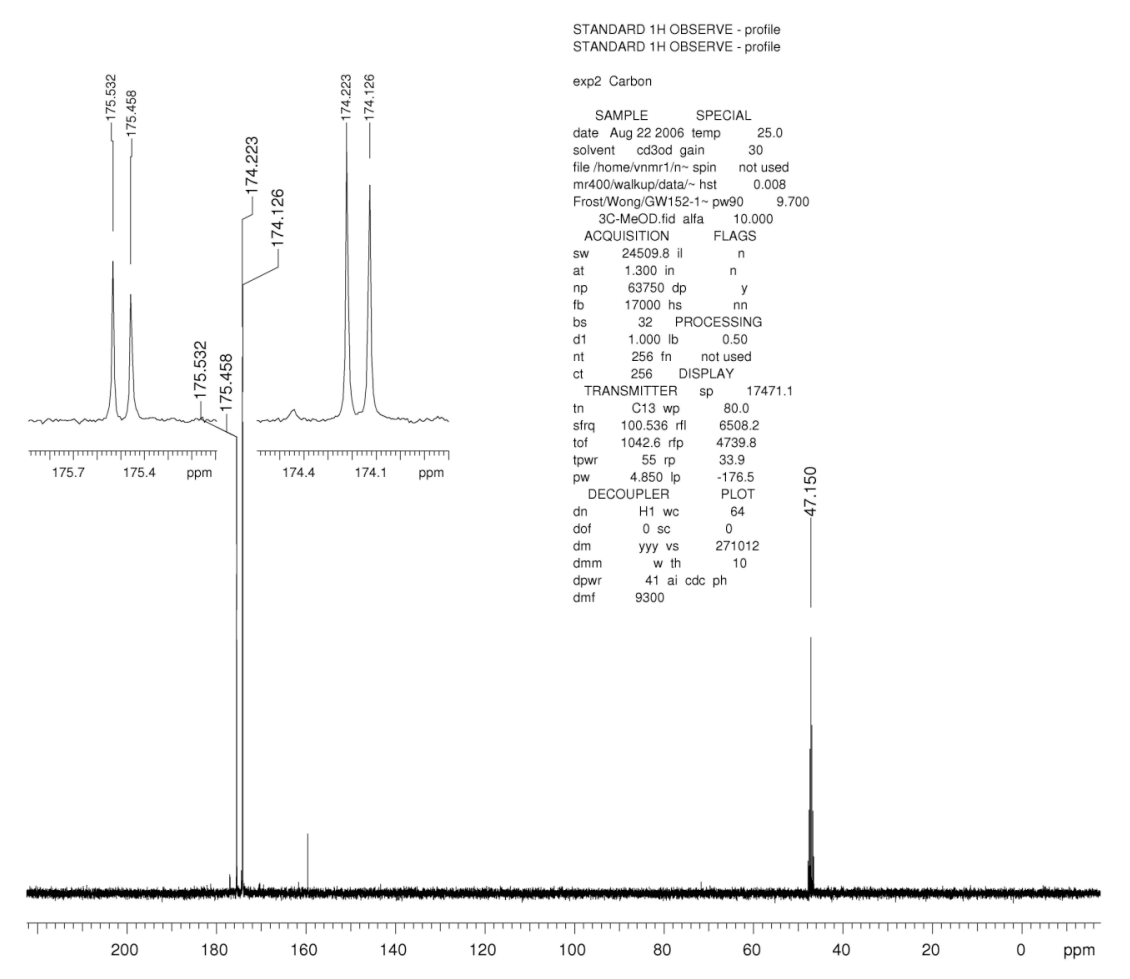

Figure 7-S. ${ }^{1} \mathrm{H}$ NMR spectrum of PTA- $\mathrm{CO}_{2} \mathrm{Me}$ in $\mathrm{CDCl}_{3}$

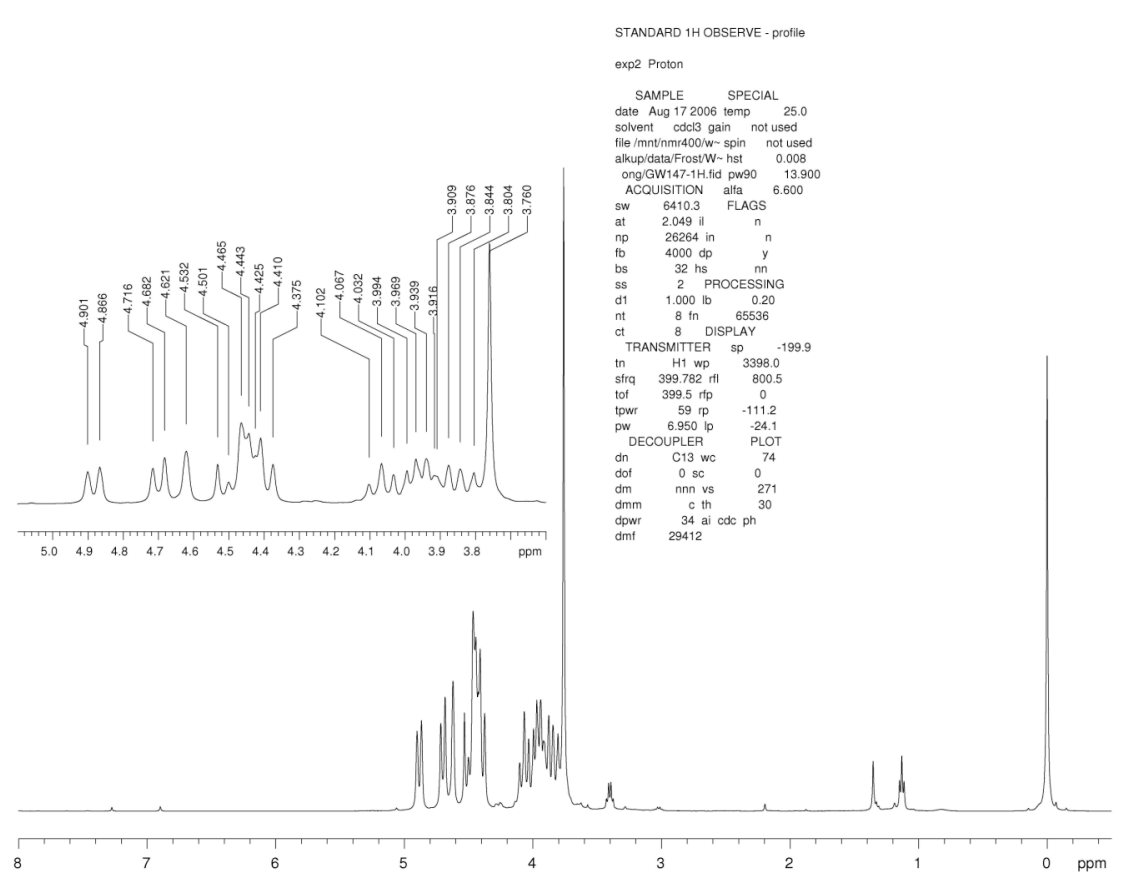


Figure 8-S. ${ }^{31} \mathrm{P}$ NMR spectrum of PTA- $\mathrm{CO}_{2} \mathrm{Me}$ in $\mathrm{CDCl}_{3}$.

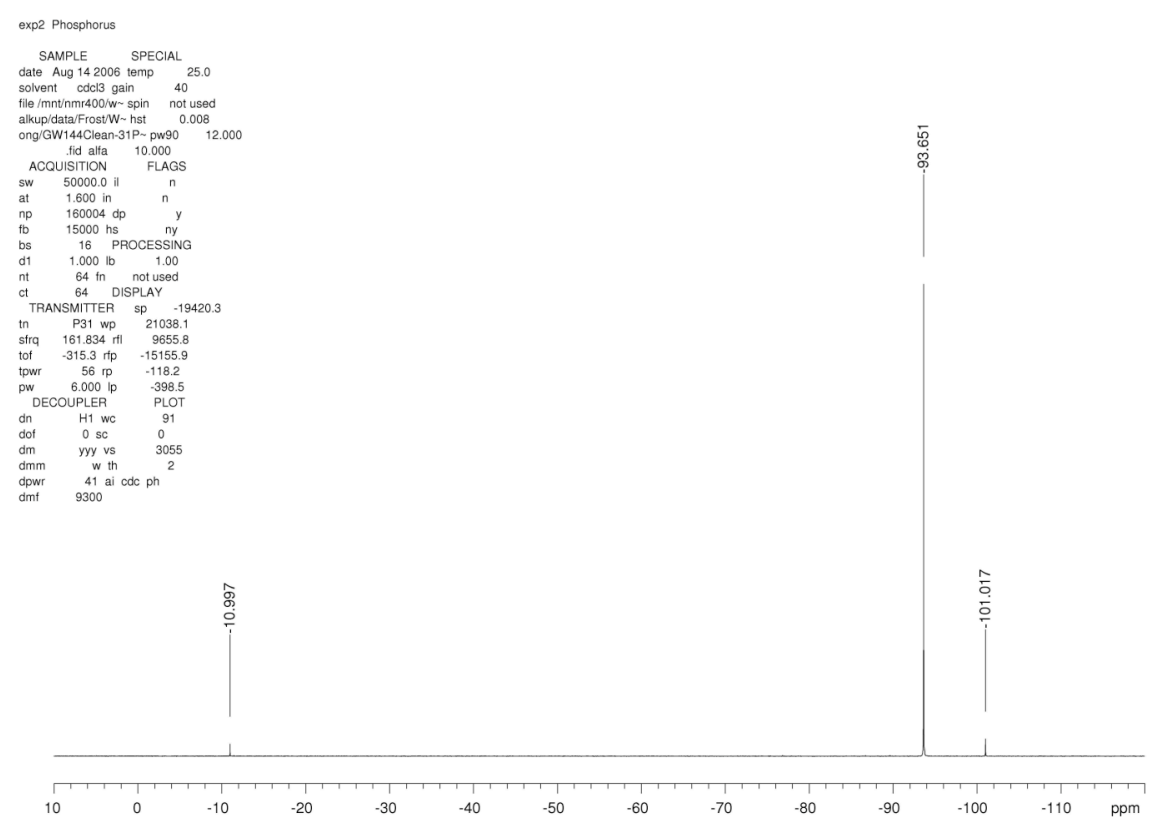

Figure 9-S. ${ }^{31} \mathrm{P}$ NMR spectrum of $\mathrm{O}=\mathrm{PTA}-\mathrm{CO}_{2} \mathrm{Me}$ in $\mathrm{D}_{2} \mathrm{O}$.

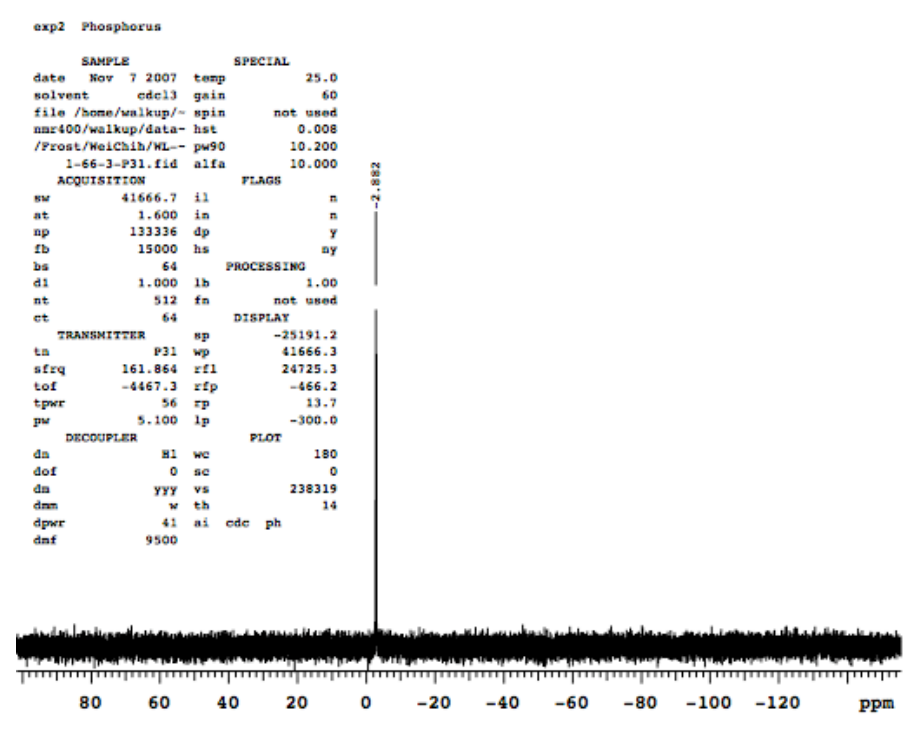


Figure 10-S. ${ }^{1} \mathrm{H}$ NMR spectrum of PTA-C $\left(\mathrm{C}_{6} \mathrm{H}_{5}\right)_{2} \mathrm{OH}$ in $\mathrm{CDCl}_{3}$.

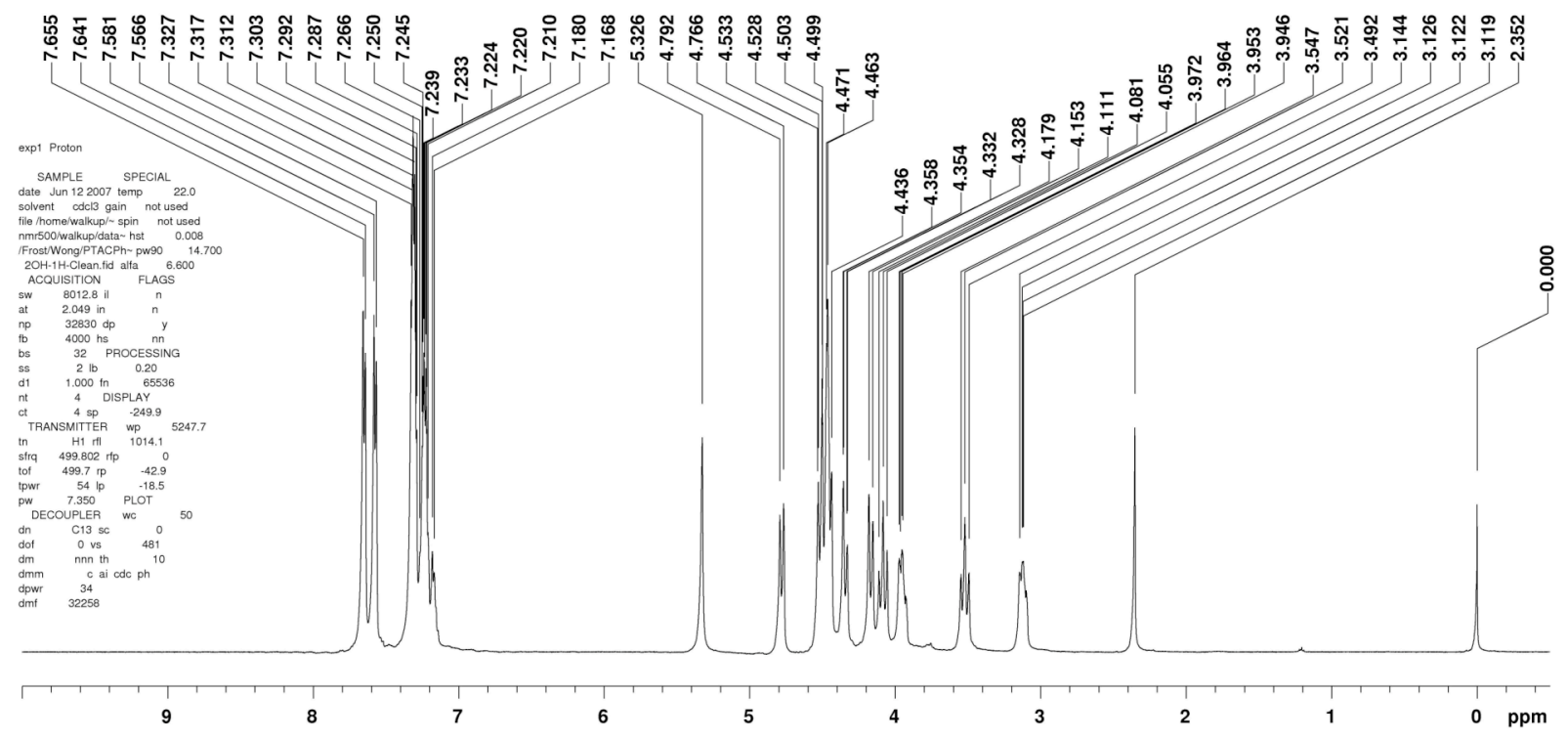

Figure 11-S. ${ }^{31} \mathrm{P}$ NMR spectrum of PTA-C $\left(\mathrm{C}_{6} \mathrm{H}_{5}\right)_{2} \mathrm{OH}$ in $\mathrm{CDCl}_{3}$.

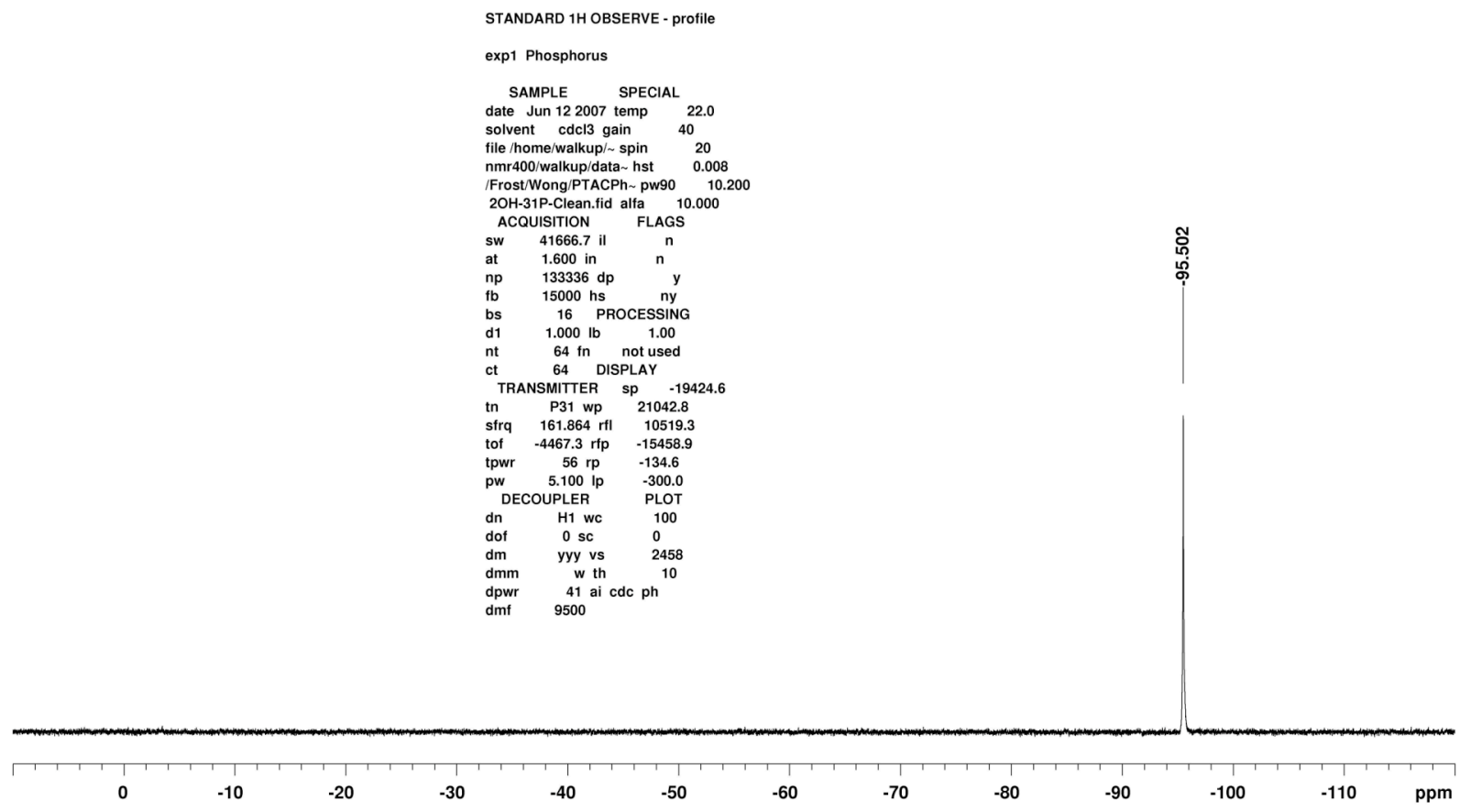


Figure 12-S. ${ }^{31} \mathrm{P}\left\{{ }^{1} \mathrm{H}\right\}$ NMR spectrum of $\mathrm{O}=\mathrm{PTA}-\mathrm{C}\left(\mathrm{C}_{6} \mathrm{H}_{5}\right)_{2} \mathrm{OH}$ in $\mathrm{D}_{2} \mathrm{O}$.

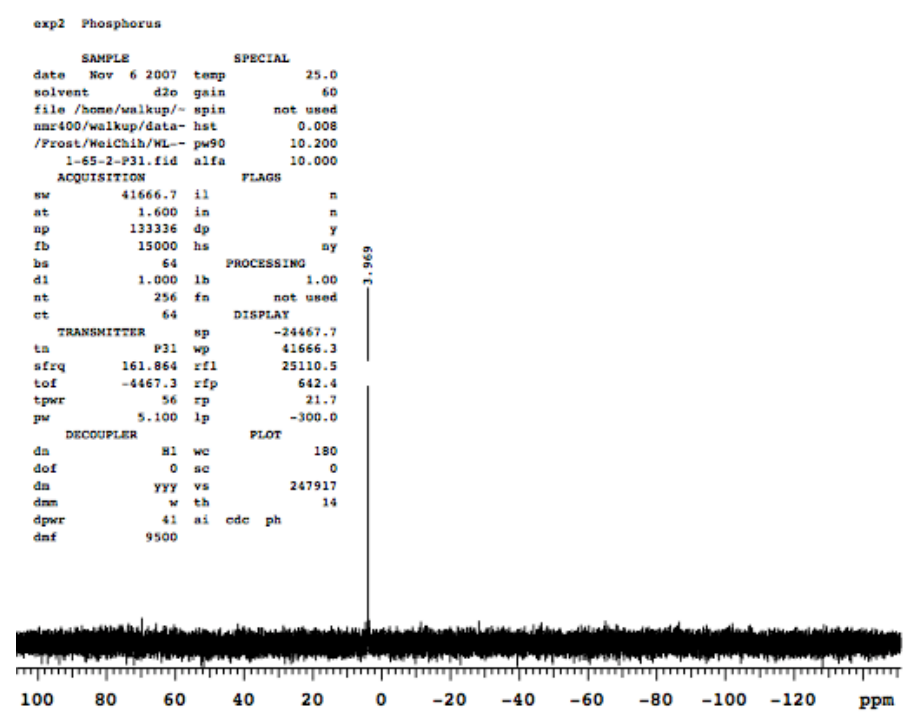

Figure 13-S. ${ }^{1} \mathrm{H}$ NMR spectrum of PTA- $\mathrm{C}_{6}\left(\mathrm{C}_{6} \mathrm{H}_{4} \mathrm{OCH}_{3}\right)_{2} \mathrm{OH}$ in $\mathrm{CDCl}_{3}$.

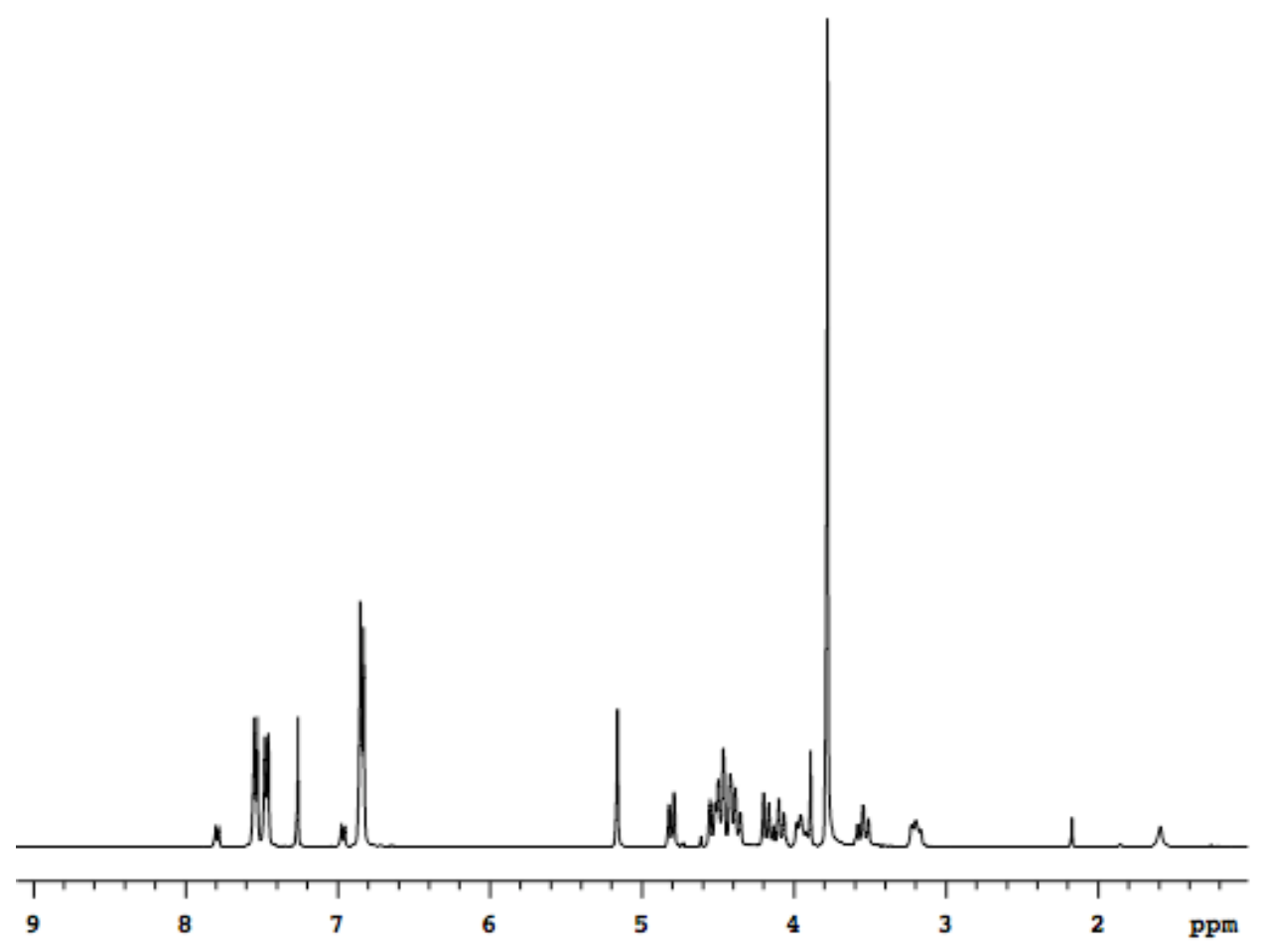


Figure 14-S. ${ }^{31} \mathrm{P}\left\{{ }^{1} \mathrm{H}\right\}$ NMR spectrum of PTA- $\mathrm{C}\left(\mathrm{C}_{6} \mathrm{H}_{4} \mathrm{OCH}_{3}\right)_{2} \mathrm{OH}$ in $\mathrm{CDCl}_{3}$.
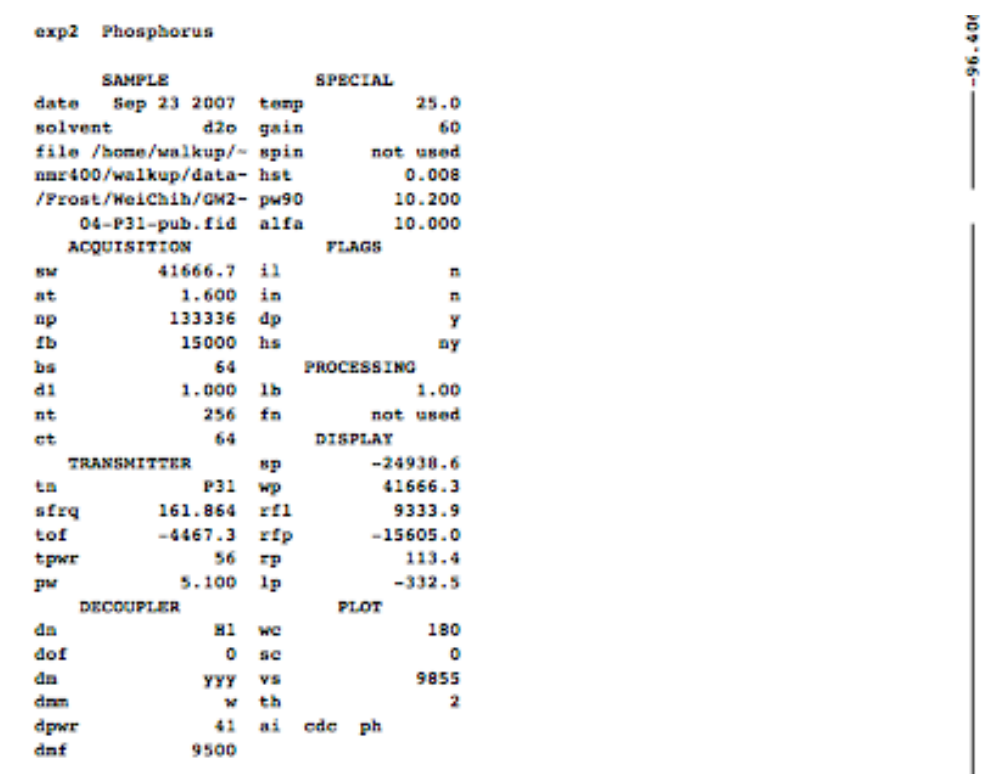

ग

$\begin{array}{llllllllllll}80 & 60 & 40 & 20 & 0 & -20 & -40 & -60 & -80 & -100 & -120 & \text { ppm }\end{array}$

Figure 15-S. ${ }^{31} \mathrm{P}\left\{{ }^{1} \mathrm{H}\right\}$ NMR spectrum of $\mathrm{O}=\mathrm{PTA}-\mathrm{C}\left(\mathrm{C}_{6} \mathrm{H}_{4} \mathrm{OCH}\right)_{3} \mathrm{OH}$ in $\mathrm{D}_{2} \mathrm{O}$.

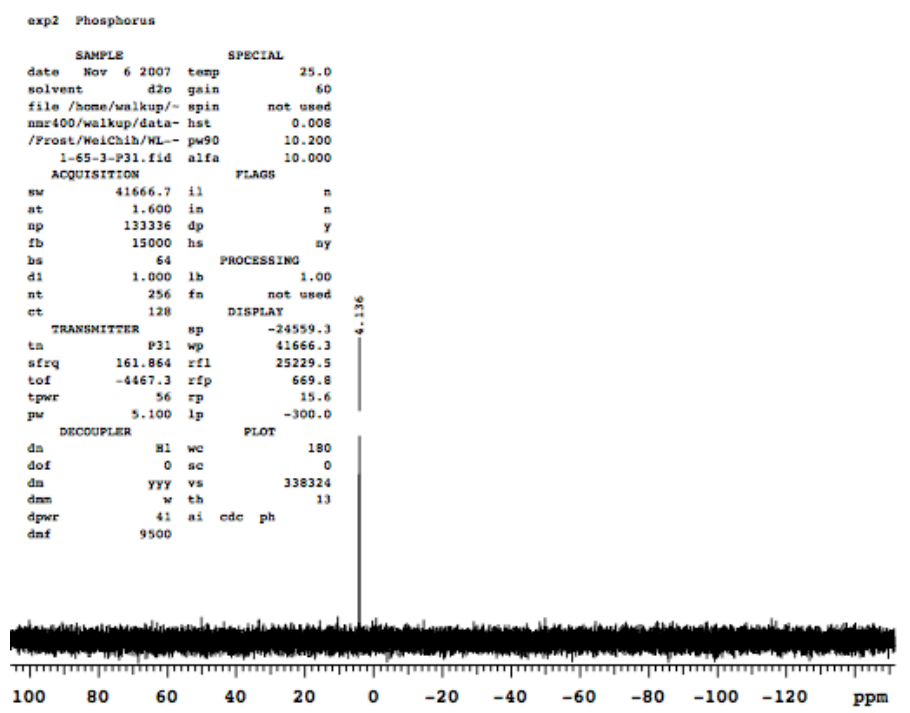


Figure 16-S. ${ }^{1} \mathrm{H}$ NMR spectrum of PTA- $\mathrm{CH}\left(\mathrm{C}_{6} \mathrm{H}_{4} \mathrm{OCH}_{3}\right) \mathrm{OH}$ in $\mathrm{CDCl}_{3}$.

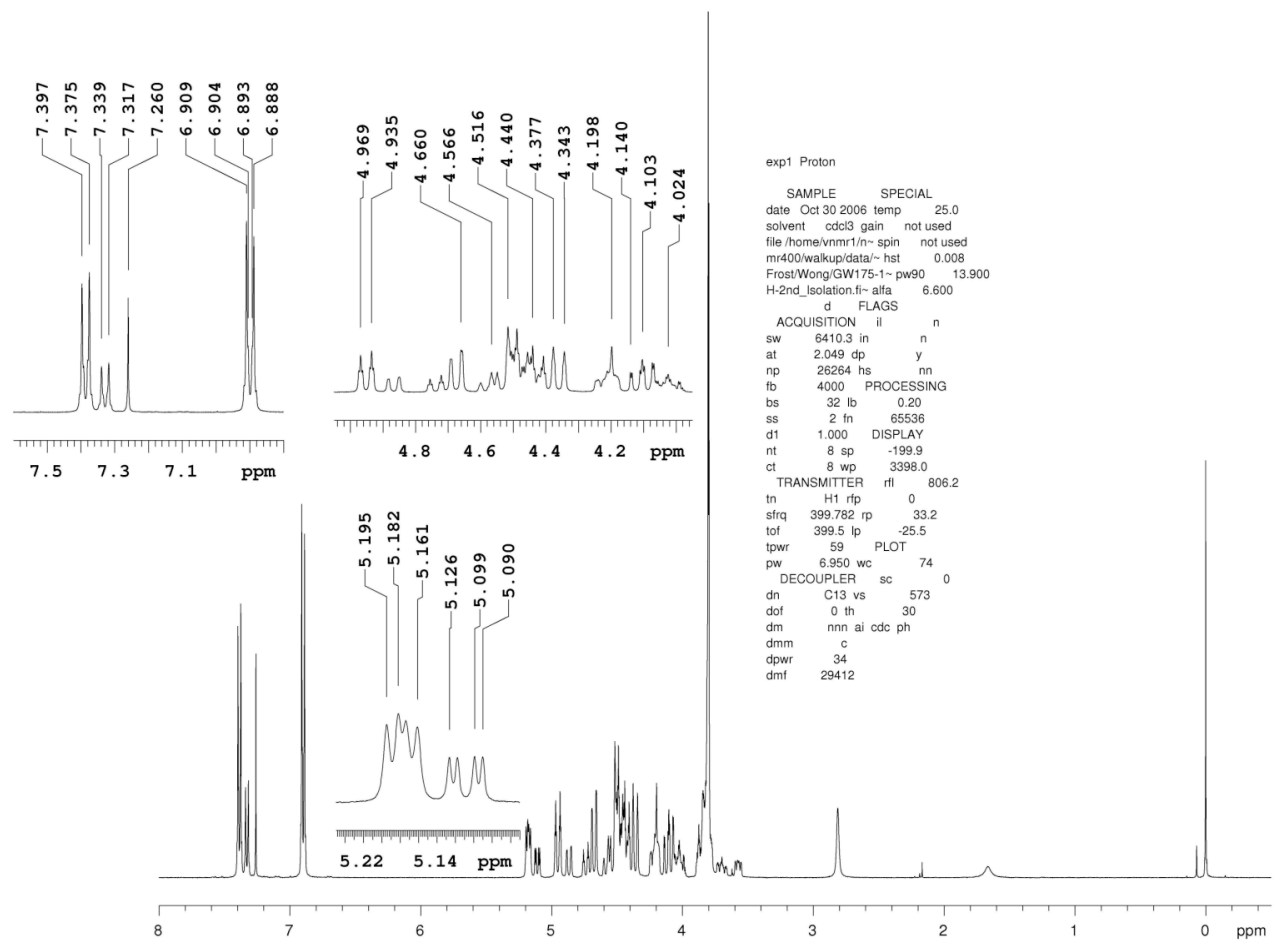

Figure 17-S. ${ }^{31} \mathrm{P}\left\{{ }^{1} \mathrm{H}\right\}$ NMR spectrum of PTA- $\mathrm{CH}\left(\mathrm{C}_{6} \mathrm{H}_{4} \mathrm{OCH}_{3}\right) \mathrm{OH}$ in $\mathrm{CDCl}_{3}$.
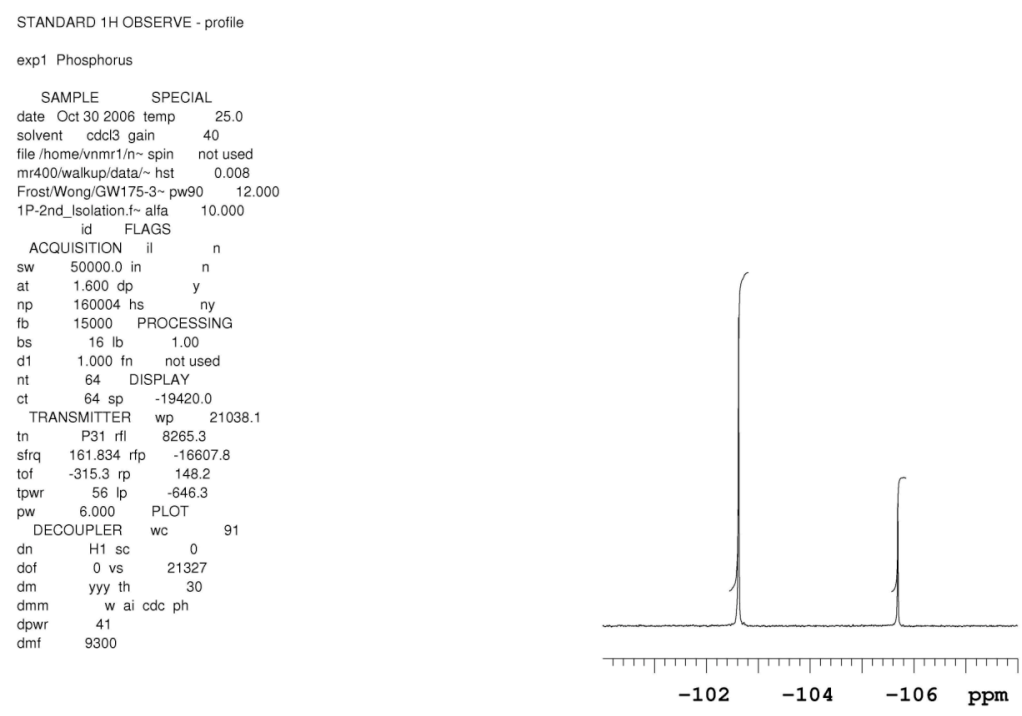
Figure 18-S. ${ }^{31} \mathrm{P}\left\{{ }^{1} \mathrm{H}\right\}$ NMR spectrum of $\mathrm{O}=\mathrm{PTA}-\mathrm{CH}\left(\mathrm{C}_{6} \mathrm{H}_{4} \mathrm{OCH}_{3}\right) \mathrm{OH}$ in $\mathrm{D}_{2} \mathrm{O}$.

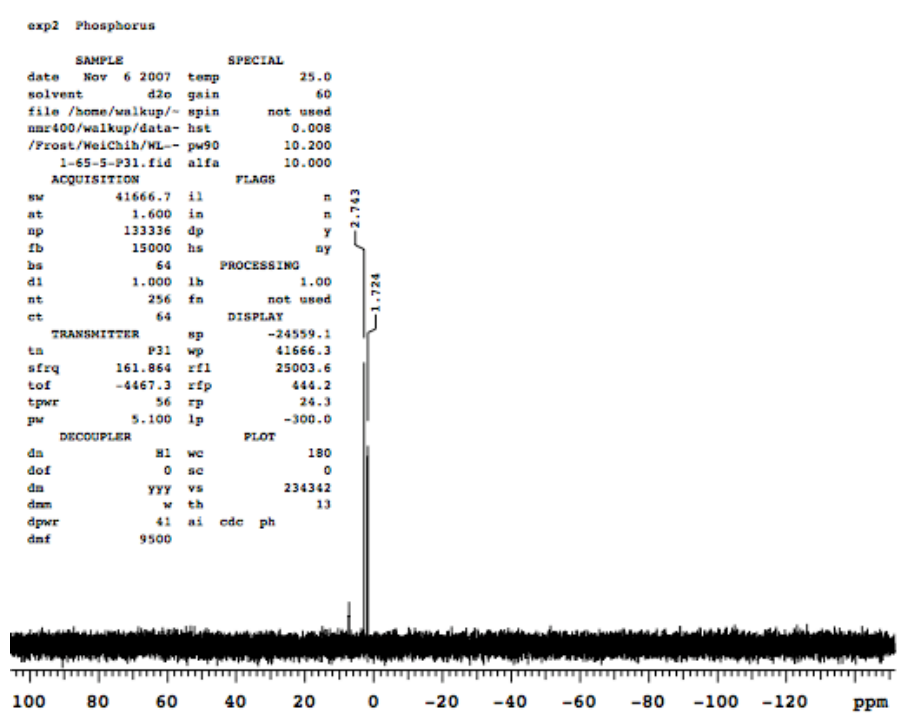

Figure 19-S. ${ }^{1} \mathrm{H}$ NMR spectrum of PTA-CH(ferrocenyl)OH in $\mathrm{CDCl}_{3}$.

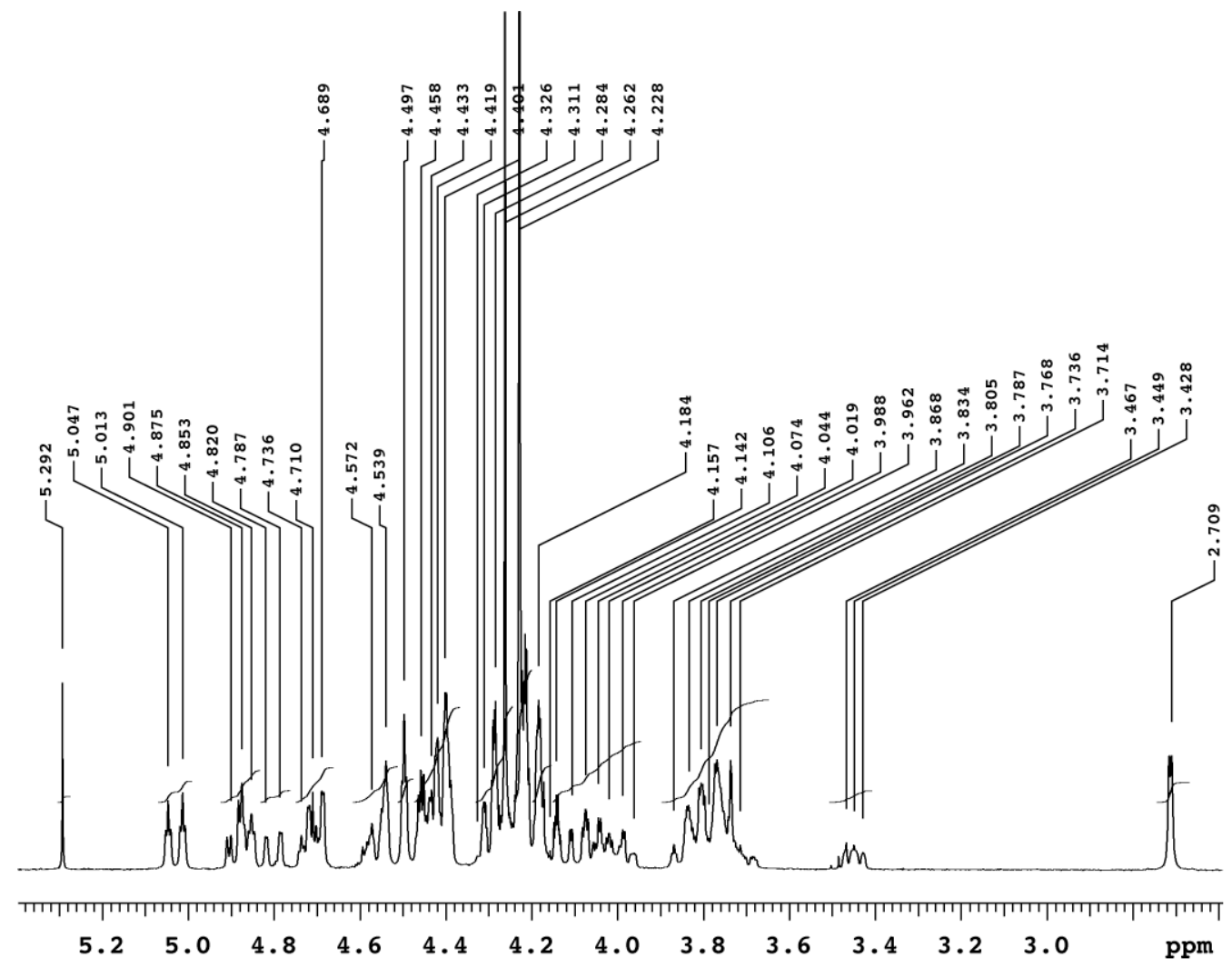


Figure $20-\mathrm{S} .{ }^{31} \mathrm{P}\left\{{ }^{1} \mathrm{H}\right\}$ NMR spectrum of PTA-CH(ferrocenyl)OH in $\mathrm{CDCl}_{3}$.
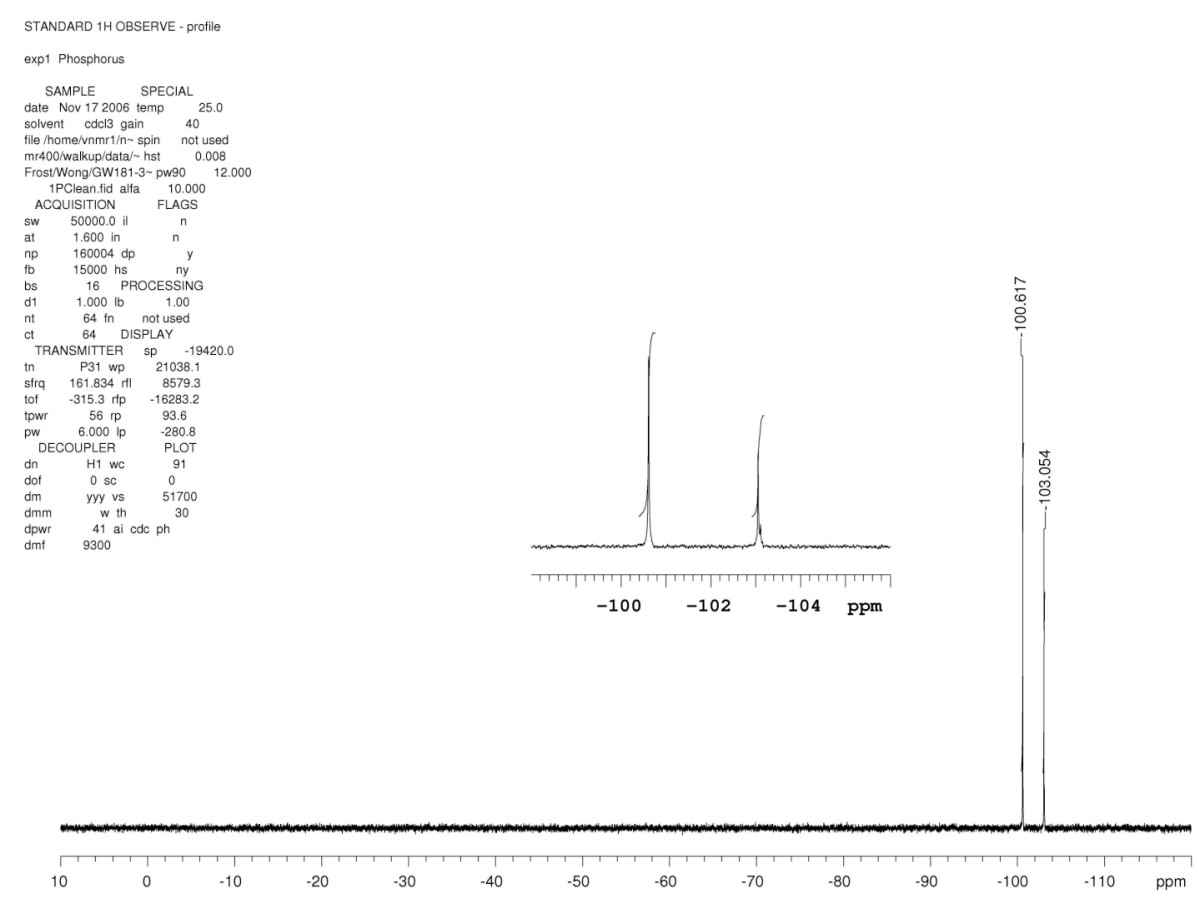

Figure 21-S. ${ }^{31} \mathrm{P}\left\{{ }^{1} \mathrm{H}\right\}$ NMR spectrum of $\mathrm{O}=\mathrm{PTA}-\mathrm{CH}$ (ferrocenyl)OH in $\mathrm{D}_{2} \mathrm{O}$.

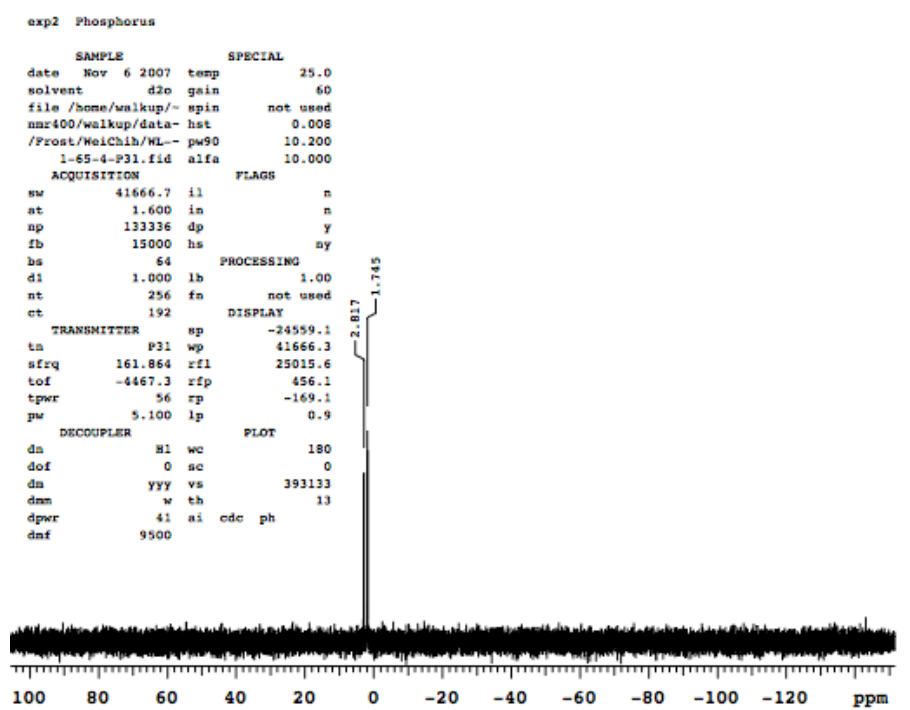


Figure 22-S. ${ }^{1} \mathrm{H}$ NMR spectrum of $\left(\eta^{6}-\mathrm{C}_{6} \mathrm{H}_{5} \mathrm{CH}_{3}\right) \mathrm{RuCl}_{2}\left(\mathrm{PTA}-\mathrm{C}\left(\mathrm{C}_{6} \mathrm{H}_{5}\right)_{2} \mathrm{OH}\right)$ in $\mathrm{CDCl}_{3}$.

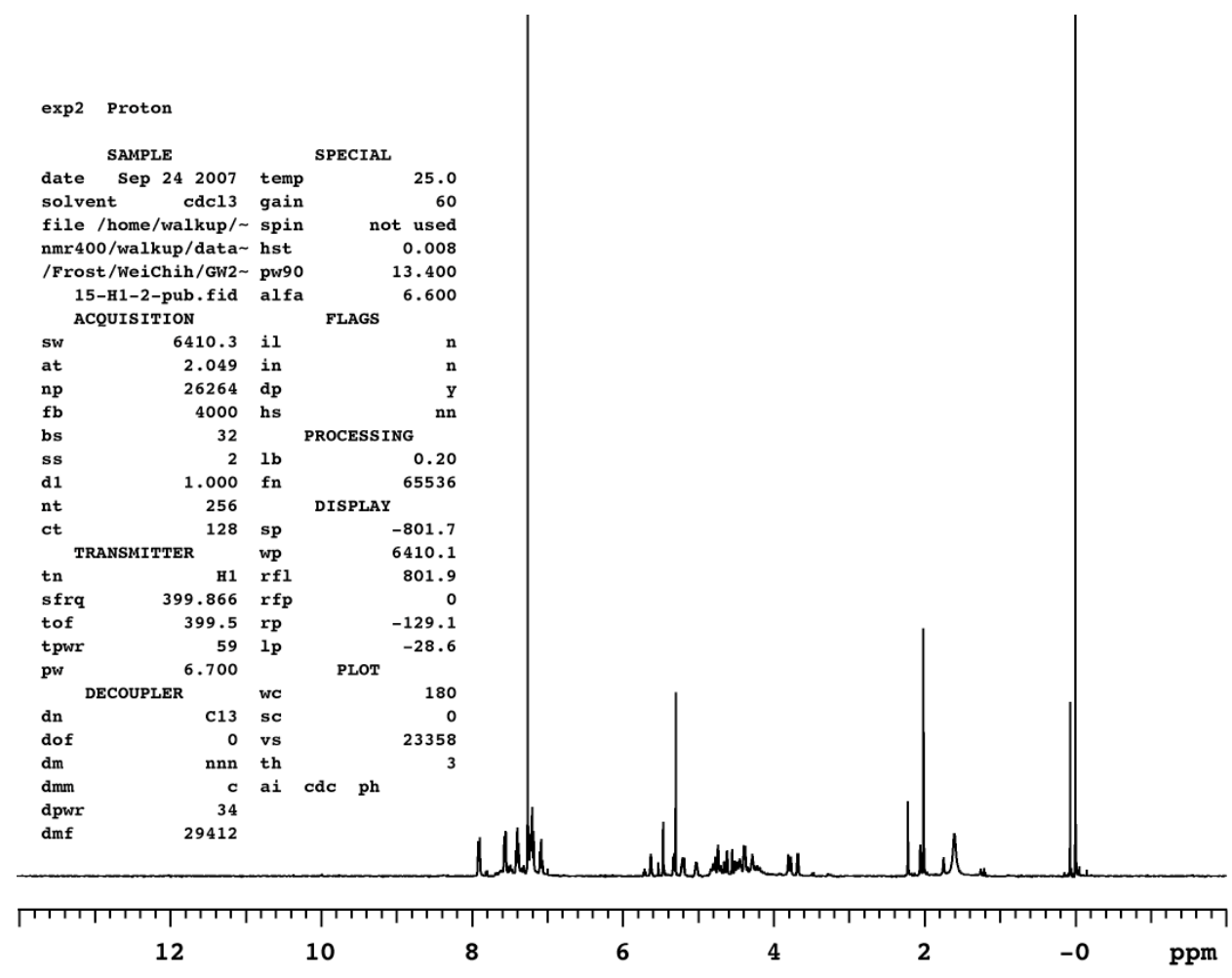

Figure 23-S. ${ }^{31} \mathrm{P}\left\{{ }^{1} \mathrm{H}\right\}$ NMR spectrum of $\left(\eta^{6}-\mathrm{C}_{6} \mathrm{H}_{5} \mathrm{CH}_{3}\right) \mathrm{RuCl}_{2}\left(\mathrm{PTA}-\mathrm{C}\left(\mathrm{C}_{6} \mathrm{H}_{5}\right)_{2} \mathrm{OH}\right)$ in $\mathrm{CDCl}_{3}$.
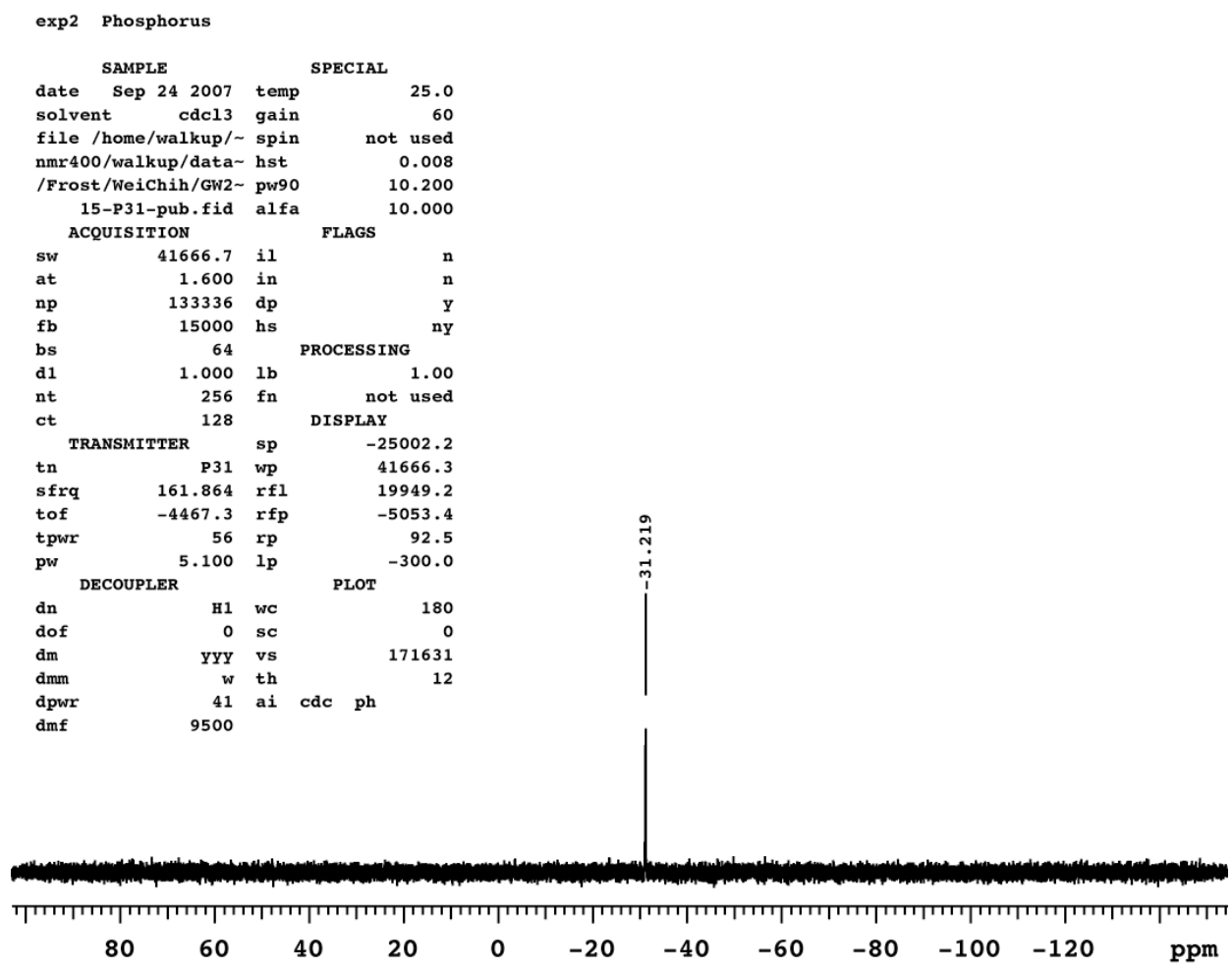
Figure 24-S. ${ }^{1} \mathrm{H}$ NMR spectrum of $\left(\eta^{6}-\mathrm{C}_{6} \mathrm{H}_{6}\right) \mathrm{RuCl}_{2}\left(\mathrm{PTA}-\mathrm{C}\left(\mathrm{C}_{6} \mathrm{H}_{4} \mathrm{OCH}_{3}\right)_{2} \mathrm{OH}\right)$ in $\mathrm{CDCl}_{3}$.

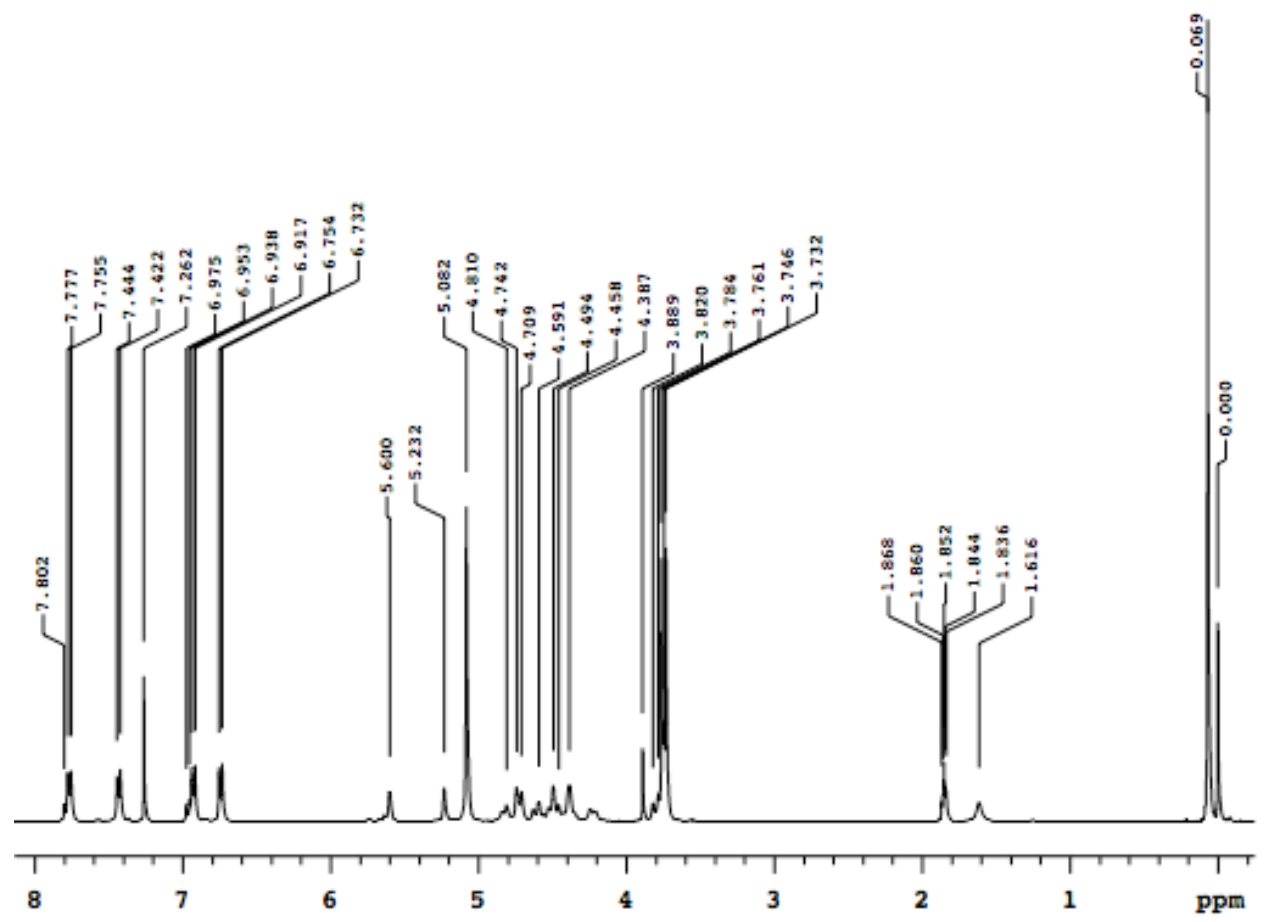

Figure 25-S. ${ }^{31} \mathrm{P}\left\{{ }^{1} \mathrm{H}\right\}$ NMR spectrum of $\left(\eta^{6}-\mathrm{C}_{6} \mathrm{H}_{6}\right) \mathrm{RuCl}_{2}\left(\mathrm{PTA}-\mathrm{C}\left(\mathrm{C}_{6} \mathrm{H}_{4} \mathrm{OCH}_{3}\right)_{2} \mathrm{OH}\right)$ in $\mathrm{CDCl}_{3}$.

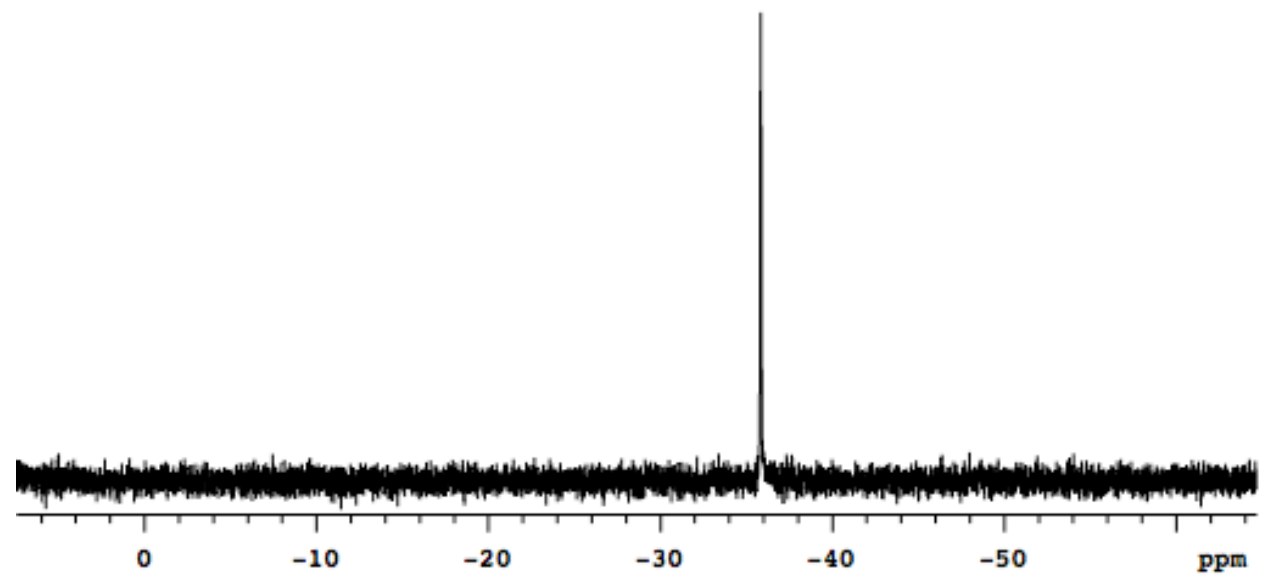


Figure 26-S. ${ }^{31} \mathrm{P}\left\{{ }^{1} \mathrm{H}\right\}$ NMR spectrum of $\left(\eta^{6}-\mathrm{C}_{6} \mathrm{H}_{6}\right) \mathrm{RuCl}_{2}\left(\mathrm{PTA}-\mathrm{CH}\left(\mathrm{C}_{6} \mathrm{H}_{4} \mathrm{OCH}_{3}\right) \mathrm{OH}\right)$ in $\mathrm{CDCl}_{3}$.

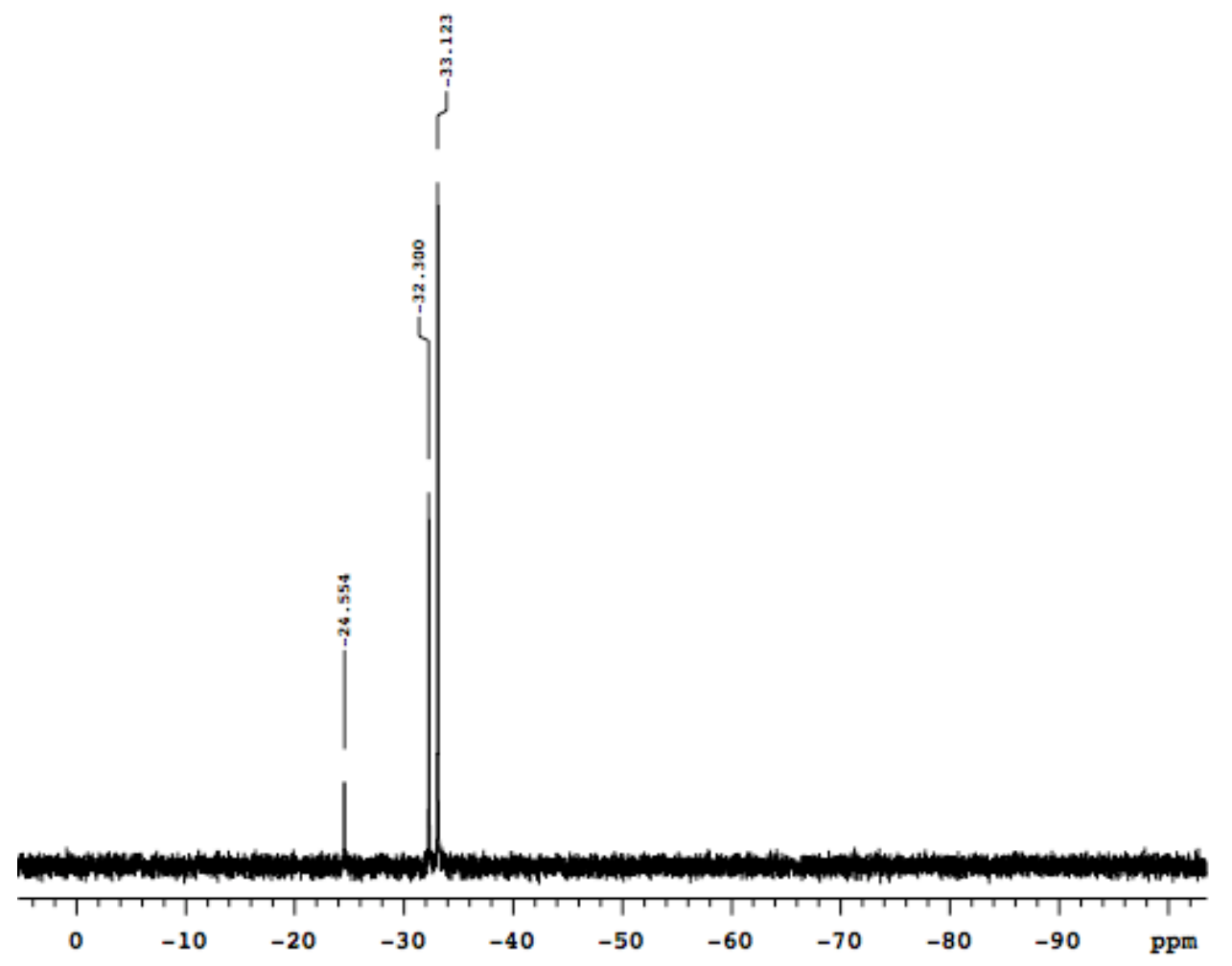

Figure 27-S. Ball and stick representation of a $\kappa^{1}(\mathrm{P})-\kappa^{2}(\mathrm{~N}, \mathrm{O})$ binding mode of [PTA-CH(ferrocenyl)O-]. Crystals were obtained from the reaction of PTA- $\mathrm{CH}\left(\right.$ ferrocenyl)OH with $\left[\left(\eta^{6}-\mathrm{C}_{6} \mathrm{H}_{6}\right) \mathrm{RuCl}_{2}\right]_{2}$ in $\mathrm{CH}_{2} \mathrm{Cl}_{2}$.

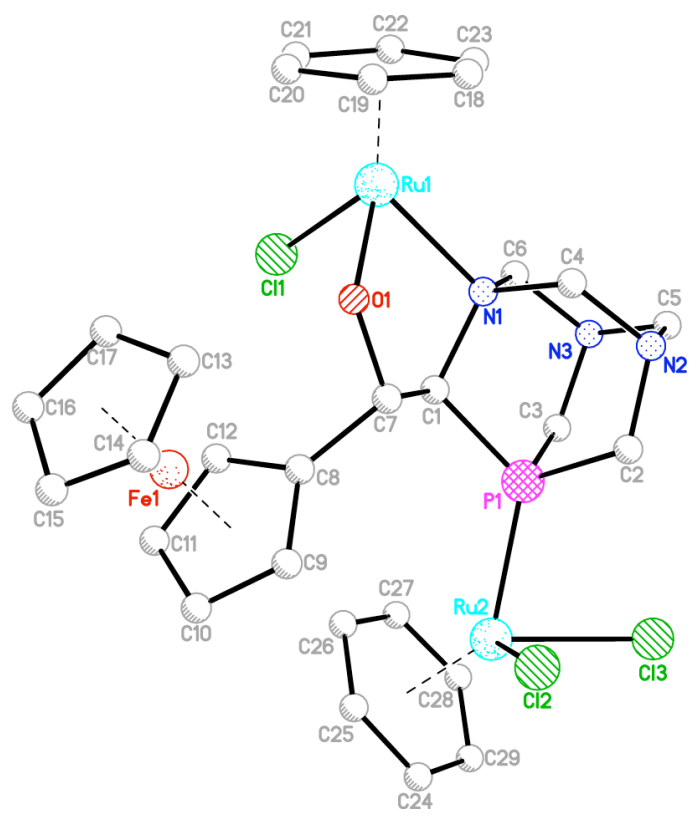


Table 1-S. Bond lengths $[\AA]$ and angles $\left[{ }^{\circ}\right]$ for $\mathrm{O}=\mathrm{PTA}-\mathrm{CO}_{2} \mathrm{Me}$.

\begin{tabular}{|c|c|c|c|}
\hline $\mathrm{P}(1)-\mathrm{O}(3)$ & $1.483(3)$ & $\mathrm{C}(5)-\mathrm{N}(3)-\mathrm{C}(3)$ & $111.1(3)$ \\
\hline $\mathrm{P}(1)-\mathrm{C}(2)$ & $1.815(4)$ & $\mathrm{N}(1)-\mathrm{C}(1)-\mathrm{C}(7)$ & $116.6(3)$ \\
\hline $\mathrm{P}(1)-\mathrm{C}(3)$ & $1.818(4)$ & $\mathrm{N}(1)-\mathrm{C}(1)-\mathrm{P}(1)$ & $109.9(2)$ \\
\hline $\mathrm{P}(1)-\mathrm{C}(1)$ & $1.834(4)$ & $\mathrm{C}(7)-\mathrm{C}(1)-\mathrm{P}(1)$ & $110.2(3)$ \\
\hline $\mathrm{O}(1)-\mathrm{C}(7)$ & $1.219(5)$ & $\mathrm{N}(1)-\mathrm{C}(1)-\mathrm{H}(1 \mathrm{~A})$ & 106.5 \\
\hline $\mathrm{O}(2)-\mathrm{C}(7)$ & $1.324(5)$ & $\mathrm{C}(7)-\mathrm{C}(1)-\mathrm{H}(1 \mathrm{~A})$ & 106.5 \\
\hline $\mathrm{O}(2)-\mathrm{C}(8)$ & $1.450(5)$ & $\mathrm{P}(1)-\mathrm{C}(1)-\mathrm{H}(1 \mathrm{~A})$ & 106.5 \\
\hline$N(1)-C(4)$ & $1.477(4)$ & $\mathrm{N}(2)-\mathrm{C}(2)-\mathrm{P}(1)$ & $110.7(2)$ \\
\hline$N(1)-C(6)$ & $1.479(5)$ & $\mathrm{N}(2)-\mathrm{C}(2)-\mathrm{H}(2 \mathrm{~A})$ & 109.5 \\
\hline $\mathrm{N}(1)-\mathrm{C}(1)$ & $1.484(5)$ & $\mathrm{P}(1)-\mathrm{C}(2)-\mathrm{H}(2 \mathrm{~A})$ & 109.5 \\
\hline$N(2)-C(5)$ & $1.467(5)$ & $\mathrm{N}(2)-\mathrm{C}(2)-\mathrm{H}(2 \mathrm{~B})$ & 109.5 \\
\hline$N(2)-C(4)$ & $1.469(4)$ & $\mathrm{P}(1)-\mathrm{C}(2)-\mathrm{H}(2 \mathrm{~B})$ & 109.5 \\
\hline$N(2)-C(2)$ & $1.474(5)$ & $\mathrm{H}(2 \mathrm{~A})-\mathrm{C}(2)-\mathrm{H}(2 \mathrm{~B})$ & 108.1 \\
\hline$N(3)-C(6)$ & $1.459(4)$ & $\mathrm{N}(3)-\mathrm{C}(3)-\mathrm{P}(1)$ & $110.3(2)$ \\
\hline$N(3)-C(5)$ & $1.472(4)$ & $\mathrm{N}(3)-\mathrm{C}(3)-\mathrm{H}(3 \mathrm{~B})$ & 109.6 \\
\hline$N(3)-C(3)$ & $1.480(5)$ & $\mathrm{P}(1)-\mathrm{C}(3)-\mathrm{H}(3 \mathrm{~B})$ & 109.6 \\
\hline $\mathrm{C}(1)-\mathrm{C}(7)$ & $1.491(5)$ & $\mathrm{N}(3)-\mathrm{C}(3)-\mathrm{H}(3 \mathrm{C})$ & 109.6 \\
\hline $\mathrm{C}(1)-\mathrm{H}(1 \mathrm{~A})$ & 1.0000 & $\mathrm{P}(1)-\mathrm{C}(3)-\mathrm{H}(3 \mathrm{C})$ & 109.6 \\
\hline $\mathrm{C}(2)-\mathrm{H}(2 \mathrm{~A})$ & 0.9900 & $\mathrm{H}(3 \mathrm{~B})-\mathrm{C}(3)-\mathrm{H}(3 \mathrm{C})$ & 108.1 \\
\hline $\mathrm{C}(2)-\mathrm{H}(2 \mathrm{~B})$ & 0.9900 & $\mathrm{~N}(2)-\mathrm{C}(4)-\mathrm{N}(1)$ & $114.4(3)$ \\
\hline $\mathrm{C}(3)-\mathrm{H}(3 \mathrm{~B})$ & 0.9900 & $\mathrm{~N}(2)-\mathrm{C}(4)-\mathrm{H}(4 \mathrm{~A})$ & 108.7 \\
\hline $\mathrm{C}(3)-\mathrm{H}(3 \mathrm{C})$ & 0.9900 & $\mathrm{~N}(1)-\mathrm{C}(4)-\mathrm{H}(4 \mathrm{~A})$ & 108.7 \\
\hline $\mathrm{C}(4)-\mathrm{H}(4 \mathrm{~A})$ & 0.9900 & $\mathrm{~N}(2)-\mathrm{C}(4)-\mathrm{H}(4 \mathrm{~B})$ & 108.7 \\
\hline $\mathrm{C}(4)-\mathrm{H}(4 \mathrm{~B})$ & 0.9900 & $\mathrm{~N}(1)-\mathrm{C}(4)-\mathrm{H}(4 \mathrm{~B})$ & 108.7 \\
\hline $\mathrm{C}(5)-\mathrm{H}(5 \mathrm{~A})$ & 0.9900 & $\mathrm{H}(4 \mathrm{~A})-\mathrm{C}(4)-\mathrm{H}(4 \mathrm{~B})$ & 107.6 \\
\hline $\mathrm{C}(5)-\mathrm{H}(5 \mathrm{~B})$ & 0.9900 & $\mathrm{~N}(2)-\mathrm{C}(5)-\mathrm{N}(3)$ & $114.4(3)$ \\
\hline $\mathrm{C}(6)-\mathrm{H}(6 \mathrm{~A})$ & 0.9900 & $\mathrm{~N}(2)-\mathrm{C}(5)-\mathrm{H}(5 \mathrm{~A})$ & 108.7 \\
\hline $\mathrm{C}(6)-\mathrm{H}(6 \mathrm{~B})$ & 0.9900 & $\mathrm{~N}(3)-\mathrm{C}(5)-\mathrm{H}(5 \mathrm{~A})$ & 108.7 \\
\hline $\mathrm{C}(8)-\mathrm{H}(8 \mathrm{~A})$ & 0.9800 & $\mathrm{~N}(2)-\mathrm{C}(5)-\mathrm{H}(5 \mathrm{~B})$ & 108.7 \\
\hline $\mathrm{C}(8)-\mathrm{H}(8 \mathrm{~B})$ & 0.9800 & $\mathrm{~N}(3)-\mathrm{C}(5)-\mathrm{H}(5 \mathrm{~B})$ & 108.7 \\
\hline \multirow[t]{2}{*}{$\mathrm{C}(8)-\mathrm{H}(8 \mathrm{C})$} & 0.9800 & $\mathrm{H}(5 \mathrm{~A})-\mathrm{C}(5)-\mathrm{H}(5 \mathrm{~B})$ & 107.6 \\
\hline & & $\mathrm{N}(3)-\mathrm{C}(6)-\mathrm{N}(1)$ & $114.4(3)$ \\
\hline $\mathrm{O}(3)-\mathrm{P}(1)-\mathrm{C}(2)$ & 117.36(19) & $\mathrm{N}(3)-\mathrm{C}(6)-\mathrm{H}(6 \mathrm{~A})$ & 108.7 \\
\hline $\mathrm{O}(3)-\mathrm{P}(1)-\mathrm{C}(3)$ & 117.92(18) & $\mathrm{N}(1)-\mathrm{C}(6)-\mathrm{H}(6 \mathrm{~A})$ & 108.7 \\
\hline $\mathrm{C}(2)-\mathrm{P}(1)-\mathrm{C}(3)$ & $100.85(18)$ & $\mathrm{N}(3)-\mathrm{C}(6)-\mathrm{H}(6 \mathrm{~B})$ & 108.7 \\
\hline $\mathrm{O}(3)-\mathrm{P}(1)-\mathrm{C}(1)$ & $117.55(18)$ & $\mathrm{N}(1)-\mathrm{C}(6)-\mathrm{H}(6 \mathrm{~B})$ & 108.7 \\
\hline $\mathrm{C}(2)-\mathrm{P}(1)-\mathrm{C}(1)$ & $97.85(18)$ & $\mathrm{H}(6 \mathrm{~A})-\mathrm{C}(6)-\mathrm{H}(6 \mathrm{~B})$ & 107.6 \\
\hline $\mathrm{C}(3)-\mathrm{P}(1)-\mathrm{C}(1)$ & $101.96(18)$ & $\mathrm{O}(1)-\mathrm{C}(7)-\mathrm{O}(2)$ & $124.1(4)$ \\
\hline $\mathrm{C}(7)-\mathrm{O}(2)-\mathrm{C}(8)$ & $116.9(4)$ & $\mathrm{O}(1)-\mathrm{C}(7)-\mathrm{C}(1)$ & $125.4(4)$ \\
\hline $\mathrm{C}(4)-\mathrm{N}(1)-\mathrm{C}(6)$ & $107.3(3)$ & $\mathrm{O}(2)-\mathrm{C}(7)-\mathrm{C}(1)$ & $110.4(4)$ \\
\hline $\mathrm{C}(4)-\mathrm{N}(1)-\mathrm{C}(1)$ & $109.0(3)$ & $\mathrm{O}(2)-\mathrm{C}(8)-\mathrm{H}(8 \mathrm{~A})$ & 109.5 \\
\hline $\mathrm{C}(6)-\mathrm{N}(1)-\mathrm{C}(1)$ & $114.1(3)$ & $\mathrm{O}(2)-\mathrm{C}(8)-\mathrm{H}(8 \mathrm{~B})$ & 109.5 \\
\hline $\mathrm{C}(5)-\mathrm{N}(2)-\mathrm{C}(4)$ & $108.0(3)$ & $\mathrm{H}(8 \mathrm{~A})-\mathrm{C}(8)-\mathrm{H}(8 \mathrm{~B})$ & 109.5 \\
\hline $\mathrm{C}(5)-\mathrm{N}(2)-\mathrm{C}(2)$ & $110.7(3)$ & $\mathrm{O}(2)-\mathrm{C}(8)-\mathrm{H}(8 \mathrm{C})$ & 109.5 \\
\hline $\mathrm{C}(4)-\mathrm{N}(2)-\mathrm{C}(2)$ & $112.0(3)$ & $\mathrm{H}(8 \mathrm{~A})-\mathrm{C}(8)-\mathrm{H}(8 \mathrm{C})$ & 109.5 \\
\hline $\mathrm{C}(6)-\mathrm{N}(3)-\mathrm{C}(5)$ & $109.1(3)$ & $\mathrm{H}(8 \mathrm{~B})-\mathrm{C}(8)-\mathrm{H}(8 \mathrm{C})$ & 109.5 \\
\hline $\mathrm{C}(6)-\mathrm{N}(3)-\mathrm{C}(3)$ & $110.9(3)$ & & \\
\hline
\end{tabular}

Symmetry transformations used to generate equivalent atoms: 
Table 2-S. Bond lengths $[\AA]$ and angles $\left[{ }^{\circ}\right]$ for PTA-C $\left(\mathrm{C}_{6} \mathrm{H}_{5}\right)_{2} \mathrm{OH}$.

\begin{tabular}{|c|c|c|c|}
\hline $\mathrm{P}(1)-\mathrm{C}(3)$ & $1.864(4)$ & $\mathrm{O}(2)-\mathrm{H}(2 \mathrm{C})$ & 0.8400 \\
\hline $\mathrm{P}(1)-\mathrm{C}(2)$ & $1.871(5)$ & $\mathrm{N}(4)-\mathrm{C}(25)$ & $1.327(6)$ \\
\hline $\mathrm{P}(1)-\mathrm{C}(1)$ & $1.880(4)$ & $\mathrm{N}(4)-\mathrm{C}(23)$ & $1.475(6)$ \\
\hline $\mathrm{O}(1)-\mathrm{C}(7)$ & $1.435(5)$ & $\mathrm{N}(4)-\mathrm{C}(20)$ & $1.479(6)$ \\
\hline $\mathrm{O}(1)-\mathrm{H}(1 \mathrm{~A})$ & 0.8400 & $\mathrm{~N}(5)-\mathrm{C}(21)$ & $1.453(6)$ \\
\hline $\mathrm{N}(1)-\mathrm{C}(4)$ & $1.470(6)$ & $\mathrm{N}(5)-\mathrm{C}(24)$ & $1.474(6)$ \\
\hline $\mathrm{N}(1)-\mathrm{C}(6)$ & $1.472(5)$ & $\mathrm{N}(5)-\mathrm{C}(23)$ & $1.488(6)$ \\
\hline $\mathrm{N}(1)-\mathrm{C}(1)$ & $1.494(5)$ & $\mathrm{N}(6)-\mathrm{C}(22)$ & $1.435(6)$ \\
\hline$N(2)-C(4)$ & $1.462(5)$ & $\mathrm{N}(6)-\mathrm{C}(24)$ & $1.495(6)$ \\
\hline $\mathrm{N}(2)-\mathrm{C}(2)$ & $1.471(6)$ & $\mathrm{N}(6)-\mathrm{C}(25)$ & $1.520(6)$ \\
\hline $\mathrm{N}(2)-\mathrm{C}(5)$ & $1.474(6)$ & $C(20)-C(26)$ & $1.540(6)$ \\
\hline $\mathrm{N}(3)-\mathrm{C}(6)$ & $1.467(5)$ & $\mathrm{C}(20)-\mathrm{H}(20 \mathrm{~A})$ & 1.0000 \\
\hline$N(3)-C(5)$ & $1.468(6)$ & $\mathrm{C}(21)-\mathrm{H}(21 \mathrm{~A})$ & 0.9900 \\
\hline $\mathrm{N}(3)-\mathrm{C}(3)$ & $1.474(5)$ & $\mathrm{C}(21)-\mathrm{H}(21 \mathrm{~B})$ & 0.9900 \\
\hline $\mathrm{C}(1)-\mathrm{C}(7)$ & $1.523(6)$ & $\mathrm{C}(22)-\mathrm{H}(22 \mathrm{~A})$ & 0.9900 \\
\hline $\mathrm{C}(1)-\mathrm{H}(1 \mathrm{~B})$ & 1.0000 & $\mathrm{C}(22)-\mathrm{H}(22 \mathrm{~B})$ & 0.9900 \\
\hline $\mathrm{C}(2)-\mathrm{H}(2 \mathrm{~A})$ & 0.9900 & $\mathrm{C}(23)-\mathrm{H}(23 \mathrm{~A})$ & 0.9900 \\
\hline $\mathrm{C}(2)-\mathrm{H}(2 \mathrm{~B})$ & 0.9900 & $\mathrm{C}(23)-\mathrm{H}(23 \mathrm{~B})$ & 0.9900 \\
\hline $\mathrm{C}(3)-\mathrm{H}(3 \mathrm{~A})$ & 0.9900 & $\mathrm{C}(24)-\mathrm{H}(24 \mathrm{~A})$ & 0.9900 \\
\hline C(3)-H(3B) & 0.9900 & $\mathrm{C}(24)-\mathrm{H}(24 \mathrm{~B})$ & 0.9900 \\
\hline $\mathrm{C}(4)-\mathrm{H}(4 \mathrm{~A})$ & 0.9900 & $\mathrm{C}(25)-\mathrm{H}(25 \mathrm{~A})$ & 0.9900 \\
\hline $\mathrm{C}(4)-\mathrm{H}(4 \mathrm{~B})$ & 0.9900 & $\mathrm{C}(25)-\mathrm{H}(25 \mathrm{~B})$ & 0.9900 \\
\hline $\mathrm{C}(5)-\mathrm{H}(5 \mathrm{~A})$ & 0.9900 & $\mathrm{C}(26)-\mathrm{C}(27)$ & $1.529(6)$ \\
\hline $\mathrm{C}(5)-\mathrm{H}(5 \mathrm{~B})$ & 0.9900 & $C(26)-C(33)$ & $1.540(6)$ \\
\hline $\mathrm{C}(6)-\mathrm{H}(6 \mathrm{~A})$ & 0.9900 & $C(27)-C(32)$ & $1.387(6)$ \\
\hline $\mathrm{C}(6)-\mathrm{H}(6 \mathrm{~B})$ & 0.9900 & $\mathrm{C}(27)-\mathrm{C}(28)$ & $1.389(6)$ \\
\hline$C(7)-C(14)$ & $1.541(6)$ & $\mathrm{C}(28)-\mathrm{C}(29)$ & $1.390(6)$ \\
\hline $\mathrm{C}(7)-\mathrm{C}(8)$ & $1.545(6)$ & $\mathrm{C}(28)-\mathrm{H}(28 \mathrm{~A})$ & 0.9500 \\
\hline $\mathrm{C}(8)-\mathrm{C}(9)$ & $1.389(6)$ & $\mathrm{C}(29)-\mathrm{C}(30)$ & $1.370(7)$ \\
\hline $\mathrm{C}(8)-\mathrm{C}(13)$ & $1.391(6)$ & $\mathrm{C}(29)-\mathrm{H}(29 \mathrm{~A})$ & 0.9500 \\
\hline $\mathrm{C}(9)-\mathrm{C}(10)$ & $1.391(7)$ & $\mathrm{C}(30)-\mathrm{C}(31)$ & $1.388(6)$ \\
\hline $\mathrm{C}(9)-\mathrm{H}(9 \mathrm{~A})$ & 0.9500 & $\mathrm{C}(30)-\mathrm{H}(30 \mathrm{~A})$ & 0.9500 \\
\hline C(10)-C(11) & $1.376(7)$ & $\mathrm{C}(31)-\mathrm{C}(32)$ & $1.374(6)$ \\
\hline $\mathrm{C}(10)-\mathrm{H}(10 \mathrm{~A})$ & 0.9500 & $\mathrm{C}(31)-\mathrm{H}(31 \mathrm{~A})$ & 0.9500 \\
\hline $\mathrm{C}(11)-\mathrm{C}(12)$ & $1.371(7)$ & $\mathrm{C}(32)-\mathrm{H}(32 \mathrm{~A})$ & 0.9500 \\
\hline $\mathrm{C}(11)-\mathrm{H}(11 \mathrm{~A})$ & 0.9500 & C(33)-C(38) & $1.390(6)$ \\
\hline C(12)-C(13) & $1.390(6)$ & $\mathrm{C}(33)-\mathrm{C}(34)$ & $1.389(6)$ \\
\hline $\mathrm{C}(12)-\mathrm{H}(12 \mathrm{~A})$ & 0.9500 & $\mathrm{C}(34)-\mathrm{C}(35)$ & $1.390(6)$ \\
\hline $\mathrm{C}(13)-\mathrm{H}(13 \mathrm{~A})$ & 0.9500 & $\mathrm{C}(34)-\mathrm{H}(34 \mathrm{~A})$ & 0.9500 \\
\hline$C(14)-C(15)$ & $1.381(6)$ & $C(35)-C(36)$ & $1.381(6)$ \\
\hline C(14)-C(19) & $1.391(6)$ & $\mathrm{C}(35)-\mathrm{H}(35 \mathrm{~A})$ & 0.9500 \\
\hline C(15)-C(16) & $1.388(6)$ & $\mathrm{C}(36)-\mathrm{C}(37)$ & $1.379(6)$ \\
\hline $\mathrm{C}(15)-\mathrm{H}(15 \mathrm{~A})$ & 0.9500 & $\mathrm{C}(36)-\mathrm{H}(36 \mathrm{~A})$ & 0.9500 \\
\hline$C(16)-C(17)$ & $1.383(7)$ & C(37)-C(38) & $1.391(6)$ \\
\hline $\mathrm{C}(16)-\mathrm{H}(16 \mathrm{~A})$ & 0.9500 & $\mathrm{C}(37)-\mathrm{H}(37 \mathrm{~A})$ & 0.9500 \\
\hline$C(17)-C(18)$ & $1.375(7)$ & $\mathrm{C}(38)-\mathrm{H}(38 \mathrm{~A})$ & 0.9500 \\
\hline $\mathrm{C}(17)-\mathrm{H}(17 \mathrm{~A})$ & 0.9500 & $\mathrm{C}(2 \mathrm{~S})-\mathrm{O}(1 \mathrm{SA}) \# 1$ & $1.06(5)$ \\
\hline $\mathrm{C}(18)-\mathrm{C}(19)$ & $1.385(6)$ & $\mathrm{C}(2 \mathrm{~S})-\mathrm{C}(3 \mathrm{~S})$ & $1.424(12)$ \\
\hline $\mathrm{C}(18)-\mathrm{H}(18 \mathrm{~A})$ & 0.9500 & $\mathrm{C}(2 \mathrm{~S})-\mathrm{O}(1 \mathrm{SB}) \# 1$ & $1.620(13)$ \\
\hline $\mathrm{C}(19)-\mathrm{H}(19 \mathrm{~A})$ & 0.9500 & $C(2 S)-C(3 S) \# 1$ & $1.969(13)$ \\
\hline $\mathrm{P}(2)-\mathrm{C}(22)$ & $1.855(5)$ & $\mathrm{C}(3 \mathrm{~S})-\mathrm{O}(1 \mathrm{SA})$ & $1.22(5)$ \\
\hline $\mathrm{P}(2)-\mathrm{C}(21)$ & $1.867(5)$ & $\mathrm{C}(3 \mathrm{~S})-\mathrm{O}(1 \mathrm{SB})$ & $1.369(13)$ \\
\hline$P(2)-C(20)$ & $1.917(4)$ & $\mathrm{C}(3 \mathrm{~S})-\mathrm{C}(2 \mathrm{~S}) \# 1$ & $1.969(13)$ \\
\hline $\mathrm{O}(2)-\mathrm{C}(26)$ & $1.435(5)$ & $\mathrm{O}(1 \mathrm{SB})-\mathrm{O}(1 \mathrm{SA})$ & $0.97(5)$ \\
\hline
\end{tabular}




\begin{tabular}{|c|c|c|c|}
\hline \multirow{3}{*}{$\begin{array}{l}\mathrm{O}(1 \mathrm{SB})-\mathrm{C}(2 \mathrm{~S}) \# 1 \\
\mathrm{O}(1 \mathrm{SA})-\mathrm{C}(2 \mathrm{~S}) \# 1\end{array}$} & $1.620(13)$ & $\mathrm{C}(1)-\mathrm{C}(7)-\mathrm{C}(8)$ & $112.1(4)$ \\
\hline & $1.06(5)$ & $C(14)-C(7)-C(8)$ & $109.5(3)$ \\
\hline & & $\mathrm{C}(9)-\mathrm{C}(8)-\mathrm{C}(13)$ & $117.3(4)$ \\
\hline $\mathrm{C}(3)-\mathrm{P}(1)-\mathrm{C}(2)$ & $94.7(2)$ & $\mathrm{C}(9)-\mathrm{C}(8)-\mathrm{C}(7)$ & $125.0(4)$ \\
\hline $\mathrm{C}(3)-\mathrm{P}(1)-\mathrm{C}(1)$ & $98.64(19)$ & $C(13)-C(8)-C(7)$ & $117.7(4)$ \\
\hline $\mathrm{C}(2)-\mathrm{P}(1)-\mathrm{C}(1)$ & $93.5(2)$ & $\mathrm{C}(8)-\mathrm{C}(9)-\mathrm{C}(10)$ & $121.0(5)$ \\
\hline $\mathrm{C}(7)-\mathrm{O}(1)-\mathrm{H}(1 \mathrm{~A})$ & 109.5 & $\mathrm{C}(8)-\mathrm{C}(9)-\mathrm{H}(9 \mathrm{~A})$ & 119.5 \\
\hline $\mathrm{C}(4)-\mathrm{N}(1)-\mathrm{C}(6)$ & 108.1(3) & $\mathrm{C}(10)-\mathrm{C}(9)-\mathrm{H}(9 \mathrm{~A})$ & 119.5 \\
\hline $\mathrm{C}(4)-\mathrm{N}(1)-\mathrm{C}(1)$ & 109.1(4) & $\mathrm{C}(11)-\mathrm{C}(10)-\mathrm{C}(9)$ & $120.3(5)$ \\
\hline $\mathrm{C}(6)-\mathrm{N}(1)-\mathrm{C}(1)$ & $113.8(3)$ & $\mathrm{C}(11)-\mathrm{C}(10)-\mathrm{H}(10 \mathrm{~A})$ & 119.9 \\
\hline $\mathrm{C}(4)-\mathrm{N}(2)-\mathrm{C}(2)$ & $110.5(3)$ & $\mathrm{C}(9)-\mathrm{C}(10)-\mathrm{H}(10 \mathrm{~A})$ & 119.9 \\
\hline $\mathrm{C}(4)-\mathrm{N}(2)-\mathrm{C}(5)$ & $107.2(4)$ & $\mathrm{C}(12)-\mathrm{C}(11)-\mathrm{C}(10)$ & $119.9(5)$ \\
\hline $\mathrm{C}(2)-\mathrm{N}(2)-\mathrm{C}(5)$ & $110.6(3)$ & $\mathrm{C}(12)-\mathrm{C}(11)-\mathrm{H}(11 \mathrm{~A})$ & 120.0 \\
\hline$C(6)-N(3)-C(5)$ & $108.9(3)$ & $\mathrm{C}(10)-\mathrm{C}(11)-\mathrm{H}(11 \mathrm{~A})$ & 120.0 \\
\hline$C(6)-N(3)-C(3)$ & 109.9(3) & $\mathrm{C}(11)-\mathrm{C}(12)-\mathrm{C}(13)$ & $119.6(5)$ \\
\hline $\mathrm{C}(5)-\mathrm{N}(3)-\mathrm{C}(3)$ & $110.4(4)$ & $\mathrm{C}(11)-\mathrm{C}(12)-\mathrm{H}(12 \mathrm{~A})$ & 120.2 \\
\hline $\mathrm{N}(1)-\mathrm{C}(1)-\mathrm{C}(7)$ & $114.9(3)$ & $\mathrm{C}(13)-\mathrm{C}(12)-\mathrm{H}(12 \mathrm{~A})$ & 120.2 \\
\hline $\mathrm{N}(1)-\mathrm{C}(1)-\mathrm{P}(1)$ & $112.4(3)$ & $\mathrm{C}(12)-\mathrm{C}(13)-\mathrm{C}(8)$ & $121.8(5)$ \\
\hline $\mathrm{C}(7)-\mathrm{C}(1)-\mathrm{P}(1)$ & $112.8(3)$ & $\mathrm{C}(12)-\mathrm{C}(13)-\mathrm{H}(13 \mathrm{~A})$ & 119.1 \\
\hline $\mathrm{N}(1)-\mathrm{C}(1)-\mathrm{H}(1 \mathrm{~B})$ & 105.2 & $\mathrm{C}(8)-\mathrm{C}(13)-\mathrm{H}(13 \mathrm{~A})$ & 119.1 \\
\hline $\mathrm{C}(7)-\mathrm{C}(1)-\mathrm{H}(1 \mathrm{~B})$ & 105.2 & $\mathrm{C}(15)-\mathrm{C}(14)-\mathrm{C}(19)$ & $118.7(4)$ \\
\hline $\mathrm{P}(1)-\mathrm{C}(1)-\mathrm{H}(1 \mathrm{~B})$ & 105.2 & $C(15)-C(14)-C(7)$ & $121.3(4)$ \\
\hline $\mathrm{N}(2)-\mathrm{C}(2)-\mathrm{P}(1)$ & $115.8(3)$ & $C(19)-C(14)-C(7)$ & $120.0(4)$ \\
\hline $\mathrm{N}(2)-\mathrm{C}(2)-\mathrm{H}(2 \mathrm{~A})$ & 108.3 & $C(14)-C(15)-C(16)$ & $121.0(4)$ \\
\hline $\mathrm{P}(1)-\mathrm{C}(2)-\mathrm{H}(2 \mathrm{~A})$ & 108.3 & $\mathrm{C}(14)-\mathrm{C}(15)-\mathrm{H}(15 \mathrm{~A})$ & 119.5 \\
\hline $\mathrm{N}(2)-\mathrm{C}(2)-\mathrm{H}(2 \mathrm{~B})$ & 108.3 & $\mathrm{C}(16)-\mathrm{C}(15)-\mathrm{H}(15 \mathrm{~A})$ & 119.5 \\
\hline $\mathrm{P}(1)-\mathrm{C}(2)-\mathrm{H}(2 \mathrm{~B})$ & 108.3 & $\mathrm{C}(17)-\mathrm{C}(16)-\mathrm{C}(15)$ & $119.4(5)$ \\
\hline $\mathrm{H}(2 \mathrm{~A})-\mathrm{C}(2)-\mathrm{H}(2 \mathrm{~B})$ & 107.4 & $\mathrm{C}(17)-\mathrm{C}(16)-\mathrm{H}(16 \mathrm{~A})$ & 120.3 \\
\hline $\mathrm{N}(3)-\mathrm{C}(3)-\mathrm{P}(1)$ & $115.3(3)$ & $\mathrm{C}(15)-\mathrm{C}(16)-\mathrm{H}(16 \mathrm{~A})$ & 120.3 \\
\hline $\mathrm{N}(3)-\mathrm{C}(3)-\mathrm{H}(3 \mathrm{~A})$ & 108.5 & $\mathrm{C}(18)-\mathrm{C}(17)-\mathrm{C}(16)$ & $120.3(4)$ \\
\hline $\mathrm{P}(1)-\mathrm{C}(3)-\mathrm{H}(3 \mathrm{~A})$ & 108.5 & $\mathrm{C}(18)-\mathrm{C}(17)-\mathrm{H}(17 \mathrm{~A})$ & 119.8 \\
\hline N(3)-C(3)-H(3B) & 108.5 & $\mathrm{C}(16)-\mathrm{C}(17)-\mathrm{H}(17 \mathrm{~A})$ & 119.8 \\
\hline $\mathrm{P}(1)-\mathrm{C}(3)-\mathrm{H}(3 \mathrm{~B})$ & 108.5 & $\mathrm{C}(17)-\mathrm{C}(18)-\mathrm{C}(19)$ & $119.9(4)$ \\
\hline $\mathrm{H}(3 \mathrm{~A})-\mathrm{C}(3)-\mathrm{H}(3 \mathrm{~B})$ & 107.5 & $\mathrm{C}(17)-\mathrm{C}(18)-\mathrm{H}(18 \mathrm{~A})$ & 120.1 \\
\hline $\mathrm{N}(2)-\mathrm{C}(4)-\mathrm{N}(1)$ & $115.3(3)$ & $\mathrm{C}(19)-\mathrm{C}(18)-\mathrm{H}(18 \mathrm{~A})$ & 120.1 \\
\hline $\mathrm{N}(2)-\mathrm{C}(4)-\mathrm{H}(4 \mathrm{~A})$ & 108.5 & $\mathrm{C}(18)-\mathrm{C}(19)-\mathrm{C}(14)$ & $120.7(4)$ \\
\hline $\mathrm{N}(1)-\mathrm{C}(4)-\mathrm{H}(4 \mathrm{~A})$ & 108.5 & $\mathrm{C}(18)-\mathrm{C}(19)-\mathrm{H}(19 \mathrm{~A})$ & 119.7 \\
\hline $\mathrm{N}(2)-\mathrm{C}(4)-\mathrm{H}(4 \mathrm{~B})$ & 108.5 & $\mathrm{C}(14)-\mathrm{C}(19)-\mathrm{H}(19 \mathrm{~A})$ & 119.7 \\
\hline $\mathrm{N}(1)-\mathrm{C}(4)-\mathrm{H}(4 \mathrm{~B})$ & 108.5 & $\mathrm{C}(22)-\mathrm{P}(2)-\mathrm{C}(21)$ & $94.2(2)$ \\
\hline $\mathrm{H}(4 \mathrm{~A})-\mathrm{C}(4)-\mathrm{H}(4 \mathrm{~B})$ & 107.5 & $\mathrm{C}(22)-\mathrm{P}(2)-\mathrm{C}(20)$ & $99.2(2)$ \\
\hline $\mathrm{N}(3)-\mathrm{C}(5)-\mathrm{N}(2)$ & $115.0(3)$ & $\mathrm{C}(21)-\mathrm{P}(2)-\mathrm{C}(20)$ & $92.0(2)$ \\
\hline $\mathrm{N}(3)-\mathrm{C}(5)-\mathrm{H}(5 \mathrm{~A})$ & 108.5 & $\mathrm{C}(26)-\mathrm{O}(2)-\mathrm{H}(2 \mathrm{C})$ & 109.5 \\
\hline $\mathrm{N}(2)-\mathrm{C}(5)-\mathrm{H}(5 \mathrm{~A})$ & 108.5 & $\mathrm{C}(25)-\mathrm{N}(4)-\mathrm{C}(23)$ & $108.2(4)$ \\
\hline $\mathrm{N}(3)-\mathrm{C}(5)-\mathrm{H}(5 \mathrm{~B})$ & 108.5 & $\mathrm{C}(25)-\mathrm{N}(4)-\mathrm{C}(20)$ & $118.0(4)$ \\
\hline $\mathrm{N}(2)-\mathrm{C}(5)-\mathrm{H}(5 \mathrm{~B})$ & 108.5 & $\mathrm{C}(23)-\mathrm{N}(4)-\mathrm{C}(20)$ & $108.6(4)$ \\
\hline $\mathrm{H}(5 \mathrm{~A})-\mathrm{C}(5)-\mathrm{H}(5 \mathrm{~B})$ & 107.5 & $\mathrm{C}(21)-\mathrm{N}(5)-\mathrm{C}(24)$ & $111.8(4)$ \\
\hline $\mathrm{N}(3)-\mathrm{C}(6)-\mathrm{N}(1)$ & $114.6(4)$ & $\mathrm{C}(21)-\mathrm{N}(5)-\mathrm{C}(23)$ & $111.0(4)$ \\
\hline $\mathrm{N}(3)-\mathrm{C}(6)-\mathrm{H}(6 \mathrm{~A})$ & 108.6 & $\mathrm{C}(24)-\mathrm{N}(5)-\mathrm{C}(23)$ & $104.7(4)$ \\
\hline $\mathrm{N}(1)-\mathrm{C}(6)-\mathrm{H}(6 \mathrm{~A})$ & 108.6 & $\mathrm{C}(22)-\mathrm{N}(6)-\mathrm{C}(24)$ & $108.0(4)$ \\
\hline $\mathrm{N}(3)-\mathrm{C}(6)-\mathrm{H}(6 \mathrm{~B})$ & 108.6 & $\mathrm{C}(22)-\mathrm{N}(6)-\mathrm{C}(25)$ & 111.4(4) \\
\hline $\mathrm{N}(1)-\mathrm{C}(6)-\mathrm{H}(6 \mathrm{~B})$ & 108.6 & $\mathrm{C}(24)-\mathrm{N}(6)-\mathrm{C}(25)$ & $106.4(4)$ \\
\hline $\mathrm{H}(6 \mathrm{~A})-\mathrm{C}(6)-\mathrm{H}(6 \mathrm{~B})$ & 107.6 & $\mathrm{~N}(4)-\mathrm{C}(20)-\mathrm{C}(26)$ & $114.5(4)$ \\
\hline $\mathrm{O}(1)-\mathrm{C}(7)-\mathrm{C}(1)$ & $106.9(3)$ & $\mathrm{N}(4)-\mathrm{C}(20)-\mathrm{P}(2)$ & $110.6(3)$ \\
\hline $\mathrm{O}(1)-\mathrm{C}(7)-\mathrm{C}(14)$ & $109.9(3)$ & $\mathrm{C}(26)-\mathrm{C}(20)-\mathrm{P}(2)$ & 113.1(3) \\
\hline $\mathrm{C}(1)-\mathrm{C}(7)-\mathrm{C}(14)$ & $109.5(3)$ & $\mathrm{N}(4)-\mathrm{C}(20)-\mathrm{H}(20 \mathrm{~A})$ & 105.9 \\
\hline $\mathrm{O}(1)-\mathrm{C}(7)-\mathrm{C}(8)$ & $108.8(3)$ & $\mathrm{C}(26)-\mathrm{C}(20)-\mathrm{H}(20 \mathrm{~A})$ & 105.9 \\
\hline
\end{tabular}




\begin{tabular}{|c|c|}
\hline$P(2)-C(20)-H(20 A)$ & 105.9 \\
\hline $\mathrm{N}(5)-\mathrm{C}(21)-\mathrm{P}(2)$ & $116.1(3)$ \\
\hline $\mathrm{N}(5)-\mathrm{C}(21)-\mathrm{H}(21 \mathrm{~A})$ & 108.3 \\
\hline $\mathrm{P}(2)-\mathrm{C}(21)-\mathrm{H}(21 \mathrm{~A})$ & 108.3 \\
\hline $\mathrm{N}(5)-\mathrm{C}(21)-\mathrm{H}(21 \mathrm{~B})$ & 108.3 \\
\hline $\mathrm{P}(2)-\mathrm{C}(21)-\mathrm{H}(21 \mathrm{~B})$ & 108.3 \\
\hline $\mathrm{H}(21 \mathrm{~A})-\mathrm{C}(21)-\mathrm{H}(21 \mathrm{~B})$ & 107.4 \\
\hline $\mathrm{N}(6)-\mathrm{C}(22)-\mathrm{P}(2)$ & $116.5(3)$ \\
\hline $\mathrm{N}(6)-\mathrm{C}(22)-\mathrm{H}(22 \mathrm{~A})$ & 108.2 \\
\hline $\mathrm{P}(2)-\mathrm{C}(22)-\mathrm{H}(22 \mathrm{~A})$ & 108.2 \\
\hline $\mathrm{N}(6)-\mathrm{C}(22)-\mathrm{H}(22 \mathrm{~B})$ & 108.2 \\
\hline $\mathrm{P}(2)-\mathrm{C}(22)-\mathrm{H}(22 \mathrm{~B})$ & 108.2 \\
\hline $\mathrm{H}(22 \mathrm{~A})-\mathrm{C}(22)-\mathrm{H}(22 \mathrm{~B})$ & 107.3 \\
\hline $\mathrm{N}(4)-\mathrm{C}(23)-\mathrm{N}(5)$ & $114.5(4)$ \\
\hline $\mathrm{N}(4)-\mathrm{C}(23)-\mathrm{H}(23 \mathrm{~A})$ & 108.6 \\
\hline $\mathrm{N}(5)-\mathrm{C}(23)-\mathrm{H}(23 \mathrm{~A})$ & 108.6 \\
\hline $\mathrm{N}(4)-\mathrm{C}(23)-\mathrm{H}(23 \mathrm{~B})$ & 108.6 \\
\hline $\mathrm{N}(5)-\mathrm{C}(23)-\mathrm{H}(23 \mathrm{~B})$ & 108.6 \\
\hline $\mathrm{H}(23 \mathrm{~A})-\mathrm{C}(23)-\mathrm{H}(23 \mathrm{~B})$ & 107.6 \\
\hline $\mathrm{N}(5)-\mathrm{C}(24)-\mathrm{N}(6)$ & $114.7(4)$ \\
\hline $\mathrm{N}(5)-\mathrm{C}(24)-\mathrm{H}(24 \mathrm{~A})$ & 108.6 \\
\hline $\mathrm{N}(6)-\mathrm{C}(24)-\mathrm{H}(24 \mathrm{~A})$ & 108.6 \\
\hline $\mathrm{N}(5)-\mathrm{C}(24)-\mathrm{H}(24 \mathrm{~B})$ & 108.6 \\
\hline $\mathrm{N}(6)-\mathrm{C}(24)-\mathrm{H}(24 \mathrm{~B})$ & 108.6 \\
\hline $\mathrm{H}(24 \mathrm{~A})-\mathrm{C}(24)-\mathrm{H}(24 \mathrm{~B})$ & 107.6 \\
\hline $\mathrm{N}(4)-\mathrm{C}(25)-\mathrm{N}(6)$ & $116.8(4)$ \\
\hline $\mathrm{N}(4)-\mathrm{C}(25)-\mathrm{H}(25 \mathrm{~A})$ & 108.1 \\
\hline $\mathrm{N}(6)-\mathrm{C}(25)-\mathrm{H}(25 \mathrm{~A})$ & 108.1 \\
\hline $\mathrm{N}(4)-\mathrm{C}(25)-\mathrm{H}(25 \mathrm{~B})$ & 108.1 \\
\hline $\mathrm{N}(6)-\mathrm{C}(25)-\mathrm{H}(25 \mathrm{~B})$ & 108.1 \\
\hline $\mathrm{H}(25 \mathrm{~A})-\mathrm{C}(25)-\mathrm{H}(25 \mathrm{~B})$ & 107.3 \\
\hline $\mathrm{O}(2)-\mathrm{C}(26)-\mathrm{C}(27)$ & $108.7(3)$ \\
\hline $\mathrm{O}(2)-\mathrm{C}(26)-\mathrm{C}(20)$ & $110.8(3)$ \\
\hline$C(27)-C(26)-C(20)$ & $113.1(3)$ \\
\hline $\mathrm{O}(2)-\mathrm{C}(26)-\mathrm{C}(33)$ & $108.4(3)$ \\
\hline $\mathrm{C}(27)-\mathrm{C}(26)-\mathrm{C}(33)$ & $107.4(3)$ \\
\hline$C(20)-C(26)-C(33)$ & $108.2(3)$ \\
\hline $\mathrm{C}(32)-\mathrm{C}(27)-\mathrm{C}(28)$ & $117.6(4)$ \\
\hline$C(32)-C(27)-C(26)$ & $116.3(4)$ \\
\hline $\mathrm{C}(28)-\mathrm{C}(27)-\mathrm{C}(26)$ & $126.0(4)$ \\
\hline $\mathrm{C}(27)-\mathrm{C}(28)-\mathrm{C}(29)$ & $121.5(4)$ \\
\hline $\mathrm{C}(27)-\mathrm{C}(28)-\mathrm{H}(28 \mathrm{~A})$ & 119.3 \\
\hline $\mathrm{C}(29)-\mathrm{C}(28)-\mathrm{H}(28 \mathrm{~A})$ & 119.3 \\
\hline $\mathrm{C}(30)-\mathrm{C}(29)-\mathrm{C}(28)$ & $119.5(4)$ \\
\hline $\mathrm{C}(30)-\mathrm{C}(29)-\mathrm{H}(29 \mathrm{~A})$ & 120.3 \\
\hline $\mathrm{C}(28)-\mathrm{C}(29)-\mathrm{H}(29 \mathrm{~A})$ & 120.3 \\
\hline
\end{tabular}

\begin{tabular}{|c|c|}
\hline$C(29)-C(30)-C(31)$ & $120.1(4)$ \\
\hline $\mathrm{C}(29)-\mathrm{C}(30)-\mathrm{H}(30 \mathrm{~A})$ & 119.9 \\
\hline $\mathrm{C}(31)-\mathrm{C}(30)-\mathrm{H}(30 \mathrm{~A})$ & 119.9 \\
\hline $\mathrm{C}(32)-\mathrm{C}(31)-\mathrm{C}(30)$ & $119.8(4)$ \\
\hline $\mathrm{C}(32)-\mathrm{C}(31)-\mathrm{H}(31 \mathrm{~A})$ & 120.1 \\
\hline $\mathrm{C}(30)-\mathrm{C}(31)-\mathrm{H}(31 \mathrm{~A})$ & 120.1 \\
\hline $\mathrm{C}(31)-\mathrm{C}(32)-\mathrm{C}(27)$ & $121.5(4)$ \\
\hline $\mathrm{C}(31)-\mathrm{C}(32)-\mathrm{H}(32 \mathrm{~A})$ & 119.3 \\
\hline $\mathrm{C}(27)-\mathrm{C}(32)-\mathrm{H}(32 \mathrm{~A})$ & 119.3 \\
\hline $\mathrm{C}(38)-\mathrm{C}(33)-\mathrm{C}(34)$ & $118.9(4)$ \\
\hline $\mathrm{C}(38)-\mathrm{C}(33)-\mathrm{C}(26)$ & $119.6(4)$ \\
\hline $\mathrm{C}(34)-\mathrm{C}(33)-\mathrm{C}(26)$ & $121.6(4)$ \\
\hline $\mathrm{C}(33)-\mathrm{C}(34)-\mathrm{C}(35)$ & $119.8(4)$ \\
\hline $\mathrm{C}(33)-\mathrm{C}(34)-\mathrm{H}(34 \mathrm{~A})$ & 120.1 \\
\hline $\mathrm{C}(35)-\mathrm{C}(34)-\mathrm{H}(34 \mathrm{~A})$ & 120.1 \\
\hline $\mathrm{C}(36)-\mathrm{C}(35)-\mathrm{C}(34)$ & $121.0(4)$ \\
\hline $\mathrm{C}(36)-\mathrm{C}(35)-\mathrm{H}(35 \mathrm{~A})$ & 119.5 \\
\hline $\mathrm{C}(34)-\mathrm{C}(35)-\mathrm{H}(35 \mathrm{~A})$ & 119.5 \\
\hline $\mathrm{C}(37)-\mathrm{C}(36)-\mathrm{C}(35)$ & $119.6(4)$ \\
\hline $\mathrm{C}(37)-\mathrm{C}(36)-\mathrm{H}(36 \mathrm{~A})$ & 120.2 \\
\hline $\mathrm{C}(35)-\mathrm{C}(36)-\mathrm{H}(36 \mathrm{~A})$ & 120.2 \\
\hline $\mathrm{C}(36)-\mathrm{C}(37)-\mathrm{C}(38)$ & $119.8(4)$ \\
\hline $\mathrm{C}(36)-\mathrm{C}(37)-\mathrm{H}(37 \mathrm{~A})$ & 120.1 \\
\hline $\mathrm{C}(38)-\mathrm{C}(37)-\mathrm{H}(37 \mathrm{~A})$ & 120.1 \\
\hline $\mathrm{C}(33)-\mathrm{C}(38)-\mathrm{C}(37)$ & $120.9(4)$ \\
\hline $\mathrm{C}(33)-\mathrm{C}(38)-\mathrm{H}(38 \mathrm{~A})$ & 119.5 \\
\hline $\mathrm{C}(37)-\mathrm{C}(38)-\mathrm{H}(38 \mathrm{~A})$ & 119.5 \\
\hline $\mathrm{O}(1 \mathrm{SA}) \# 1-\mathrm{C}(2 \mathrm{~S})-\mathrm{C}(3 \mathrm{~S})$ & $121(2)$ \\
\hline $\mathrm{O}(1 \mathrm{SA}) \# 1-\mathrm{C}(2 \mathrm{~S})-\mathrm{O}(1 \mathrm{SB}) \# 1$ & $35(3)$ \\
\hline $\mathrm{C}(3 \mathrm{~S})-\mathrm{C}(2 \mathrm{~S})-\mathrm{O}(1 \mathrm{SB}) \# 1$ & $124.0(7)$ \\
\hline $\mathrm{O}(1 \mathrm{SA}) \# 1-\mathrm{C}(2 \mathrm{~S})-\mathrm{C}(3 \mathrm{~S}) \# 1$ & $33(2)$ \\
\hline$C(3 S)-C(2 S)-C(3 S) \# 1$ & $90.1(7)$ \\
\hline $\mathrm{O}(1 \mathrm{SB}) \# 1-\mathrm{C}(2 \mathrm{~S})-\mathrm{C}(3 \mathrm{~S}) \# 1$ & $43.5(5)$ \\
\hline $\mathrm{O}(1 \mathrm{SA})-\mathrm{C}(3 \mathrm{~S})-\mathrm{O}(1 \mathrm{SB})$ & $43(2)$ \\
\hline $\mathrm{O}(1 \mathrm{SA})-\mathrm{C}(3 \mathrm{~S})-\mathrm{C}(2 \mathrm{~S})$ & $116(2)$ \\
\hline $\mathrm{O}(1 \mathrm{SB})-\mathrm{C}(3 \mathrm{~S})-\mathrm{C}(2 \mathrm{~S})$ & $131.2(10)$ \\
\hline $\mathrm{O}(1 \mathrm{SA})-\mathrm{C}(3 \mathrm{~S})-\mathrm{C}(2 \mathrm{~S}) \# 1$ & $28.2(19)$ \\
\hline $\mathrm{O}(1 \mathrm{SB})-\mathrm{C}(3 \mathrm{~S})-\mathrm{C}(2 \mathrm{~S}) \# 1$ & $54.6(6)$ \\
\hline$C(2 S)-C(3 S)-C(2 S) \# 1$ & $89.9(7)$ \\
\hline $\mathrm{O}(1 \mathrm{SA})-\mathrm{O}(1 \mathrm{SB})-\mathrm{C}(3 \mathrm{~S})$ & $60(3)$ \\
\hline $\mathrm{O}(1 \mathrm{SA})-\mathrm{O}(1 \mathrm{SB})-\mathrm{C}(2 \mathrm{~S}) \# 1$ & $39(3)$ \\
\hline $\mathrm{C}(3 \mathrm{~S})-\mathrm{O}(1 \mathrm{SB})-\mathrm{C}(2 \mathrm{~S}) \# 1$ & $81.9(9)$ \\
\hline $\mathrm{O}(1 \mathrm{SB})-\mathrm{O}(1 \mathrm{SA})-\mathrm{C}(2 \mathrm{~S}) \# 1$ & $106(5)$ \\
\hline $\mathrm{O}(1 \mathrm{SB})-\mathrm{O}(1 \mathrm{SA})-\mathrm{C}(3 \mathrm{~S})$ & $77(4)$ \\
\hline $\mathrm{C}(2 \mathrm{~S}) \# 1-\mathrm{O}(1 \mathrm{SA})-\mathrm{C}(3 \mathrm{~S})$ & $119(2)$ \\
\hline
\end{tabular}

Symmetry transformations used to generate equivalent atoms:

\#1 - x+2,-y+1,-z 
Table 3-S. Bond lengths $[\AA ̊]$ and angles $\left[{ }^{\circ}\right]$ for $\mathrm{O}=\mathrm{PTA}-\mathrm{C}\left(\mathrm{C}_{6} \mathrm{H}_{4} \mathrm{OCH}_{3}\right)_{2} \mathrm{OH}$.

\begin{tabular}{|c|c|c|c|}
\hline $\mathrm{P}(1)-\mathrm{O}(1)$ & $1.4926(14)$ & $C(7 S)-C(8 S)$ & $1.491(4)$ \\
\hline $\mathrm{P}(1)-\mathrm{C}(3)$ & $1.817(2)$ & $\mathrm{C}(7 \mathrm{~S})-\mathrm{H}(13)$ & 0.9900 \\
\hline$P(1)-C(2)$ & $1.822(2)$ & $\mathrm{C}(7 \mathrm{~S})-\mathrm{H}(14)$ & 0.9900 \\
\hline $\mathrm{P}(1)-\mathrm{C}(1)$ & $1.838(2)$ & $\mathrm{C}(8)-\mathrm{C}(13)$ & $1.383(3)$ \\
\hline $\mathrm{O}(1 \mathrm{~S})-\mathrm{C}(4 \mathrm{~S})$ & $1.432(3)$ & $C(8)-C(9)$ & $1.402(3)$ \\
\hline $\mathrm{O}(1 \mathrm{~S})-\mathrm{C}(1 \mathrm{~S})$ & $1.439(3)$ & $\mathrm{C}(8 \mathrm{~S})-\mathrm{H}(15)$ & 0.9900 \\
\hline $\mathrm{O}(2)-\mathrm{C}(7)$ & $1.425(2)$ & $\mathrm{C}(8 \mathrm{~S})-\mathrm{H}(16)$ & 0.9900 \\
\hline $\mathrm{O}(2)-\mathrm{H}(2 \mathrm{~A})$ & 0.8400 & $C(9)-C(10)$ & $1.377(3)$ \\
\hline $\mathrm{O}(2 \mathrm{~S})-\mathrm{C}(8 \mathrm{~S})$ & $1.415(3)$ & $\mathrm{C}(9)-\mathrm{H}(9 \mathrm{~A})$ & 0.9500 \\
\hline $\mathrm{O}(2 \mathrm{~S})-\mathrm{C}(5 \mathrm{~S})$ & $1.424(3)$ & $C(10)-C(11)$ & $1.391(3)$ \\
\hline $\mathrm{O}(3)-\mathrm{C}(11)$ & $1.369(2)$ & $\mathrm{C}(10)-\mathrm{H}(10 \mathrm{~A})$ & 0.9500 \\
\hline $\mathrm{O}(3)-\mathrm{C}(14)$ & $1.427(2)$ & $C(11)-C(12)$ & $1.384(3)$ \\
\hline $\mathrm{O}(4)-\mathrm{C}(18)$ & $1.371(2)$ & $\mathrm{C}(12)-\mathrm{C}(13)$ & $1.391(3)$ \\
\hline $\mathrm{O}(4)-\mathrm{C}(21)$ & $1.426(3)$ & $\mathrm{C}(12)-\mathrm{H}(12 \mathrm{~A})$ & 0.9500 \\
\hline $\mathrm{N}(1)-\mathrm{C}(6)$ & $1.469(2)$ & $\mathrm{C}(13)-\mathrm{H}(13 \mathrm{~A})$ & 0.9500 \\
\hline $\mathrm{N}(1)-\mathrm{C}(4)$ & $1.470(2)$ & $\mathrm{C}(14)-\mathrm{H}(14 \mathrm{~A})$ & 0.9800 \\
\hline $\mathrm{N}(1)-\mathrm{C}(1)$ & $1.490(2)$ & $\mathrm{C}(14)-\mathrm{H}(14 \mathrm{~B})$ & 0.9800 \\
\hline $\mathrm{N}(2)-\mathrm{C}(4)$ & $1.469(3)$ & $\mathrm{C}(14)-\mathrm{H}(14 \mathrm{C})$ & 0.9800 \\
\hline $\mathrm{N}(2)-\mathrm{C}(5)$ & $1.469(3)$ & $C(15)-C(16)$ & $1.386(3)$ \\
\hline $\mathrm{N}(2)-\mathrm{C}(2)$ & $1.483(3)$ & $C(15)-C(20)$ & $1.401(3)$ \\
\hline $\mathrm{N}(3)-\mathrm{C}(6)$ & $1.476(3)$ & $C(16)-C(17)$ & $1.393(3)$ \\
\hline $\mathrm{N}(3)-\mathrm{C}(3)$ & $1.476(3)$ & $\mathrm{C}(16)-\mathrm{H}(16 \mathrm{~A})$ & 0.9500 \\
\hline $\mathrm{N}(3)-\mathrm{C}(5)$ & $1.476(3)$ & $\mathrm{C}(17)-\mathrm{C}(18)$ & $1.384(3)$ \\
\hline $\mathrm{C}(1)-\mathrm{C}(7)$ & $1.562(3)$ & $\mathrm{C}(17)-\mathrm{H}(17 \mathrm{~A})$ & 0.9500 \\
\hline $\mathrm{C}(1)-\mathrm{H}(1 \mathrm{~B})$ & 1.0000 & $\mathrm{C}(18)-\mathrm{C}(19)$ & $1.393(3)$ \\
\hline$C(1 S)-C(2 S)$ & $1.507(4)$ & $C(19)-C(20)$ & $1.379(3)$ \\
\hline $\mathrm{C}(1 \mathrm{~S})-\mathrm{H}(1)$ & 0.9900 & $\mathrm{C}(19)-\mathrm{H}(19 \mathrm{~A})$ & 0.9500 \\
\hline $\mathrm{C}(1 \mathrm{~S})-\mathrm{H}(2)$ & 0.9900 & $\mathrm{C}(20)-\mathrm{H}(20 \mathrm{~A})$ & 0.9500 \\
\hline $\mathrm{C}(2)-\mathrm{H}(2 \mathrm{~B})$ & 0.9900 & $\mathrm{C}(21)-\mathrm{H}(21 \mathrm{~A})$ & 0.9800 \\
\hline $\mathrm{C}(2)-\mathrm{H}(2 \mathrm{C})$ & 0.9900 & $\mathrm{C}(21)-\mathrm{H}(21 \mathrm{~B})$ & 0.9800 \\
\hline$C(2 S)-C(3 S)$ & $1.509(4)$ & $\mathrm{C}(21)-\mathrm{H}(21 \mathrm{C})$ & 0.9800 \\
\hline $\mathrm{C}(2 \mathrm{~S})-\mathrm{H}(3)$ & 0.9900 & & \\
\hline $\mathrm{C}(2 \mathrm{~S})-\mathrm{H}(4)$ & 0.9900 & $\mathrm{O}(1)-\mathrm{P}(1)-\mathrm{C}(3)$ & 112.81(9) \\
\hline $\mathrm{C}(3)-\mathrm{H}(3 \mathrm{~A})$ & 0.9900 & $\mathrm{O}(1)-\mathrm{P}(1)-\mathrm{C}(2)$ & 119.14(9) \\
\hline $\mathrm{C}(3)-\mathrm{H}(3 \mathrm{~B})$ & 0.9900 & $\mathrm{C}(3)-\mathrm{P}(1)-\mathrm{C}(2)$ & $99.25(9)$ \\
\hline$C(3 S)-C(4 S)$ & $1.528(4)$ & $\mathrm{O}(1)-\mathrm{P}(1)-\mathrm{C}(1)$ & $119.09(8)$ \\
\hline $\mathrm{C}(3 \mathrm{~S})-\mathrm{H}(5)$ & 0.9900 & $\mathrm{C}(3)-\mathrm{P}(1)-\mathrm{C}(1)$ & $100.39(9)$ \\
\hline $\mathrm{C}(3 \mathrm{~S})-\mathrm{H}(6)$ & 0.9900 & $\mathrm{C}(2)-\mathrm{P}(1)-\mathrm{C}(1)$ & $102.87(9)$ \\
\hline $\mathrm{C}(4)-\mathrm{H}(4 \mathrm{~A})$ & 0.9900 & $\mathrm{C}(4 \mathrm{~S})-\mathrm{O}(1 \mathrm{~S})-\mathrm{C}(1 \mathrm{~S})$ & $108.84(18)$ \\
\hline $\mathrm{C}(4)-\mathrm{H}(4 \mathrm{~B})$ & 0.9900 & $\mathrm{C}(7)-\mathrm{O}(2)-\mathrm{H}(2 \mathrm{~A})$ & 109.5 \\
\hline $\mathrm{C}(4 \mathrm{~S})-\mathrm{H}(7)$ & 0.9900 & $\mathrm{C}(8 \mathrm{~S})-\mathrm{O}(2 \mathrm{~S})-\mathrm{C}(5 \mathrm{~S})$ & $108.90(19)$ \\
\hline $\mathrm{C}(4 \mathrm{~S})-\mathrm{H}(8)$ & 0.9900 & $\mathrm{C}(11)-\mathrm{O}(3)-\mathrm{C}(14)$ & $117.03(16)$ \\
\hline $\mathrm{C}(5)-\mathrm{H}(5 \mathrm{~B})$ & 0.9900 & $\mathrm{C}(18)-\mathrm{O}(4)-\mathrm{C}(21)$ & $116.48(16)$ \\
\hline $\mathrm{C}(5)-\mathrm{H}(5 \mathrm{C})$ & 0.9900 & $C(6)-N(1)-C(4)$ & $107.71(15)$ \\
\hline$C(5 S)-C(6 S)$ & $1.508(4)$ & $\mathrm{C}(6)-\mathrm{N}(1)-\mathrm{C}(1)$ & $112.37(15)$ \\
\hline $\mathrm{C}(5 \mathrm{~S})-\mathrm{H}(9)$ & 0.9900 & $\mathrm{C}(4)-\mathrm{N}(1)-\mathrm{C}(1)$ & $114.45(15)$ \\
\hline $\mathrm{C}(5 \mathrm{~S})-\mathrm{H}(10)$ & 0.9900 & $\mathrm{C}(4)-\mathrm{N}(2)-\mathrm{C}(5)$ & $108.27(15)$ \\
\hline $\mathrm{C}(6)-\mathrm{H}(6 \mathrm{~A})$ & 0.9900 & $\mathrm{C}(4)-\mathrm{N}(2)-\mathrm{C}(2)$ & $110.61(15)$ \\
\hline $\mathrm{C}(6)-\mathrm{H}(6 \mathrm{~B})$ & 0.9900 & $\mathrm{C}(5)-\mathrm{N}(2)-\mathrm{C}(2)$ & $111.15(16)$ \\
\hline$C(6 S)-C(7 S)$ & $1.521(4)$ & $\mathrm{C}(6)-\mathrm{N}(3)-\mathrm{C}(3)$ & $109.00(15)$ \\
\hline $\mathrm{C}(6 \mathrm{~S})-\mathrm{H}(11)$ & 0.9900 & $\mathrm{C}(6)-\mathrm{N}(3)-\mathrm{C}(5)$ & $108.28(15)$ \\
\hline $\mathrm{C}(6 \mathrm{~S})-\mathrm{H}(12)$ & 0.9900 & $C(3)-N(3)-C(5)$ & $110.40(15)$ \\
\hline $\mathrm{C}(7)-\mathrm{C}(8)$ & $1.524(3)$ & $\mathrm{N}(1)-\mathrm{C}(1)-\mathrm{C}(7)$ & $112.75(15)$ \\
\hline$C(7)-C(15)$ & $1.537(3)$ & $\mathrm{N}(1)-\mathrm{C}(1)-\mathrm{P}(1)$ & $106.10(12)$ \\
\hline
\end{tabular}




\begin{tabular}{|c|c|c|c|}
\hline $\mathrm{C}(7)-\mathrm{C}(1)-\mathrm{P}(1)$ & 117.61(13) & $\mathrm{C}(6 \mathrm{~S})-\mathrm{C}(5 \mathrm{~S})-\mathrm{H}(10)$ & 110.4 \\
\hline $\mathrm{N}(1)-\mathrm{C}(1)-\mathrm{H}(1 \mathrm{~B})$ & 106.6 & $\mathrm{H}(9)-\mathrm{C}(5 \mathrm{~S})-\mathrm{H}(10)$ & 108.6 \\
\hline $\mathrm{C}(7)-\mathrm{C}(1)-\mathrm{H}(1 \mathrm{~B})$ & 106.6 & $\mathrm{~N}(1)-\mathrm{C}(6)-\mathrm{N}(3)$ & $114.63(16)$ \\
\hline $\mathrm{P}(1)-\mathrm{C}(1)-\mathrm{H}(1 \mathrm{~B})$ & 106.6 & $\mathrm{~N}(1)-\mathrm{C}(6)-\mathrm{H}(6 \mathrm{~A})$ & 108.6 \\
\hline $\mathrm{O}(1 \mathrm{~S})-\mathrm{C}(1 \mathrm{~S})-\mathrm{C}(2 \mathrm{~S})$ & $105.98(19)$ & $\mathrm{N}(3)-\mathrm{C}(6)-\mathrm{H}(6 \mathrm{~A})$ & 108.6 \\
\hline $\mathrm{O}(1 \mathrm{~S})-\mathrm{C}(1 \mathrm{~S})-\mathrm{H}(1)$ & 110.5 & $\mathrm{~N}(1)-\mathrm{C}(6)-\mathrm{H}(6 \mathrm{~B})$ & 108.6 \\
\hline $\mathrm{C}(2 \mathrm{~S})-\mathrm{C}(1 \mathrm{~S})-\mathrm{H}(1)$ & 110.5 & $\mathrm{~N}(3)-\mathrm{C}(6)-\mathrm{H}(6 \mathrm{~B})$ & 108.6 \\
\hline $\mathrm{O}(1 \mathrm{~S})-\mathrm{C}(1 \mathrm{~S})-\mathrm{H}(2)$ & 110.5 & $\mathrm{H}(6 \mathrm{~A})-\mathrm{C}(6)-\mathrm{H}(6 \mathrm{~B})$ & 107.6 \\
\hline $\mathrm{C}(2 \mathrm{~S})-\mathrm{C}(1 \mathrm{~S})-\mathrm{H}(2)$ & 110.5 & $\mathrm{C}(5 \mathrm{~S})-\mathrm{C}(6 \mathrm{~S})-\mathrm{C}(7 \mathrm{~S})$ & $101.3(2)$ \\
\hline $\mathrm{H}(1)-\mathrm{C}(1 \mathrm{~S})-\mathrm{H}(2)$ & 108.7 & $\mathrm{C}(5 \mathrm{~S})-\mathrm{C}(6 \mathrm{~S})-\mathrm{H}(11)$ & 111.5 \\
\hline $\mathrm{N}(2)-\mathrm{C}(2)-\mathrm{P}(1)$ & $110.41(13)$ & $\mathrm{C}(7 \mathrm{~S})-\mathrm{C}(6 \mathrm{~S})-\mathrm{H}(11)$ & 111.5 \\
\hline $\mathrm{N}(2)-\mathrm{C}(2)-\mathrm{H}(2 \mathrm{~B})$ & 109.6 & $\mathrm{C}(5 \mathrm{~S})-\mathrm{C}(6 \mathrm{~S})-\mathrm{H}(12)$ & 111.5 \\
\hline $\mathrm{P}(1)-\mathrm{C}(2)-\mathrm{H}(2 \mathrm{~B})$ & 109.6 & $\mathrm{C}(7 \mathrm{~S})-\mathrm{C}(6 \mathrm{~S})-\mathrm{H}(12)$ & 111.5 \\
\hline $\mathrm{N}(2)-\mathrm{C}(2)-\mathrm{H}(2 \mathrm{C})$ & 109.6 & $\mathrm{H}(11)-\mathrm{C}(6 \mathrm{~S})-\mathrm{H}(12)$ & 109.3 \\
\hline $\mathrm{P}(1)-\mathrm{C}(2)-\mathrm{H}(2 \mathrm{C})$ & 109.6 & $\mathrm{O}(2)-\mathrm{C}(7)-\mathrm{C}(8)$ & $107.12(15)$ \\
\hline $\mathrm{H}(2 \mathrm{~B})-\mathrm{C}(2)-\mathrm{H}(2 \mathrm{C})$ & 108.1 & $\mathrm{O}(2)-\mathrm{C}(7)-\mathrm{C}(15)$ & $109.37(15)$ \\
\hline$C(1 S)-C(2 S)-C(3 S)$ & $101.5(2)$ & $C(8)-C(7)-C(15)$ & $107.96(15)$ \\
\hline $\mathrm{C}(1 \mathrm{~S})-\mathrm{C}(2 \mathrm{~S})-\mathrm{H}(3)$ & 111.5 & $\mathrm{O}(2)-\mathrm{C}(7)-\mathrm{C}(1)$ & $110.55(15)$ \\
\hline $\mathrm{C}(3 \mathrm{~S})-\mathrm{C}(2 \mathrm{~S})-\mathrm{H}(3)$ & 111.5 & $\mathrm{C}(8)-\mathrm{C}(7)-\mathrm{C}(1)$ & $108.40(15)$ \\
\hline $\mathrm{C}(1 \mathrm{~S})-\mathrm{C}(2 \mathrm{~S})-\mathrm{H}(4)$ & 111.5 & $C(15)-C(7)-C(1)$ & $113.21(15)$ \\
\hline $\mathrm{C}(3 \mathrm{~S})-\mathrm{C}(2 \mathrm{~S})-\mathrm{H}(4)$ & 111.5 & $\mathrm{C}(8 \mathrm{~S})-\mathrm{C}(7 \mathrm{~S})-\mathrm{C}(6 \mathrm{~S})$ & $103.8(2)$ \\
\hline $\mathrm{H}(3)-\mathrm{C}(2 \mathrm{~S})-\mathrm{H}(4)$ & 109.3 & $\mathrm{C}(8 \mathrm{~S})-\mathrm{C}(7 \mathrm{~S})-\mathrm{H}(13)$ & 111.0 \\
\hline $\mathrm{N}(3)-\mathrm{C}(3)-\mathrm{P}(1)$ & $112.25(13)$ & $\mathrm{C}(6 \mathrm{~S})-\mathrm{C}(7 \mathrm{~S})-\mathrm{H}(13)$ & 111.0 \\
\hline $\mathrm{N}(3)-\mathrm{C}(3)-\mathrm{H}(3 \mathrm{~A})$ & 109.2 & $\mathrm{C}(8 \mathrm{~S})-\mathrm{C}(7 \mathrm{~S})-\mathrm{H}(14)$ & 111.0 \\
\hline $\mathrm{P}(1)-\mathrm{C}(3)-\mathrm{H}(3 \mathrm{~A})$ & 109.2 & $\mathrm{C}(6 \mathrm{~S})-\mathrm{C}(7 \mathrm{~S})-\mathrm{H}(14)$ & 111.0 \\
\hline $\mathrm{N}(3)-\mathrm{C}(3)-\mathrm{H}(3 \mathrm{~B})$ & 109.2 & $\mathrm{H}(13)-\mathrm{C}(7 \mathrm{~S})-\mathrm{H}(14)$ & 109.0 \\
\hline $\mathrm{P}(1)-\mathrm{C}(3)-\mathrm{H}(3 \mathrm{~B})$ & 109.2 & $\mathrm{C}(13)-\mathrm{C}(8)-\mathrm{C}(9)$ & $117.57(18)$ \\
\hline $\mathrm{H}(3 \mathrm{~A})-\mathrm{C}(3)-\mathrm{H}(3 \mathrm{~B})$ & 107.9 & $\mathrm{C}(13)-\mathrm{C}(8)-\mathrm{C}(7)$ & $122.56(17)$ \\
\hline $\mathrm{C}(2 \mathrm{~S})-\mathrm{C}(3 \mathrm{~S})-\mathrm{C}(4 \mathrm{~S})$ & $102.3(2)$ & $C(9)-C(8)-C(7)$ & $119.87(17)$ \\
\hline $\mathrm{C}(2 \mathrm{~S})-\mathrm{C}(3 \mathrm{~S})-\mathrm{H}(5)$ & 111.3 & $\mathrm{O}(2 \mathrm{~S})-\mathrm{C}(8 \mathrm{~S})-\mathrm{C}(7 \mathrm{~S})$ & $108.1(2)$ \\
\hline $\mathrm{C}(4 \mathrm{~S})-\mathrm{C}(3 \mathrm{~S})-\mathrm{H}(5)$ & 111.3 & $\mathrm{O}(2 \mathrm{~S})-\mathrm{C}(8 \mathrm{~S})-\mathrm{H}(15)$ & 110.1 \\
\hline $\mathrm{C}(2 \mathrm{~S})-\mathrm{C}(3 \mathrm{~S})-\mathrm{H}(6)$ & 111.3 & $\mathrm{C}(7 \mathrm{~S})-\mathrm{C}(8 \mathrm{~S})-\mathrm{H}(15)$ & 110.1 \\
\hline $\mathrm{C}(4 \mathrm{~S})-\mathrm{C}(3 \mathrm{~S})-\mathrm{H}(6)$ & 111.3 & $\mathrm{O}(2 \mathrm{~S})-\mathrm{C}(8 \mathrm{~S})-\mathrm{H}(16)$ & 110.1 \\
\hline $\mathrm{H}(5)-\mathrm{C}(3 \mathrm{~S})-\mathrm{H}(6)$ & 109.2 & $\mathrm{C}(7 \mathrm{~S})-\mathrm{C}(8 \mathrm{~S})-\mathrm{H}(16)$ & 110.1 \\
\hline $\mathrm{N}(2)-\mathrm{C}(4)-\mathrm{N}(1)$ & 114.41(16) & $\mathrm{H}(15)-\mathrm{C}(8 \mathrm{~S})-\mathrm{H}(16)$ & 108.4 \\
\hline $\mathrm{N}(2)-\mathrm{C}(4)-\mathrm{H}(4 \mathrm{~A})$ & 108.7 & $C(10)-C(9)-C(8)$ & $121.33(18)$ \\
\hline $\mathrm{N}(1)-\mathrm{C}(4)-\mathrm{H}(4 \mathrm{~A})$ & 108.7 & $\mathrm{C}(10)-\mathrm{C}(9)-\mathrm{H}(9 \mathrm{~A})$ & 119.3 \\
\hline $\mathrm{N}(2)-\mathrm{C}(4)-\mathrm{H}(4 \mathrm{~B})$ & 108.7 & $\mathrm{C}(8)-\mathrm{C}(9)-\mathrm{H}(9 \mathrm{~A})$ & 119.3 \\
\hline $\mathrm{N}(1)-\mathrm{C}(4)-\mathrm{H}(4 \mathrm{~B})$ & 108.7 & $C(9)-C(10)-C(11)$ & $120.08(18)$ \\
\hline $\mathrm{H}(4 \mathrm{~A})-\mathrm{C}(4)-\mathrm{H}(4 \mathrm{~B})$ & 107.6 & $\mathrm{C}(9)-\mathrm{C}(10)-\mathrm{H}(10 \mathrm{~A})$ & 120.0 \\
\hline $\mathrm{O}(1 \mathrm{~S})-\mathrm{C}(4 \mathrm{~S})-\mathrm{C}(3 \mathrm{~S})$ & $106.51(19)$ & $\mathrm{C}(11)-\mathrm{C}(10)-\mathrm{H}(10 \mathrm{~A})$ & 120.0 \\
\hline $\mathrm{O}(1 \mathrm{~S})-\mathrm{C}(4 \mathrm{~S})-\mathrm{H}(7)$ & 110.4 & $\mathrm{O}(3)-\mathrm{C}(11)-\mathrm{C}(12)$ & $125.14(18)$ \\
\hline $\mathrm{C}(3 \mathrm{~S})-\mathrm{C}(4 \mathrm{~S})-\mathrm{H}(7)$ & 110.4 & $\mathrm{O}(3)-\mathrm{C}(11)-\mathrm{C}(10)$ & $115.28(17)$ \\
\hline $\mathrm{O}(1 \mathrm{~S})-\mathrm{C}(4 \mathrm{~S})-\mathrm{H}(8)$ & 110.4 & $C(12)-C(11)-C(10)$ & $119.57(19)$ \\
\hline $\mathrm{C}(3 \mathrm{~S})-\mathrm{C}(4 \mathrm{~S})-\mathrm{H}(8)$ & 110.4 & $C(11)-C(12)-C(13)$ & $119.66(18)$ \\
\hline $\mathrm{H}(7)-\mathrm{C}(4 \mathrm{~S})-\mathrm{H}(8)$ & 108.6 & $\mathrm{C}(11)-\mathrm{C}(12)-\mathrm{H}(12 \mathrm{~A})$ & 120.2 \\
\hline $\mathrm{N}(2)-\mathrm{C}(5)-\mathrm{N}(3)$ & 114.81(16) & $\mathrm{C}(13)-\mathrm{C}(12)-\mathrm{H}(12 \mathrm{~A})$ & 120.2 \\
\hline $\mathrm{N}(2)-\mathrm{C}(5)-\mathrm{H}(5 \mathrm{~B})$ & 108.6 & $\mathrm{C}(8)-\mathrm{C}(13)-\mathrm{C}(12)$ & $121.74(18)$ \\
\hline $\mathrm{N}(3)-\mathrm{C}(5)-\mathrm{H}(5 \mathrm{~B})$ & 108.6 & $\mathrm{C}(8)-\mathrm{C}(13)-\mathrm{H}(13 \mathrm{~A})$ & 119.1 \\
\hline $\mathrm{N}(2)-\mathrm{C}(5)-\mathrm{H}(5 \mathrm{C})$ & 108.6 & $\mathrm{C}(12)-\mathrm{C}(13)-\mathrm{H}(13 \mathrm{~A})$ & 119.1 \\
\hline $\mathrm{N}(3)-\mathrm{C}(5)-\mathrm{H}(5 \mathrm{C})$ & 108.6 & $\mathrm{O}(3)-\mathrm{C}(14)-\mathrm{H}(14 \mathrm{~A})$ & 109.5 \\
\hline $\mathrm{H}(5 \mathrm{~B})-\mathrm{C}(5)-\mathrm{H}(5 \mathrm{C})$ & 107.5 & $\mathrm{O}(3)-\mathrm{C}(14)-\mathrm{H}(14 \mathrm{~B})$ & 109.5 \\
\hline $\mathrm{O}(2 \mathrm{~S})-\mathrm{C}(5 \mathrm{~S})-\mathrm{C}(6 \mathrm{~S})$ & $106.7(2)$ & $\mathrm{H}(14 \mathrm{~A})-\mathrm{C}(14)-\mathrm{H}(14 \mathrm{~B})$ & 109.5 \\
\hline $\mathrm{O}(2 \mathrm{~S})-\mathrm{C}(5 \mathrm{~S})-\mathrm{H}(9)$ & 110.4 & $\mathrm{O}(3)-\mathrm{C}(14)-\mathrm{H}(14 \mathrm{C})$ & 109.5 \\
\hline $\mathrm{C}(6 \mathrm{~S})-\mathrm{C}(5 \mathrm{~S})-\mathrm{H}(9)$ & 110.4 & $\mathrm{H}(14 \mathrm{~A})-\mathrm{C}(14)-\mathrm{H}(14 \mathrm{C})$ & 109.5 \\
\hline $\mathrm{O}(2 \mathrm{~S})-\mathrm{C}(5 \mathrm{~S})-\mathrm{H}(10)$ & 110.4 & $\mathrm{H}(14 \mathrm{~B})-\mathrm{C}(14)-\mathrm{H}(14 \mathrm{C})$ & 109.5 \\
\hline
\end{tabular}




$\begin{array}{llll}\mathrm{C}(16)-\mathrm{C}(15)-\mathrm{C}(20) & 117.61(18) & \mathrm{C}(20)-\mathrm{C}(19)-\mathrm{C}(18) & 119.55(18) \\ \mathrm{C}(16)-\mathrm{C}(15)-\mathrm{C}(7) & 125.22(17) & \mathrm{C}(20)-\mathrm{C}(19)-\mathrm{H}(19 \mathrm{~A}) & 120.2 \\ \mathrm{C}(20)-\mathrm{C}(15)-\mathrm{C}(7) & 117.05(17) & \mathrm{C}(18)-\mathrm{C}(19)-\mathrm{H}(19 \mathrm{~A}) & 120.2 \\ \mathrm{C}(15)-\mathrm{C}(16)-\mathrm{C}(17) & 121.25(18) & \mathrm{C}(19)-\mathrm{C}(20)-\mathrm{C}(15) & 121.81(19) \\ \mathrm{C}(15)-\mathrm{C}(16)-\mathrm{H}(16 \mathrm{~A}) & 119.4 & \mathrm{C}(19)-\mathrm{C}(20)-\mathrm{H}(20 \mathrm{~A}) & 119.1 \\ \mathrm{C}(17)-\mathrm{C}(16)-\mathrm{H}(16 \mathrm{~A}) & 119.4 & \mathrm{C}(15)-\mathrm{C}(20)-\mathrm{H}(20 \mathrm{~A}) & 119.1 \\ \mathrm{C}(18)-\mathrm{C}(17)-\mathrm{C}(16) & 120.06(18) & \mathrm{O}(4)-\mathrm{C}(21)-\mathrm{H}(21 \mathrm{~A}) & 109.5 \\ \mathrm{C}(18)-\mathrm{C}(17)-\mathrm{H}(17 \mathrm{~A}) & 120.0 & \mathrm{O}(4)-\mathrm{C}(21)-\mathrm{H}(21 \mathrm{~B}) & 109.5 \\ \mathrm{C}(16)-\mathrm{C}(17)-\mathrm{H}(17 \mathrm{~A}) & 120.0 & \mathrm{H}(21 \mathrm{~A})-\mathrm{C}(21)-\mathrm{H}(21 \mathrm{~B}) & 109.5 \\ \mathrm{O}(4)-\mathrm{C}(18)-\mathrm{C}(17) & 124.31(18) & \mathrm{O}(4)-\mathrm{C}(21)-\mathrm{H}(21 \mathrm{C}) & 109.5 \\ \mathrm{O}(4)-\mathrm{C}(18)-\mathrm{C}(19) & 116.00(18) & \mathrm{H}(21 \mathrm{~A})-\mathrm{C}(21)-\mathrm{H}(21 \mathrm{C}) & 109.5 \\ \mathrm{C}(17)-\mathrm{C}(18)-\mathrm{C}(19) & 119.67(18) & \mathrm{H}(21 \mathrm{~B})-\mathrm{C}(21)-\mathrm{H}(21 \mathrm{C}) & 109.5 \\ & & & \end{array}$

Symmetry transformations used to generate equivalent atoms:

Table 4-S. Hydrogen bonds for $\mathrm{O}=\mathrm{PTA}-\mathrm{C}\left(\mathrm{C}_{6} \mathrm{H}_{4} \mathrm{OCH}_{3}\right)_{2} \mathrm{OH}\left[\AA\right.$ and $\left.^{\circ}\right]$.

\begin{tabular}{lcccc}
\hline D-H...A & d(D-H) & d(H...A) & $d(D \ldots A)$ & $<($ DHA $)$ \\
\hline $\mathrm{O}(2)-H(2 A) \ldots O(1) \# 1$ & 0.84 & 1.85 & $2.6778(19)$ & 168.1 \\
\hline
\end{tabular}

Symmetry transformations used to generate equivalent atoms:

\#1-x,-y,-z

Table 5-S. Bond lengths $[\AA]$ and angles $\left[^{\circ}\right]$ for $\mathrm{O}=\mathrm{PTA}-\mathrm{CH}\left(\mathrm{C}_{6} \mathrm{H}_{4} \mathrm{OCH}_{3}\right) \mathrm{OH}$.

\begin{tabular}{|c|c|c|c|}
\hline $\mathrm{P}(1)-\mathrm{O}(3)$ & $1.478(3)$ & $\mathrm{C}(5)-\mathrm{H}(5 \mathrm{~B})$ & 0.9900 \\
\hline $\mathrm{P}(1)-\mathrm{C}(2)$ & $1.815(4)$ & $\mathrm{C}(6)-\mathrm{H}(6 \mathrm{~A})$ & 0.9900 \\
\hline $\mathrm{P}(1)-\mathrm{C}(3)$ & $1.815(3)$ & $\mathrm{C}(6)-\mathrm{H}(6 \mathrm{~B})$ & 0.9900 \\
\hline $\mathrm{P}(1)-\mathrm{C}(1)$ & $1.832(4)$ & $C(7)-C(8)$ & $1.524(5)$ \\
\hline $\mathrm{O}(1)-\mathrm{C}(7)$ & $1.419(4)$ & $\mathrm{C}(7)-\mathrm{H}(7 \mathrm{~A})$ & 1.0000 \\
\hline $\mathrm{O}(1)-\mathrm{H}(1 \mathrm{~A})$ & 0.8400 & $\mathrm{C}(8)-\mathrm{C}(9)$ & $1.382(5)$ \\
\hline $\mathrm{O}(2)-\mathrm{C}(11)$ & $1.370(4)$ & $\mathrm{C}(8)-\mathrm{C}(13)$ & $1.385(5)$ \\
\hline $\mathrm{O}(2)-\mathrm{C}(14)$ & $1.420(5)$ & $\mathrm{C}(9)-\mathrm{C}(10)$ & $1.377(5)$ \\
\hline $\mathrm{N}(1)-\mathrm{C}(6)$ & $1.477(4)$ & $\mathrm{C}(9)-\mathrm{H}(9 \mathrm{~A})$ & 0.9500 \\
\hline $\mathrm{N}(1)-\mathrm{C}(4)$ & $1.477(4)$ & $\mathrm{C}(10)-\mathrm{C}(11)$ & $1.382(5)$ \\
\hline $\mathrm{N}(1)-\mathrm{C}(1)$ & $1.492(4)$ & $\mathrm{C}(10)-\mathrm{H}(10 \mathrm{~A})$ & 0.9500 \\
\hline $\mathrm{N}(2)-\mathrm{C}(4)$ & $1.463(5)$ & $\mathrm{C}(11)-\mathrm{C}(12)$ & $1.384(5)$ \\
\hline$N(2)-C(5)$ & $1.469(4)$ & $C(12)-C(13)$ & $1.392(5)$ \\
\hline $\mathrm{N}(2)-\mathrm{C}(2)$ & $1.479(4)$ & $\mathrm{C}(12)-\mathrm{H}(12 \mathrm{~A})$ & 0.9500 \\
\hline$N(3)-C(5)$ & $1.464(4)$ & $\mathrm{C}(13)-\mathrm{H}(13 \mathrm{~A})$ & 0.9500 \\
\hline $\mathrm{N}(3)-\mathrm{C}(6)$ & $1.465(5)$ & $\mathrm{C}(14)-\mathrm{H}(14 \mathrm{~A})$ & 0.9800 \\
\hline $\mathrm{N}(3)-\mathrm{C}(3)$ & $1.485(5)$ & $\mathrm{C}(14)-\mathrm{H}(14 \mathrm{~B})$ & 0.9800 \\
\hline$C(1)-C(7)$ & $1.536(5)$ & $\mathrm{C}(14)-\mathrm{H}(14 \mathrm{C})$ & 0.9800 \\
\hline $\mathrm{C}(1)-\mathrm{H}(1 \mathrm{~B})$ & 1.0000 & & \\
\hline $\mathrm{C}(2)-\mathrm{H}(2 \mathrm{~A})$ & 0.9900 & $\mathrm{O}(3)-\mathrm{P}(1)-\mathrm{C}(2)$ & $115.22(16)$ \\
\hline $\mathrm{C}(2)-\mathrm{H}(2 \mathrm{~B})$ & 0.9900 & $\mathrm{O}(3)-\mathrm{P}(1)-\mathrm{C}(3)$ & $118.09(17)$ \\
\hline $\mathrm{C}(3)-\mathrm{H}(3 \mathrm{~B})$ & 0.9900 & $\mathrm{C}(2)-\mathrm{P}(1)-\mathrm{C}(3)$ & $99.74(17)$ \\
\hline $\mathrm{C}(3)-\mathrm{H}(3 \mathrm{C})$ & 0.9900 & $\mathrm{O}(3)-\mathrm{P}(1)-\mathrm{C}(1)$ & $118.31(15)$ \\
\hline $\mathrm{C}(4)-\mathrm{H}(4 \mathrm{~A})$ & 0.9900 & $\mathrm{C}(2)-\mathrm{P}(1)-\mathrm{C}(1)$ & $99.43(17)$ \\
\hline $\mathrm{C}(4)-\mathrm{H}(4 \mathrm{~B})$ & 0.9900 & $\mathrm{C}(3)-\mathrm{P}(1)-\mathrm{C}(1)$ & $102.88(16)$ \\
\hline $\mathrm{C}(5)-\mathrm{H}(5 \mathrm{~A})$ & 0.9900 & $\mathrm{C}(7)-\mathrm{O}(1)-\mathrm{H}(1 \mathrm{~A})$ & 109.5 \\
\hline
\end{tabular}




\begin{tabular}{|c|c|c|c|}
\hline $\mathrm{C}(11)-\mathrm{O}(2)-\mathrm{C}(14)$ & $118.5(4)$ & $\mathrm{N}(2)-\mathrm{C}(5)-\mathrm{H}(5 \mathrm{~B})$ & 108.5 \\
\hline $\mathrm{C}(6)-\mathrm{N}(1)-\mathrm{C}(4)$ & $107.4(3)$ & $\mathrm{H}(5 \mathrm{~A})-\mathrm{C}(5)-\mathrm{H}(5 \mathrm{~B})$ & 107.5 \\
\hline$C(6)-N(1)-C(1)$ & 113.1(3) & $\mathrm{N}(3)-\mathrm{C}(6)-\mathrm{N}(1)$ & $114.5(3)$ \\
\hline $\mathrm{C}(4)-\mathrm{N}(1)-\mathrm{C}(1)$ & $111.3(3)$ & $\mathrm{N}(3)-\mathrm{C}(6)-\mathrm{H}(6 \mathrm{~A})$ & 108.6 \\
\hline $\mathrm{C}(4)-\mathrm{N}(2)-\mathrm{C}(5)$ & $108.0(3)$ & $\mathrm{N}(1)-\mathrm{C}(6)-\mathrm{H}(6 \mathrm{~A})$ & 108.6 \\
\hline $\mathrm{C}(4)-\mathrm{N}(2)-\mathrm{C}(2)$ & $109.9(3)$ & $\mathrm{N}(3)-\mathrm{C}(6)-\mathrm{H}(6 \mathrm{~B})$ & 108.6 \\
\hline $\mathrm{C}(5)-\mathrm{N}(2)-\mathrm{C}(2)$ & $110.3(3)$ & $\mathrm{N}(1)-\mathrm{C}(6)-\mathrm{H}(6 \mathrm{~B})$ & 108.6 \\
\hline $\mathrm{C}(5)-\mathrm{N}(3)-\mathrm{C}(6)$ & $108.7(3)$ & $\mathrm{H}(6 \mathrm{~A})-\mathrm{C}(6)-\mathrm{H}(6 \mathrm{~B})$ & 107.6 \\
\hline $\mathrm{C}(5)-\mathrm{N}(3)-\mathrm{C}(3)$ & $111.5(3)$ & $\mathrm{O}(1)-\mathrm{C}(7)-\mathrm{C}(8)$ & $111.4(3)$ \\
\hline$C(6)-N(3)-C(3)$ & $110.8(3)$ & $\mathrm{O}(1)-\mathrm{C}(7)-\mathrm{C}(1)$ & $108.9(3)$ \\
\hline $\mathrm{N}(1)-\mathrm{C}(1)-\mathrm{C}(7)$ & $111.4(3)$ & $C(8)-C(7)-C(1)$ & $112.8(3)$ \\
\hline $\mathrm{N}(1)-\mathrm{C}(1)-\mathrm{P}(1)$ & $107.9(2)$ & $\mathrm{O}(1)-\mathrm{C}(7)-\mathrm{H}(7 \mathrm{~A})$ & 107.9 \\
\hline $\mathrm{C}(7)-\mathrm{C}(1)-\mathrm{P}(1)$ & $117.5(3)$ & $\mathrm{C}(8)-\mathrm{C}(7)-\mathrm{H}(7 \mathrm{~A})$ & 107.9 \\
\hline $\mathrm{N}(1)-\mathrm{C}(1)-\mathrm{H}(1 \mathrm{~B})$ & 106.5 & $\mathrm{C}(1)-\mathrm{C}(7)-\mathrm{H}(7 \mathrm{~A})$ & 107.9 \\
\hline $\mathrm{C}(7)-\mathrm{C}(1)-\mathrm{H}(1 \mathrm{~B})$ & 106.5 & $\mathrm{C}(9)-\mathrm{C}(8)-\mathrm{C}(13)$ & $118.8(3)$ \\
\hline $\mathrm{P}(1)-\mathrm{C}(1)-\mathrm{H}(1 \mathrm{~B})$ & 106.5 & $\mathrm{C}(9)-\mathrm{C}(8)-\mathrm{C}(7)$ & 122.1(3) \\
\hline $\mathrm{N}(2)-\mathrm{C}(2)-\mathrm{P}(1)$ & $112.1(2)$ & $\mathrm{C}(13)-\mathrm{C}(8)-\mathrm{C}(7)$ & 119.1(3) \\
\hline $\mathrm{N}(2)-\mathrm{C}(2)-\mathrm{H}(2 \mathrm{~A})$ & 109.2 & $\mathrm{C}(10)-\mathrm{C}(9)-\mathrm{C}(8)$ & $120.6(3)$ \\
\hline $\mathrm{P}(1)-\mathrm{C}(2)-\mathrm{H}(2 \mathrm{~A})$ & 109.2 & $\mathrm{C}(10)-\mathrm{C}(9)-\mathrm{H}(9 \mathrm{~A})$ & 119.7 \\
\hline $\mathrm{N}(2)-\mathrm{C}(2)-\mathrm{H}(2 \mathrm{~B})$ & 109.2 & $\mathrm{C}(8)-\mathrm{C}(9)-\mathrm{H}(9 \mathrm{~A})$ & 119.7 \\
\hline $\mathrm{P}(1)-\mathrm{C}(2)-\mathrm{H}(2 \mathrm{~B})$ & 109.2 & $C(9)-C(10)-C(11)$ & $120.4(4)$ \\
\hline $\mathrm{H}(2 \mathrm{~A})-\mathrm{C}(2)-\mathrm{H}(2 \mathrm{~B})$ & 107.9 & $\mathrm{C}(9)-\mathrm{C}(10)-\mathrm{H}(10 \mathrm{~A})$ & 119.8 \\
\hline $\mathrm{N}(3)-\mathrm{C}(3)-\mathrm{P}(1)$ & $109.8(2)$ & $\mathrm{C}(11)-\mathrm{C}(10)-\mathrm{H}(10 \mathrm{~A})$ & 119.8 \\
\hline $\mathrm{N}(3)-\mathrm{C}(3)-\mathrm{H}(3 \mathrm{~B})$ & 109.7 & $\mathrm{O}(2)-\mathrm{C}(11)-\mathrm{C}(10)$ & $116.2(4)$ \\
\hline $\mathrm{P}(1)-\mathrm{C}(3)-\mathrm{H}(3 \mathrm{~B})$ & 109.7 & $\mathrm{O}(2)-\mathrm{C}(11)-\mathrm{C}(12)$ & $123.9(4)$ \\
\hline $\mathrm{N}(3)-\mathrm{C}(3)-\mathrm{H}(3 \mathrm{C})$ & 109.7 & $C(10)-C(11)-C(12)$ & $119.9(3)$ \\
\hline $\mathrm{P}(1)-\mathrm{C}(3)-\mathrm{H}(3 \mathrm{C})$ & 109.7 & $\mathrm{C}(11)-\mathrm{C}(12)-\mathrm{C}(13)$ & 119.1(3) \\
\hline $\mathrm{H}(3 \mathrm{~B})-\mathrm{C}(3)-\mathrm{H}(3 \mathrm{C})$ & 108.2 & $\mathrm{C}(11)-\mathrm{C}(12)-\mathrm{H}(12 \mathrm{~A})$ & 120.5 \\
\hline $\mathrm{N}(2)-\mathrm{C}(4)-\mathrm{N}(1)$ & $115.1(3)$ & $\mathrm{C}(13)-\mathrm{C}(12)-\mathrm{H}(12 \mathrm{~A})$ & 120.5 \\
\hline $\mathrm{N}(2)-\mathrm{C}(4)-\mathrm{H}(4 \mathrm{~A})$ & 108.5 & $C(8)-C(13)-C(12)$ & 121.1(4) \\
\hline $\mathrm{N}(1)-\mathrm{C}(4)-\mathrm{H}(4 \mathrm{~A})$ & 108.5 & $\mathrm{C}(8)-\mathrm{C}(13)-\mathrm{H}(13 \mathrm{~A})$ & 119.4 \\
\hline $\mathrm{N}(2)-\mathrm{C}(4)-\mathrm{H}(4 \mathrm{~B})$ & 108.5 & $\mathrm{C}(12)-\mathrm{C}(13)-\mathrm{H}(13 \mathrm{~A})$ & 119.4 \\
\hline $\mathrm{N}(1)-\mathrm{C}(4)-\mathrm{H}(4 \mathrm{~B})$ & 108.5 & $\mathrm{O}(2)-\mathrm{C}(14)-\mathrm{H}(14 \mathrm{~A})$ & 109.5 \\
\hline $\mathrm{H}(4 \mathrm{~A})-\mathrm{C}(4)-\mathrm{H}(4 \mathrm{~B})$ & 107.5 & $\mathrm{O}(2)-\mathrm{C}(14)-\mathrm{H}(14 \mathrm{~B})$ & 109.5 \\
\hline $\mathrm{N}(3)-\mathrm{C}(5)-\mathrm{N}(2)$ & $114.9(3)$ & $\mathrm{H}(14 \mathrm{~A})-\mathrm{C}(14)-\mathrm{H}(14 \mathrm{~B})$ & 109.5 \\
\hline $\mathrm{N}(3)-\mathrm{C}(5)-\mathrm{H}(5 \mathrm{~A})$ & 108.5 & $\mathrm{O}(2)-\mathrm{C}(14)-\mathrm{H}(14 \mathrm{C})$ & 109.5 \\
\hline $\mathrm{N}(2)-\mathrm{C}(5)-\mathrm{H}(5 \mathrm{~A})$ & 108.5 & $\mathrm{H}(14 \mathrm{~A})-\mathrm{C}(14)-\mathrm{H}(14 \mathrm{C})$ & 109.5 \\
\hline $\mathrm{N}(3)-\mathrm{C}(5)-\mathrm{H}(5 \mathrm{~B})$ & 108.5 & $\mathrm{H}(14 \mathrm{~B})-\mathrm{C}(14)-\mathrm{H}(14 \mathrm{C})$ & 109.5 \\
\hline
\end{tabular}

Symmetry transformations used to generate equivalent atoms:

Table 6-S. Hydrogen bonds for $\mathrm{O}=\mathrm{PTA}-\mathrm{CH}\left(\mathrm{C}_{6} \mathrm{H}_{4} \mathrm{OCH}_{3}\right) \mathrm{OH}\left[\AA\right.$ and $\left.^{\circ}\right]$.

\begin{tabular}{lcccc}
\hline $\mathrm{D}-\mathrm{H} \ldots \mathrm{A}$ & $\mathrm{d}(\mathrm{D}-\mathrm{H})$ & $\mathrm{d}(\mathrm{H} \ldots \mathrm{A})$ & $\mathrm{d}(\mathrm{D} \ldots \mathrm{A})$ & $<(\mathrm{DHA})$ \\
\hline $\mathrm{O}(1)-\mathrm{H}(1 \mathrm{~A}) \ldots \mathrm{O}(3) \# 1$ & 0.84 & 1.84 & $2.682(4)$ & 177.4
\end{tabular}

Symmetry transformations used to generate equivalent atoms:

$\# 1-x+1, y+1 / 2,-z+1 / 2$ 
Table 7-S. Bond lengths $[\AA ̊]$ and angles $\left[{ }^{\circ}\right]$ for PTA-CH(ferrocenyl)OH.

\begin{tabular}{|c|c|c|c|}
\hline $\mathrm{Fe}(1)-\mathrm{C}(15)$ & $2.026(6)$ & $\mathrm{C}(16)-\mathrm{C}(17)$ & $1.452(9)$ \\
\hline $\mathrm{Fe}(1)-\mathrm{C}(12)$ & $2.029(6)$ & $\mathrm{C}(16)-\mathrm{H}(16 \mathrm{~A})$ & 1.0000 \\
\hline $\mathrm{Fe}(1)-\mathrm{C}(17)$ & $2.034(6)$ & $\mathrm{C}(17)-\mathrm{H}(17 \mathrm{~A})$ & 1.0000 \\
\hline $\mathrm{Fe}(1)-\mathrm{C}(14)$ & $2.031(6)$ & & \\
\hline $\mathrm{Fe}(1)-\mathrm{C}(10)$ & $2.036(6)$ & $\mathrm{C}(15)-\mathrm{Fe}(1)-\mathrm{C}(12)$ & $153.1(3)$ \\
\hline $\mathrm{Fe}(1)-\mathrm{C}(8)$ & $2.037(6)$ & $\mathrm{C}(15)-\mathrm{Fe}(1)-\mathrm{C}(17)$ & $69.0(3)$ \\
\hline $\mathrm{Fe}(1)-\mathrm{C}(16)$ & $2.041(6)$ & $\mathrm{C}(12)-\mathrm{Fe}(1)-\mathrm{C}(17)$ & $107.0(3)$ \\
\hline $\mathrm{Fe}(1)-\mathrm{C}(13)$ & $2.039(5)$ & $\mathrm{C}(15)-\mathrm{Fe}(1)-\mathrm{C}(14)$ & $40.3(2)$ \\
\hline $\mathrm{Fe}(1)-\mathrm{C}(11)$ & $2.041(6)$ & $\mathrm{C}(12)-\mathrm{Fe}(1)-\mathrm{C}(14)$ & $165.1(3)$ \\
\hline $\mathrm{Fe}(1)-\mathrm{C}(9)$ & $2.043(7)$ & $\mathrm{C}(17)-\mathrm{Fe}(1)-\mathrm{C}(14)$ & $68.8(3)$ \\
\hline $\mathrm{P}(1)-\mathrm{C}(3)$ & $1.868(7)$ & $\mathrm{C}(15)-\mathrm{Fe}(1)-\mathrm{C}(10)$ & $107.2(3)$ \\
\hline $\mathrm{P}(1)-\mathrm{C}(1)$ & $1.867(7)$ & $\mathrm{C}(12)-\mathrm{Fe}(1)-\mathrm{C}(10)$ & 68.1(3) \\
\hline $\mathrm{P}(1)-\mathrm{C}(2)$ & $1.867(7)$ & $\mathrm{C}(17)-\mathrm{Fe}(1)-\mathrm{C}(10)$ & $162.1(3)$ \\
\hline $\mathrm{O}(1)-\mathrm{C}(7)$ & $1.443(7)$ & $\mathrm{C}(14)-\mathrm{Fe}(1)-\mathrm{C}(10)$ & $120.3(3)$ \\
\hline $\mathrm{O}(1)-\mathrm{H}(1 \mathrm{~A})$ & $1.03(7)$ & $\mathrm{C}(15)-\mathrm{Fe}(1)-\mathrm{C}(8)$ & $164.3(3)$ \\
\hline $\mathrm{N}(1)-\mathrm{C}(4)$ & $1.458(8)$ & $\mathrm{C}(12)-\mathrm{Fe}(1)-\mathrm{C}(8)$ & $40.5(3)$ \\
\hline $\mathrm{N}(1)-\mathrm{C}(6)$ & $1.472(8)$ & $\mathrm{C}(17)-\mathrm{Fe}(1)-\mathrm{C}(8)$ & $120.7(3)$ \\
\hline $\mathrm{N}(1)-\mathrm{C}(1)$ & $1.469(8)$ & $\mathrm{C}(14)-\mathrm{Fe}(1)-\mathrm{C}(8)$ & $128.3(3)$ \\
\hline $\mathrm{N}(2)-\mathrm{C}(4)$ & $1.451(8)$ & $\mathrm{C}(10)-\mathrm{Fe}(1)-\mathrm{C}(8)$ & $67.5(3)$ \\
\hline $\mathrm{N}(2)-\mathrm{C}(2)$ & $1.452(8)$ & $\mathrm{C}(15)-\mathrm{Fe}(1)-\mathrm{C}(16)$ & $40.3(2)$ \\
\hline $\mathrm{N}(2)-\mathrm{C}(5)$ & $1.468(8)$ & $\mathrm{C}(12)-\mathrm{Fe}(1)-\mathrm{C}(16)$ & $118.8(3)$ \\
\hline $\mathrm{N}(3)-\mathrm{C}(3)$ & $1.450(8)$ & $\mathrm{C}(17)-\mathrm{Fe}(1)-\mathrm{C}(16)$ & $41.7(3)$ \\
\hline $\mathrm{N}(3)-\mathrm{C}(5)$ & $1.465(9)$ & $\mathrm{C}(14)-\mathrm{Fe}(1)-\mathrm{C}(16)$ & $68.2(3)$ \\
\hline $\mathrm{N}(3)-\mathrm{C}(6)$ & $1.473(8)$ & $\mathrm{C}(10)-\mathrm{Fe}(1)-\mathrm{C}(16)$ & $124.2(3)$ \\
\hline$C(1)-C(7)$ & $1.555(8)$ & $\mathrm{C}(8)-\mathrm{Fe}(1)-\mathrm{C}(16)$ & $154.9(3)$ \\
\hline $\mathrm{C}(1)-\mathrm{H}(1 \mathrm{~B})$ & 1.0000 & $\mathrm{C}(15)-\mathrm{Fe}(1)-\mathrm{C}(13)$ & $68.4(3)$ \\
\hline $\mathrm{C}(2)-\mathrm{H}(2 \mathrm{~A})$ & 0.9900 & $\mathrm{C}(12)-\mathrm{Fe}(1)-\mathrm{C}(13)$ & $126.7(3)$ \\
\hline $\mathrm{C}(2)-\mathrm{H}(2 \mathrm{~B})$ & 0.9900 & $\mathrm{C}(17)-\mathrm{Fe}(1)-\mathrm{C}(13)$ & $40.5(2)$ \\
\hline $\mathrm{C}(3)-\mathrm{H}(3 \mathrm{~A})$ & 0.9900 & $\mathrm{C}(14)-\mathrm{Fe}(1)-\mathrm{C}(13)$ & $41.0(3)$ \\
\hline $\mathrm{C}(3)-\mathrm{H}(3 \mathrm{~B})$ & 0.9900 & $\mathrm{C}(10)-\mathrm{Fe}(1)-\mathrm{C}(13)$ & $155.9(3)$ \\
\hline $\mathrm{C}(4)-\mathrm{H}(4 \mathrm{~A})$ & 0.9900 & $\mathrm{C}(8)-\mathrm{Fe}(1)-\mathrm{C}(13)$ & $110.0(3)$ \\
\hline $\mathrm{C}(4)-\mathrm{H}(4 \mathrm{~B})$ & 0.9900 & $\mathrm{C}(16)-\mathrm{Fe}(1)-\mathrm{C}(13)$ & $68.7(2)$ \\
\hline $\mathrm{C}(5)-\mathrm{H}(5 \mathrm{~A})$ & 0.9900 & $\mathrm{C}(15)-\mathrm{Fe}(1)-\mathrm{C}(11)$ & 118.1(3) \\
\hline $\mathrm{C}(5)-\mathrm{H}(5 \mathrm{~B})$ & 0.9900 & $\mathrm{C}(12)-\mathrm{Fe}(1)-\mathrm{C}(11)$ & $41.0(2)$ \\
\hline $\mathrm{C}(6)-\mathrm{H}(6 \mathrm{~A})$ & 0.9900 & $\mathrm{C}(17)-\mathrm{Fe}(1)-\mathrm{C}(11)$ & $124.8(3)$ \\
\hline $\mathrm{C}(6)-\mathrm{H}(6 \mathrm{~B})$ & 0.9900 & $\mathrm{C}(14)-\mathrm{Fe}(1)-\mathrm{C}(11)$ & $153.2(3)$ \\
\hline$C(7)-C(8)$ & $1.505(9)$ & $\mathrm{C}(10)-\mathrm{Fe}(1)-\mathrm{C}(11)$ & 40.3(2) \\
\hline $\mathrm{C}(7)-\mathrm{H}(7 \mathrm{~A})$ & 1.0000 & $\mathrm{C}(8)-\mathrm{Fe}(1)-\mathrm{C}(11)$ & 68.2(3) \\
\hline $\mathrm{C}(8)-\mathrm{C}(12)$ & $1.406(9)$ & $\mathrm{C}(16)-\mathrm{Fe}(1)-\mathrm{C}(11)$ & $105.4(3)$ \\
\hline $\mathrm{C}(8)-\mathrm{C}(9)$ & $1.420(8)$ & $\mathrm{C}(13)-\mathrm{Fe}(1)-\mathrm{C}(11)$ & $163.2(3)$ \\
\hline$C(9)-C(10)$ & $1.405(8)$ & $\mathrm{C}(15)-\mathrm{Fe}(1)-\mathrm{C}(9)$ & $125.8(3)$ \\
\hline $\mathrm{C}(9)-\mathrm{H}(9 \mathrm{~A})$ & 1.0000 & $\mathrm{C}(12)-\mathrm{Fe}(1)-\mathrm{C}(9)$ & $68.8(2)$ \\
\hline$C(10)-C(11)$ & $1.405(9)$ & $\mathrm{C}(17)-\mathrm{Fe}(1)-\mathrm{C}(9)$ & $155.8(3)$ \\
\hline $\mathrm{C}(10)-\mathrm{H}(10 \mathrm{~A})$ & 1.0000 & $\mathrm{C}(14)-\mathrm{Fe}(1)-\mathrm{C}(9)$ & $108.9(3)$ \\
\hline $\mathrm{C}(11)-\mathrm{C}(12)$ & $1.427(8)$ & $\mathrm{C}(10)-\mathrm{Fe}(1)-\mathrm{C}(9)$ & $40.3(2)$ \\
\hline $\mathrm{C}(11)-\mathrm{H}(11 \mathrm{~A})$ & 1.0000 & $\mathrm{C}(8)-\mathrm{Fe}(1)-\mathrm{C}(9)$ & $40.7(2)$ \\
\hline $\mathrm{C}(12)-\mathrm{H}(12 \mathrm{~A})$ & 1.0000 & $\mathrm{C}(16)-\mathrm{Fe}(1)-\mathrm{C}(9)$ & $161.6(2)$ \\
\hline $\mathrm{C}(13)-\mathrm{C}(17)$ & $1.410(8)$ & $\mathrm{C}(13)-\mathrm{Fe}(1)-\mathrm{C}(9)$ & $121.8(3)$ \\
\hline C(13)-C(14) & $1.425(9)$ & $\mathrm{C}(11)-\mathrm{Fe}(1)-\mathrm{C}(9)$ & $68.6(3)$ \\
\hline $\mathrm{C}(13)-\mathrm{H}(13 \mathrm{~A})$ & 1.0000 & $\mathrm{C}(3)-\mathrm{P}(1)-\mathrm{C}(1)$ & $94.0(3)$ \\
\hline $\mathrm{C}(14)-\mathrm{C}(15)$ & $1.397(8)$ & $\mathrm{C}(3)-\mathrm{P}(1)-\mathrm{C}(2)$ & $94.3(3)$ \\
\hline $\mathrm{C}(14)-\mathrm{H}(14 \mathrm{~A})$ & 1.0000 & $\mathrm{C}(1)-\mathrm{P}(1)-\mathrm{C}(2)$ & $96.8(3)$ \\
\hline$C(15)-C(16)$ & $1.403(9)$ & $\mathrm{C}(7)-\mathrm{O}(1)-\mathrm{H}(1 \mathrm{~A})$ & $105(4)$ \\
\hline $\mathrm{C}(15)-\mathrm{H}(15 \mathrm{~A})$ & 1.0000 & $\mathrm{C}(4)-\mathrm{N}(1)-\mathrm{C}(6)$ & 107.1(5) \\
\hline
\end{tabular}




\begin{tabular}{|c|c|c|c|}
\hline $\mathrm{C}(4)-\mathrm{N}(1)-\mathrm{C}(1)$ & $113.2(5)$ & $\mathrm{C}(7)-\mathrm{C}(8)-\mathrm{Fe}(1)$ & $130.5(5)$ \\
\hline $\mathrm{C}(6)-\mathrm{N}(1)-\mathrm{C}(1)$ & $110.2(5)$ & $\mathrm{C}(10)-\mathrm{C}(9)-\mathrm{C}(8)$ & $106.5(6)$ \\
\hline $\mathrm{C}(4)-\mathrm{N}(2)-\mathrm{C}(2)$ & $111.3(6)$ & $\mathrm{C}(10)-\mathrm{C}(9)-\mathrm{Fe}(1)$ & $69.6(4)$ \\
\hline $\mathrm{C}(4)-\mathrm{N}(2)-\mathrm{C}(5)$ & 107.1(5) & $\mathrm{C}(8)-\mathrm{C}(9)-\mathrm{Fe}(1)$ & $69.4(4)$ \\
\hline $\mathrm{C}(2)-\mathrm{N}(2)-\mathrm{C}(5)$ & $111.5(6)$ & $\mathrm{C}(10)-\mathrm{C}(9)-\mathrm{H}(9 \mathrm{~A})$ & 126.7 \\
\hline $\mathrm{C}(3)-\mathrm{N}(3)-\mathrm{C}(5)$ & $111.3(5)$ & $\mathrm{C}(8)-\mathrm{C}(9)-\mathrm{H}(9 \mathrm{~A})$ & 126.7 \\
\hline $\mathrm{C}(3)-\mathrm{N}(3)-\mathrm{C}(6)$ & $111.6(5)$ & $\mathrm{Fe}(1)-\mathrm{C}(9)-\mathrm{H}(9 \mathrm{~A})$ & 126.7 \\
\hline $\mathrm{C}(5)-\mathrm{N}(3)-\mathrm{C}(6)$ & $106.9(6)$ & $\mathrm{C}(9)-\mathrm{C}(10)-\mathrm{C}(11)$ & $109.9(6)$ \\
\hline $\mathrm{N}(1)-\mathrm{C}(1)-\mathrm{C}(7)$ & 109.3(5) & $\mathrm{C}(9)-\mathrm{C}(10)-\mathrm{Fe}(1)$ & $70.1(4)$ \\
\hline $\mathrm{N}(1)-\mathrm{C}(1)-\mathrm{P}(1)$ & $114.0(4)$ & $\mathrm{C}(11)-\mathrm{C}(10)-\mathrm{Fe}(1)$ & $70.0(4)$ \\
\hline$C(7)-C(1)-P(1)$ & $115.4(5)$ & $\mathrm{C}(9)-\mathrm{C}(10)-\mathrm{H}(10 \mathrm{~A})$ & 125.0 \\
\hline $\mathrm{N}(1)-\mathrm{C}(1)-\mathrm{H}(1 \mathrm{~B})$ & 105.7 & $\mathrm{C}(11)-\mathrm{C}(10)-\mathrm{H}(10 \mathrm{~A})$ & 125.0 \\
\hline $\mathrm{C}(7)-\mathrm{C}(1)-\mathrm{H}(1 \mathrm{~B})$ & 105.7 & $\mathrm{Fe}(1)-\mathrm{C}(10)-\mathrm{H}(10 \mathrm{~A})$ & 125.0 \\
\hline $\mathrm{P}(1)-\mathrm{C}(1)-\mathrm{H}(1 \mathrm{~B})$ & 105.7 & $\mathrm{C}(10)-\mathrm{C}(11)-\mathrm{C}(12)$ & $107.0(6)$ \\
\hline $\mathrm{N}(2)-\mathrm{C}(2)-\mathrm{P}(1)$ & $115.1(5)$ & $\mathrm{C}(10)-\mathrm{C}(11)-\mathrm{Fe}(1)$ & $69.6(4)$ \\
\hline $\mathrm{N}(2)-\mathrm{C}(2)-\mathrm{H}(2 \mathrm{~A})$ & 108.5 & $\mathrm{C}(12)-\mathrm{C}(11)-\mathrm{Fe}(1)$ & $69.0(4)$ \\
\hline $\mathrm{P}(1)-\mathrm{C}(2)-\mathrm{H}(2 \mathrm{~A})$ & 108.5 & $\mathrm{C}(10)-\mathrm{C}(11)-\mathrm{H}(11 \mathrm{~A})$ & 126.5 \\
\hline $\mathrm{N}(2)-\mathrm{C}(2)-\mathrm{H}(2 \mathrm{~B})$ & 108.5 & $\mathrm{C}(12)-\mathrm{C}(11)-\mathrm{H}(11 \mathrm{~A})$ & 126.5 \\
\hline $\mathrm{P}(1)-\mathrm{C}(2)-\mathrm{H}(2 \mathrm{~B})$ & 108.5 & $\mathrm{Fe}(1)-\mathrm{C}(11)-\mathrm{H}(11 \mathrm{~A})$ & 126.5 \\
\hline $\mathrm{H}(2 \mathrm{~A})-\mathrm{C}(2)-\mathrm{H}(2 \mathrm{~B})$ & 107.5 & $\mathrm{C}(8)-\mathrm{C}(12)-\mathrm{C}(11)$ & $107.6(6)$ \\
\hline $\mathrm{N}(3)-\mathrm{C}(3)-\mathrm{P}(1)$ & $115.7(4)$ & $\mathrm{C}(8)-\mathrm{C}(12)-\mathrm{Fe}(1)$ & $70.1(4)$ \\
\hline $\mathrm{N}(3)-\mathrm{C}(3)-\mathrm{H}(3 \mathrm{~A})$ & 108.3 & $\mathrm{C}(11)-\mathrm{C}(12)-\mathrm{Fe}(1)$ & $69.9(4)$ \\
\hline $\mathrm{P}(1)-\mathrm{C}(3)-\mathrm{H}(3 \mathrm{~A})$ & 108.3 & $\mathrm{C}(8)-\mathrm{C}(12)-\mathrm{H}(12 \mathrm{~A})$ & 126.2 \\
\hline N(3)-C(3)-H(3B) & 108.3 & $\mathrm{C}(11)-\mathrm{C}(12)-\mathrm{H}(12 \mathrm{~A})$ & 126.2 \\
\hline $\mathrm{P}(1)-\mathrm{C}(3)-\mathrm{H}(3 \mathrm{~B})$ & 108.3 & $\mathrm{Fe}(1)-\mathrm{C}(12)-\mathrm{H}(12 \mathrm{~A})$ & 126.2 \\
\hline $\mathrm{H}(3 \mathrm{~A})-\mathrm{C}(3)-\mathrm{H}(3 \mathrm{~B})$ & 107.4 & $\mathrm{C}(17)-\mathrm{C}(13)-\mathrm{C}(14)$ & $108.1(6)$ \\
\hline $\mathrm{N}(2)-\mathrm{C}(4)-\mathrm{N}(1)$ & $115.4(6)$ & $\mathrm{C}(17)-\mathrm{C}(13)-\mathrm{Fe}(1)$ & $69.5(3)$ \\
\hline $\mathrm{N}(2)-\mathrm{C}(4)-\mathrm{H}(4 \mathrm{~A})$ & 108.4 & $\mathrm{C}(14)-\mathrm{C}(13)-\mathrm{Fe}(1)$ & $69.2(4)$ \\
\hline $\mathrm{N}(1)-\mathrm{C}(4)-\mathrm{H}(4 \mathrm{~A})$ & 108.4 & $\mathrm{C}(17)-\mathrm{C}(13)-\mathrm{H}(13 \mathrm{~A})$ & 125.9 \\
\hline $\mathrm{N}(2)-\mathrm{C}(4)-\mathrm{H}(4 \mathrm{~B})$ & 108.4 & $\mathrm{C}(14)-\mathrm{C}(13)-\mathrm{H}(13 \mathrm{~A})$ & 125.9 \\
\hline $\mathrm{N}(1)-\mathrm{C}(4)-\mathrm{H}(4 \mathrm{~B})$ & 108.4 & $\mathrm{Fe}(1)-\mathrm{C}(13)-\mathrm{H}(13 \mathrm{~A})$ & 125.9 \\
\hline $\mathrm{H}(4 \mathrm{~A})-\mathrm{C}(4)-\mathrm{H}(4 \mathrm{~B})$ & 107.5 & $\mathrm{C}(15)-\mathrm{C}(14)-\mathrm{C}(13)$ & $108.1(6)$ \\
\hline $\mathrm{N}(3)-\mathrm{C}(5)-\mathrm{N}(2)$ & $114.3(6)$ & $\mathrm{C}(15)-\mathrm{C}(14)-\mathrm{Fe}(1)$ & $69.7(4)$ \\
\hline $\mathrm{N}(3)-\mathrm{C}(5)-\mathrm{H}(5 \mathrm{~A})$ & 108.7 & $\mathrm{C}(13)-\mathrm{C}(14)-\mathrm{Fe}(1)$ & $69.8(3)$ \\
\hline $\mathrm{N}(2)-\mathrm{C}(5)-\mathrm{H}(5 \mathrm{~A})$ & 108.7 & $\mathrm{C}(15)-\mathrm{C}(14)-\mathrm{H}(14 \mathrm{~A})$ & 125.9 \\
\hline N(3)-C(5)-H(5B) & 108.7 & $\mathrm{C}(13)-\mathrm{C}(14)-\mathrm{H}(14 \mathrm{~A})$ & 125.9 \\
\hline $\mathrm{N}(2)-\mathrm{C}(5)-\mathrm{H}(5 \mathrm{~B})$ & 108.7 & $\mathrm{Fe}(1)-\mathrm{C}(14)-\mathrm{H}(14 \mathrm{~A})$ & 125.9 \\
\hline $\mathrm{H}(5 \mathrm{~A})-\mathrm{C}(5)-\mathrm{H}(5 \mathrm{~B})$ & 107.6 & $\mathrm{C}(14)-\mathrm{C}(15)-\mathrm{C}(16)$ & $109.3(6)$ \\
\hline $\mathrm{N}(3)-\mathrm{C}(6)-\mathrm{N}(1)$ & $113.8(6)$ & $\mathrm{C}(14)-\mathrm{C}(15)-\mathrm{Fe}(1)$ & $70.1(4)$ \\
\hline $\mathrm{N}(3)-\mathrm{C}(6)-\mathrm{H}(6 \mathrm{~A})$ & 108.8 & $\mathrm{C}(16)-\mathrm{C}(15)-\mathrm{Fe}(1)$ & $70.4(4)$ \\
\hline $\mathrm{N}(1)-\mathrm{C}(6)-\mathrm{H}(6 \mathrm{~A})$ & 108.8 & $\mathrm{C}(14)-\mathrm{C}(15)-\mathrm{H}(15 \mathrm{~A})$ & 125.4 \\
\hline N(3)-C(6)-H(6B) & 108.8 & $\mathrm{C}(16)-\mathrm{C}(15)-\mathrm{H}(15 \mathrm{~A})$ & 125.4 \\
\hline $\mathrm{N}(1)-\mathrm{C}(6)-\mathrm{H}(6 \mathrm{~B})$ & 108.8 & $\mathrm{Fe}(1)-\mathrm{C}(15)-\mathrm{H}(15 \mathrm{~A})$ & 125.4 \\
\hline $\mathrm{H}(6 \mathrm{~A})-\mathrm{C}(6)-\mathrm{H}(6 \mathrm{~B})$ & 107.7 & $\mathrm{C}(15)-\mathrm{C}(16)-\mathrm{C}(17)$ & $107.3(6)$ \\
\hline $\mathrm{O}(1)-\mathrm{C}(7)-\mathrm{C}(8)$ & $109.6(5)$ & $\mathrm{C}(15)-\mathrm{C}(16)-\mathrm{Fe}(1)$ & $69.2(4)$ \\
\hline $\mathrm{O}(1)-\mathrm{C}(7)-\mathrm{C}(1)$ & $106.7(5)$ & $\mathrm{C}(17)-\mathrm{C}(16)-\mathrm{Fe}(1)$ & $68.9(3)$ \\
\hline$C(8)-C(7)-C(1)$ & $110.9(5)$ & $\mathrm{C}(15)-\mathrm{C}(16)-\mathrm{H}(16 \mathrm{~A})$ & 126.4 \\
\hline $\mathrm{O}(1)-\mathrm{C}(7)-\mathrm{H}(7 \mathrm{~A})$ & 109.9 & $\mathrm{C}(17)-\mathrm{C}(16)-\mathrm{H}(16 \mathrm{~A})$ & 126.4 \\
\hline $\mathrm{C}(8)-\mathrm{C}(7)-\mathrm{H}(7 \mathrm{~A})$ & 109.9 & $\mathrm{Fe}(1)-\mathrm{C}(16)-\mathrm{H}(16 \mathrm{~A})$ & 126.4 \\
\hline $\mathrm{C}(1)-\mathrm{C}(7)-\mathrm{H}(7 \mathrm{~A})$ & 109.9 & $\mathrm{C}(13)-\mathrm{C}(17)-\mathrm{C}(16)$ & 107.2(6) \\
\hline $\mathrm{C}(12)-\mathrm{C}(8)-\mathrm{C}(9)$ & $109.0(6)$ & $\mathrm{C}(13)-\mathrm{C}(17)-\mathrm{Fe}(1)$ & $69.9(3)$ \\
\hline $\mathrm{C}(12)-\mathrm{C}(8)-\mathrm{C}(7)$ & $126.6(6)$ & $\mathrm{C}(16)-\mathrm{C}(17)-\mathrm{Fe}(1)$ & $69.4(4)$ \\
\hline$C(9)-C(8)-C(7)$ & 124.3(6) & $\mathrm{C}(13)-\mathrm{C}(17)-\mathrm{H}(17 \mathrm{~A})$ & 126.4 \\
\hline $\mathrm{C}(12)-\mathrm{C}(8)-\mathrm{Fe}(1)$ & $69.5(4)$ & $\mathrm{C}(16)-\mathrm{C}(17)-\mathrm{H}(17 \mathrm{~A})$ & 126.4 \\
\hline $\mathrm{C}(9)-\mathrm{C}(8)-\mathrm{Fe}(1)$ & 69.9(4) & $\mathrm{Fe}(1)-\mathrm{C}(17)-\mathrm{H}(17 \mathrm{~A})$ & 126.4 \\
\hline
\end{tabular}


Table 8-S. Hydrogen bonds for PTA-CH(ferrocenyl)OH [ $\AA^{\circ}$ and $\left.{ }^{\circ}\right]$.

\begin{tabular}{lcccc}
\hline $\mathrm{D}-\mathrm{H} \ldots \mathrm{A}$ & $\mathrm{d}(\mathrm{D}-\mathrm{H})$ & $\mathrm{d}(\mathrm{H} \ldots \mathrm{A})$ & $\mathrm{d}(\mathrm{D} \ldots \mathrm{A})$ & $<(\mathrm{DHA})$ \\
\hline $\mathrm{O}(1)-\mathrm{H}(1 \mathrm{~A}) \ldots \mathrm{N}(1)$ & $1.03(7)$ & $1.93(7)$ & $2.634(7)$ & $123(6)$ \\
\hline
\end{tabular}

Symmetry transformations used to generate equivalent atoms:

Table 9-S. Bond lengths $[\AA]$ and angles [ $\left.{ }^{\circ}\right]$ for $\left[\left(\eta^{6}-\mathrm{C}_{6} \mathrm{H}_{5} \mathrm{CH}_{3}\right) \mathrm{Ru}\left(\mathrm{PTA}-\mathrm{C}\left(\mathrm{C}_{6} \mathrm{H}_{5}\right)_{2} \mathrm{OH}\right) \mathrm{Cl}_{2}\right]$.

\begin{tabular}{|c|c|c|c|}
\hline $\mathrm{Ru}(1)-\mathrm{C}(22)$ & $2.152(5)$ & $\mathrm{C}(11)-\mathrm{H}(11 \mathrm{~A})$ & 0.9500 \\
\hline $\operatorname{Ru}(1)-C(21)$ & $2.189(4)$ & $\mathrm{C}(12)-\mathrm{C}(13)$ & $1.384(6)$ \\
\hline $\mathrm{Ru}(1)-\mathrm{C}(23)$ & $2.190(5)$ & $\mathrm{C}(12)-\mathrm{H}(12 \mathrm{~A})$ & 0.9500 \\
\hline $\mathrm{Ru}(1)-\mathrm{C}(24)$ & $2.197(4)$ & $\mathrm{C}(13)-\mathrm{H}(13 \mathrm{~A})$ & 0.9500 \\
\hline $\mathrm{Ru}(1)-\mathrm{C}(25)$ & $2.239(4)$ & $C(14)-C(19)$ & $1.391(6)$ \\
\hline $\operatorname{Ru}(1)-C(20)$ & $2.244(4)$ & $C(14)-C(15)$ & $1.394(6)$ \\
\hline $\mathrm{Ru}(1)-\mathrm{P}(1)$ & $2.3294(11)$ & $C(15)-C(16)$ & $1.389(6)$ \\
\hline $\mathrm{Ru}(1)-\mathrm{Cl}(2)$ & $2.4077(12)$ & $\mathrm{C}(15)-\mathrm{H}(15 \mathrm{~A})$ & 0.9500 \\
\hline $\mathrm{Ru}(1)-\mathrm{Cl}(1)$ & $2.4119(12)$ & $C(16)-C(17)$ & $1.379(6)$ \\
\hline$P(1)-C(2)$ & $1.848(4)$ & $\mathrm{C}(16)-\mathrm{H}(16 \mathrm{~A})$ & 0.9500 \\
\hline$P(1)-C(3)$ & $1.852(4)$ & $\mathrm{C}(17)-\mathrm{C}(18)$ & $1.379(7)$ \\
\hline $\mathrm{P}(1)-\mathrm{C}(1)$ & $1.885(4)$ & $\mathrm{C}(17)-\mathrm{H}(17 \mathrm{~A})$ & 0.9500 \\
\hline $\mathrm{O}(1)-\mathrm{C}(7)$ & $1.416(5)$ & $\mathrm{C}(18)-\mathrm{C}(19)$ & $1.389(6)$ \\
\hline $\mathrm{O}(1)-\mathrm{H}(1 \mathrm{~A})$ & $0.81(5)$ & $\mathrm{C}(18)-\mathrm{H}(18 \mathrm{~A})$ & 0.9500 \\
\hline $\mathrm{N}(1)-\mathrm{C}(6)$ & $1.462(5)$ & $\mathrm{C}(19)-\mathrm{H}(19 \mathrm{~A})$ & 0.9500 \\
\hline $\mathrm{N}(1)-\mathrm{C}(4)$ & $1.464(5)$ & $C(20)-C(25)$ & $1.395(7)$ \\
\hline $\mathrm{N}(1)-\mathrm{C}(1)$ & $1.484(5)$ & $C(20)-C(21)$ & $1.423(6)$ \\
\hline $\mathrm{N}(2)-\mathrm{C}(4)$ & $1.463(5)$ & $C(20)-C(26)$ & $1.503(6)$ \\
\hline $\mathrm{N}(2)-\mathrm{C}(5)$ & $1.465(5)$ & $\mathrm{C}(21)-\mathrm{C}(22)$ & $1.410(6)$ \\
\hline $\mathrm{N}(2)-\mathrm{C}(2)$ & $1.481(5)$ & $\mathrm{C}(21)-\mathrm{H}(21 \mathrm{~A})$ & 1.0000 \\
\hline $\mathrm{N}(3)-\mathrm{C}(5)$ & $1.464(5)$ & $\mathrm{C}(22)-\mathrm{C}(23)$ & $1.401(7)$ \\
\hline $\mathrm{N}(3)-\mathrm{C}(6)$ & $1.466(5)$ & $\mathrm{C}(22)-\mathrm{H}(22 \mathrm{~A})$ & 1.0000 \\
\hline $\mathrm{N}(3)-\mathrm{C}(3)$ & $1.472(5)$ & $\mathrm{C}(23)-\mathrm{C}(24)$ & $1.398(6)$ \\
\hline $\mathrm{C}(1)-\mathrm{C}(7)$ & $1.563(6)$ & $\mathrm{C}(23)-\mathrm{H}(23 \mathrm{~A})$ & 1.0000 \\
\hline $\mathrm{C}(1)-\mathrm{H}(1 \mathrm{~B})$ & 1.0000 & $C(24)-C(25)$ & $1.413(6)$ \\
\hline $\mathrm{C}(2)-\mathrm{H}(2 \mathrm{~A})$ & 0.9900 & $\mathrm{C}(24)-\mathrm{H}(24 \mathrm{~A})$ & 1.0000 \\
\hline $\mathrm{C}(2)-\mathrm{H}(2 \mathrm{~B})$ & 0.9900 & $\mathrm{C}(25)-\mathrm{H}(25 \mathrm{~A})$ & 1.0000 \\
\hline $\mathrm{C}(3)-\mathrm{H}(3 \mathrm{~A})$ & 0.9900 & $\mathrm{C}(26)-\mathrm{H}(26 \mathrm{~A})$ & 0.9800 \\
\hline $\mathrm{C}(3)-\mathrm{H}(3 \mathrm{~B})$ & 0.9900 & $\mathrm{C}(26)-\mathrm{H}(26 \mathrm{~B})$ & 0.9800 \\
\hline $\mathrm{C}(4)-\mathrm{H}(4 \mathrm{~A})$ & 0.9900 & $\mathrm{C}(26)-\mathrm{H}(26 \mathrm{C})$ & 0.9800 \\
\hline $\mathrm{C}(4)-\mathrm{H}(4 \mathrm{~B})$ & 0.9900 & & \\
\hline $\mathrm{C}(5)-\mathrm{H}(5 \mathrm{~A})$ & 0.9900 & $\mathrm{C}(22)-\mathrm{Ru}(1)-\mathrm{C}(21)$ & $37.89(16)$ \\
\hline $\mathrm{C}(5)-\mathrm{H}(5 \mathrm{~B})$ & 0.9900 & $C(22)-R u(1)-C(23)$ & $37.64(17)$ \\
\hline $\mathrm{C}(6)-\mathrm{H}(6 \mathrm{~A})$ & 0.9900 & $\mathrm{C}(21)-\mathrm{Ru}(1)-\mathrm{C}(23)$ & $68.14(18)$ \\
\hline $\mathrm{C}(6)-\mathrm{H}(6 \mathrm{~B})$ & 0.9900 & $\mathrm{C}(22)-\mathrm{Ru}(1)-\mathrm{C}(24)$ & $67.04(18)$ \\
\hline$C(7)-C(8)$ & $1.531(6)$ & $\mathrm{C}(21)-\mathrm{Ru}(1)-\mathrm{C}(24)$ & $79.33(18)$ \\
\hline$C(7)-C(14)$ & $1.543(6)$ & $\mathrm{C}(23)-\mathrm{Ru}(1)-\mathrm{C}(24)$ & $37.16(17)$ \\
\hline$C(8)-C(13)$ & $1.386(6)$ & $\mathrm{C}(22)-\mathrm{Ru}(1)-\mathrm{C}(25)$ & $79.12(16)$ \\
\hline $\mathrm{C}(8)-\mathrm{C}(9)$ & $1.387(6)$ & $\mathrm{C}(21)-\mathrm{Ru}(1)-\mathrm{C}(25)$ & $66.60(17)$ \\
\hline $\mathrm{C}(9)-\mathrm{C}(10)$ & $1.383(6)$ & $\mathrm{C}(23)-\mathrm{Ru}(1)-\mathrm{C}(25)$ & $67.21(17)$ \\
\hline $\mathrm{C}(9)-\mathrm{H}(9 \mathrm{~A})$ & 0.9500 & $C(24)-R u(1)-C(25)$ & $37.12(17)$ \\
\hline$C(10)-C(11)$ & $1.380(6)$ & $\mathrm{C}(22)-\mathrm{Ru}(1)-\mathrm{C}(20)$ & $67.60(17)$ \\
\hline $\mathrm{C}(10)-\mathrm{H}(10 \mathrm{~A})$ & 0.9500 & $\mathrm{C}(21)-\mathrm{Ru}(1)-\mathrm{C}(20)$ & $37.43(16)$ \\
\hline $\mathrm{C}(11)-\mathrm{C}(12)$ & $1.382(6)$ & $\mathrm{C}(23)-\mathrm{Ru}(1)-\mathrm{C}(20)$ & $79.74(16)$ \\
\hline
\end{tabular}




\begin{tabular}{|c|c|}
\hline $\mathrm{C}(24)-\mathrm{Ru}(1)-\mathrm{C}(20)$ & $66.44(18)$ \\
\hline $\mathrm{C}(25)-\mathrm{Ru}(1)-\mathrm{C}(20)$ & $36.27(17)$ \\
\hline $\mathrm{C}(22)-\mathrm{Ru}(1)-\mathrm{P}(1)$ & $92.00(12)$ \\
\hline $\mathrm{C}(21)-\mathrm{Ru}(1)-\mathrm{P}(1)$ & $109.74(12)$ \\
\hline $\mathrm{C}(23)-\mathrm{Ru}(1)-\mathrm{P}(1)$ & $102.08(12)$ \\
\hline $\mathrm{C}(24)-\mathrm{Ru}(1)-\mathrm{P}(1)$ & $133.45(13)$ \\
\hline $\mathrm{C}(25)-\mathrm{Ru}(1)-\mathrm{P}(1)$ & $169.28(13)$ \\
\hline $\mathrm{C}(20)-\mathrm{Ru}(1)-\mathrm{P}(1)$ & $144.47(14)$ \\
\hline $\mathrm{C}(22)-\mathrm{Ru}(1)-\mathrm{Cl}(2)$ & $132.15(14)$ \\
\hline $\mathrm{C}(21)-\mathrm{Ru}(1)-\mathrm{Cl}(2)$ & $99.22(13)$ \\
\hline $\mathrm{C}(23)-\mathrm{Ru}(1)-\mathrm{Cl}(2)$ & $167.03(13)$ \\
\hline $\mathrm{C}(24)-\mathrm{Ru}(1)-\mathrm{Cl}(2)$ & $140.51(13)$ \\
\hline $\mathrm{C}(25)-\mathrm{Ru}(1)-\mathrm{Cl}(2)$ & $105.77(13)$ \\
\hline $\mathrm{C}(20)-\mathrm{Ru}(1)-\mathrm{Cl}(2)$ & $88.35(12)$ \\
\hline $\mathrm{P}(1)-\mathrm{Ru}(1)-\mathrm{Cl}(2)$ & $84.63(4)$ \\
\hline $\mathrm{C}(22)-\mathrm{Ru}(1)-\mathrm{Cl}(1)$ & $138.38(14)$ \\
\hline $\mathrm{C}(21)-\mathrm{Ru}(1)-\mathrm{Cl}(1)$ & $164.77(12)$ \\
\hline $\mathrm{C}(23)-\mathrm{Ru}(1)-\mathrm{Cl}(1)$ & $102.87(14)$ \\
\hline $\mathrm{C}(24)-\mathrm{Ru}(1)-\mathrm{Cl}(1)$ & $86.24(13)$ \\
\hline $\mathrm{C}(25)-\mathrm{Ru}(1)-\mathrm{Cl}(1)$ & $98.83(13)$ \\
\hline $\mathrm{C}(20)-\mathrm{Ru}(1)-\mathrm{Cl}(1)$ & $130.95(14)$ \\
\hline $\mathrm{P}(1)-\mathrm{Ru}(1)-\mathrm{Cl}(1)$ & $83.76(4)$ \\
\hline $\mathrm{Cl}(2)-\mathrm{Ru}(1)-\mathrm{Cl}(1)$ & $88.79(4)$ \\
\hline $\mathrm{C}(2)-\mathrm{P}(1)-\mathrm{C}(3)$ & $98.3(2)$ \\
\hline $\mathrm{C}(2)-\mathrm{P}(1)-\mathrm{C}(1)$ & $97.51(18)$ \\
\hline $\mathrm{C}(3)-\mathrm{P}(1)-\mathrm{C}(1)$ & $100.59(19)$ \\
\hline $\mathrm{C}(2)-\mathrm{P}(1)-\mathrm{Ru}(1)$ & $112.36(13)$ \\
\hline $\mathrm{C}(3)-\mathrm{P}(1)-\mathrm{Ru}(1)$ & $117.66(13)$ \\
\hline $\mathrm{C}(1)-\mathrm{P}(1)-\mathrm{Ru}(1)$ & $125.54(13)$ \\
\hline $\mathrm{C}(7)-\mathrm{O}(1)-\mathrm{H}(1 \mathrm{~A})$ & $112(4)$ \\
\hline $\mathrm{C}(6)-\mathrm{N}(1)-\mathrm{C}(4)$ & $108.8(3)$ \\
\hline $\mathrm{C}(6)-\mathrm{N}(1)-\mathrm{C}(1)$ & 114.1(3) \\
\hline $\mathrm{C}(4)-\mathrm{N}(1)-\mathrm{C}(1)$ & $111.5(3)$ \\
\hline $\mathrm{C}(4)-\mathrm{N}(2)-\mathrm{C}(5)$ & $108.0(3)$ \\
\hline $\mathrm{C}(4)-\mathrm{N}(2)-\mathrm{C}(2)$ & $109.7(3)$ \\
\hline $\mathrm{C}(5)-\mathrm{N}(2)-\mathrm{C}(2)$ & $110.5(3)$ \\
\hline $\mathrm{C}(5)-\mathrm{N}(3)-\mathrm{C}(6)$ & $109.0(3)$ \\
\hline $\mathrm{C}(5)-\mathrm{N}(3)-\mathrm{C}(3)$ & $111.9(3)$ \\
\hline $\mathrm{C}(6)-\mathrm{N}(3)-\mathrm{C}(3)$ & $109.9(3)$ \\
\hline $\mathrm{N}(1)-\mathrm{C}(1)-\mathrm{C}(7)$ & $113.1(3)$ \\
\hline $\mathrm{N}(1)-\mathrm{C}(1)-\mathrm{P}(1)$ & $107.6(3)$ \\
\hline $\mathrm{C}(7)-\mathrm{C}(1)-\mathrm{P}(1)$ & $115.9(3)$ \\
\hline $\mathrm{N}(1)-\mathrm{C}(1)-\mathrm{H}(1 \mathrm{~B})$ & 106.6 \\
\hline $\mathrm{C}(7)-\mathrm{C}(1)-\mathrm{H}(1 \mathrm{~B})$ & 106.6 \\
\hline $\mathrm{P}(1)-\mathrm{C}(1)-\mathrm{H}(1 \mathrm{~B})$ & 106.6 \\
\hline $\mathrm{N}(2)-\mathrm{C}(2)-\mathrm{P}(1)$ & $112.9(3)$ \\
\hline $\mathrm{N}(2)-\mathrm{C}(2)-\mathrm{H}(2 \mathrm{~A})$ & 109.0 \\
\hline $\mathrm{P}(1)-\mathrm{C}(2)-\mathrm{H}(2 \mathrm{~A})$ & 109.0 \\
\hline $\mathrm{N}(2)-\mathrm{C}(2)-\mathrm{H}(2 \mathrm{~B})$ & 109.0 \\
\hline $\mathrm{P}(1)-\mathrm{C}(2)-\mathrm{H}(2 \mathrm{~B})$ & 109.0 \\
\hline $\mathrm{H}(2 \mathrm{~A})-\mathrm{C}(2)-\mathrm{H}(2 \mathrm{~B})$ & 107.8 \\
\hline $\mathrm{N}(3)-\mathrm{C}(3)-\mathrm{P}(1)$ & $111.4(3)$ \\
\hline $\mathrm{N}(3)-\mathrm{C}(3)-\mathrm{H}(3 \mathrm{~A})$ & 109.4 \\
\hline $\mathrm{P}(1)-\mathrm{C}(3)-\mathrm{H}(3 \mathrm{~A})$ & 109.4 \\
\hline $\mathrm{N}(3)-\mathrm{C}(3)-\mathrm{H}(3 \mathrm{~B})$ & 109.4 \\
\hline $\mathrm{P}(1)-\mathrm{C}(3)-\mathrm{H}(3 \mathrm{~B})$ & 109.4 \\
\hline
\end{tabular}

\begin{tabular}{|c|c|}
\hline $\mathrm{H}(3 \mathrm{~A})-\mathrm{C}(3)-\mathrm{H}(3 \mathrm{~B})$ & 108.0 \\
\hline $\mathrm{N}(2)-\mathrm{C}(4)-\mathrm{N}(1)$ & $114.9(3)$ \\
\hline $\mathrm{N}(2)-\mathrm{C}(4)-\mathrm{H}(4 \mathrm{~A})$ & 108.5 \\
\hline $\mathrm{N}(1)-\mathrm{C}(4)-\mathrm{H}(4 \mathrm{~A})$ & 108.5 \\
\hline $\mathrm{N}(2)-\mathrm{C}(4)-\mathrm{H}(4 \mathrm{~B})$ & 108.5 \\
\hline $\mathrm{N}(1)-\mathrm{C}(4)-\mathrm{H}(4 \mathrm{~B})$ & 108.5 \\
\hline $\mathrm{H}(4 \mathrm{~A})-\mathrm{C}(4)-\mathrm{H}(4 \mathrm{~B})$ & 107.5 \\
\hline $\mathrm{N}(3)-\mathrm{C}(5)-\mathrm{N}(2)$ & $115.2(3)$ \\
\hline $\mathrm{N}(3)-\mathrm{C}(5)-\mathrm{H}(5 \mathrm{~A})$ & 108.5 \\
\hline $\mathrm{N}(2)-\mathrm{C}(5)-\mathrm{H}(5 \mathrm{~A})$ & 108.5 \\
\hline $\mathrm{N}(3)-\mathrm{C}(5)-\mathrm{H}(5 \mathrm{~B})$ & 108.5 \\
\hline $\mathrm{N}(2)-\mathrm{C}(5)-\mathrm{H}(5 \mathrm{~B})$ & 108.5 \\
\hline $\mathrm{H}(5 \mathrm{~A})-\mathrm{C}(5)-\mathrm{H}(5 \mathrm{~B})$ & 107.5 \\
\hline $\mathrm{N}(1)-\mathrm{C}(6)-\mathrm{N}(3)$ & $114.1(3)$ \\
\hline $\mathrm{N}(1)-\mathrm{C}(6)-\mathrm{H}(6 \mathrm{~A})$ & 108.7 \\
\hline $\mathrm{N}(3)-\mathrm{C}(6)-\mathrm{H}(6 \mathrm{~A})$ & 108.7 \\
\hline $\mathrm{N}(1)-\mathrm{C}(6)-\mathrm{H}(6 \mathrm{~B})$ & 108.7 \\
\hline $\mathrm{N}(3)-\mathrm{C}(6)-\mathrm{H}(6 \mathrm{~B})$ & 108.7 \\
\hline $\mathrm{H}(6 \mathrm{~A})-\mathrm{C}(6)-\mathrm{H}(6 \mathrm{~B})$ & 107.6 \\
\hline $\mathrm{O}(1)-\mathrm{C}(7)-\mathrm{C}(8)$ & $105.7(3)$ \\
\hline $\mathrm{O}(1)-\mathrm{C}(7)-\mathrm{C}(14)$ & $110.6(3)$ \\
\hline $\mathrm{C}(8)-\mathrm{C}(7)-\mathrm{C}(14)$ & $106.9(3)$ \\
\hline $\mathrm{O}(1)-\mathrm{C}(7)-\mathrm{C}(1)$ & $110.8(3)$ \\
\hline$C(8)-C(7)-C(1)$ & $110.8(3)$ \\
\hline $\mathrm{C}(14)-\mathrm{C}(7)-\mathrm{C}(1)$ & $111.9(3)$ \\
\hline $\mathrm{C}(13)-\mathrm{C}(8)-\mathrm{C}(9)$ & $118.2(4)$ \\
\hline $\mathrm{C}(13)-\mathrm{C}(8)-\mathrm{C}(7)$ & $121.0(4)$ \\
\hline$C(9)-C(8)-C(7)$ & $120.7(4)$ \\
\hline $\mathrm{C}(10)-\mathrm{C}(9)-\mathrm{C}(8)$ & $120.9(4)$ \\
\hline $\mathrm{C}(10)-\mathrm{C}(9)-\mathrm{H}(9 \mathrm{~A})$ & 119.5 \\
\hline $\mathrm{C}(8)-\mathrm{C}(9)-\mathrm{H}(9 \mathrm{~A})$ & 119.5 \\
\hline $\mathrm{C}(11)-\mathrm{C}(10)-\mathrm{C}(9)$ & $120.1(4)$ \\
\hline $\mathrm{C}(11)-\mathrm{C}(10)-\mathrm{H}(10 \mathrm{~A})$ & 120.0 \\
\hline $\mathrm{C}(9)-\mathrm{C}(10)-\mathrm{H}(10 \mathrm{~A})$ & 120.0 \\
\hline$C(10)-C(11)-C(12)$ & $119.8(4)$ \\
\hline $\mathrm{C}(10)-\mathrm{C}(11)-\mathrm{H}(11 \mathrm{~A})$ & 120.1 \\
\hline $\mathrm{C}(12)-\mathrm{C}(11)-\mathrm{H}(11 \mathrm{~A})$ & 120.1 \\
\hline $\mathrm{C}(11)-\mathrm{C}(12)-\mathrm{C}(13)$ & $119.6(4)$ \\
\hline $\mathrm{C}(11)-\mathrm{C}(12)-\mathrm{H}(12 \mathrm{~A})$ & 120.2 \\
\hline $\mathrm{C}(13)-\mathrm{C}(12)-\mathrm{H}(12 \mathrm{~A})$ & 120.2 \\
\hline$C(12)-C(13)-C(8)$ & $121.3(4)$ \\
\hline $\mathrm{C}(12)-\mathrm{C}(13)-\mathrm{H}(13 \mathrm{~A})$ & 119.3 \\
\hline $\mathrm{C}(8)-\mathrm{C}(13)-\mathrm{H}(13 \mathrm{~A})$ & 119.3 \\
\hline$C(19)-C(14)-C(15)$ & $117.3(4)$ \\
\hline$C(19)-C(14)-C(7)$ & $119.4(4)$ \\
\hline$C(15)-C(14)-C(7)$ & $123.0(4)$ \\
\hline$C(16)-C(15)-C(14)$ & $121.4(4)$ \\
\hline $\mathrm{C}(16)-\mathrm{C}(15)-\mathrm{H}(15 \mathrm{~A})$ & 119.3 \\
\hline $\mathrm{C}(14)-\mathrm{C}(15)-\mathrm{H}(15 \mathrm{~A})$ & 119.3 \\
\hline $\mathrm{C}(17)-\mathrm{C}(16)-\mathrm{C}(15)$ & $120.1(5)$ \\
\hline $\mathrm{C}(17)-\mathrm{C}(16)-\mathrm{H}(16 \mathrm{~A})$ & 119.9 \\
\hline $\mathrm{C}(15)-\mathrm{C}(16)-\mathrm{H}(16 \mathrm{~A})$ & 119.9 \\
\hline $\mathrm{C}(18)-\mathrm{C}(17)-\mathrm{C}(16)$ & $119.6(4)$ \\
\hline $\mathrm{C}(18)-\mathrm{C}(17)-\mathrm{H}(17 \mathrm{~A})$ & 120.2 \\
\hline $\mathrm{C}(16)-\mathrm{C}(17)-\mathrm{H}(17 \mathrm{~A})$ & 120.2 \\
\hline $\mathrm{C}(17)-\mathrm{C}(18)-\mathrm{C}(19)$ & $120.1(4)$ \\
\hline
\end{tabular}




$\begin{array}{llll}\mathrm{C}(17)-\mathrm{C}(18)-\mathrm{H}(18 \mathrm{~A}) & 120.0 & \mathrm{C}(24)-\mathrm{C}(23)-\mathrm{Ru}(1) & 71.7(3) \\ \mathrm{C}(19)-\mathrm{C}(18)-\mathrm{H}(18 \mathrm{~A}) & 120.0 & \mathrm{C}(22)-\mathrm{C}(23)-\mathrm{Ru}(1) & 69.7(3) \\ \mathrm{C}(18)-\mathrm{C}(19)-\mathrm{C}(14) & 121.5(5) & \mathrm{C}(24)-\mathrm{C}(23)-\mathrm{H}(23 \mathrm{~A}) & 120.4 \\ \mathrm{C}(18)-\mathrm{C}(19)-\mathrm{H}(19 \mathrm{~A}) & 119.2 & \mathrm{C}(22)-\mathrm{C}(23)-\mathrm{H}(23 \mathrm{~A}) & 120.4 \\ \mathrm{C}(14)-\mathrm{C}(19)-\mathrm{H}(19 \mathrm{~A}) & 119.2 & \mathrm{Ru}(1)-\mathrm{C}(23)-\mathrm{H}(23 \mathrm{~A}) & 120.4 \\ \mathrm{C}(25)-\mathrm{C}(20)-\mathrm{C}(21) & 119.2(4) & \mathrm{C}(23)-\mathrm{C}(24)-\mathrm{C}(25) & 121.4(5) \\ \mathrm{C}(25)-\mathrm{C}(20)-\mathrm{C}(26) & 121.0(5) & \mathrm{C}(23)-\mathrm{C}(24)-\mathrm{Ru}(1) & 71.1(3) \\ \mathrm{C}(21)-\mathrm{C}(20)-\mathrm{C}(26) & 119.7(5) & \mathrm{C}(25)-\mathrm{C}(24)-\mathrm{Ru}(1) & 73.0(3) \\ \mathrm{C}(25)-\mathrm{C}(20)-\mathrm{Ru}(1) & 71.6(3) & \mathrm{C}(23)-\mathrm{C}(24)-\mathrm{H}(24 \mathrm{~A}) & 118.8 \\ \mathrm{C}(21)-\mathrm{C}(20)-\mathrm{Ru}(1) & 69.2(2) & \mathrm{C}(25)-\mathrm{C}(24)-\mathrm{H}(24 \mathrm{~A}) & 118.8 \\ \mathrm{C}(26)-\mathrm{C}(20)-\mathrm{Ru}(1) & 128.1(3) & \mathrm{Ru}(1)-\mathrm{C}(24)-\mathrm{H}(24 \mathrm{~A}) & 118.8 \\ \mathrm{C}(22)-\mathrm{C}(21)-\mathrm{C}(20) & 119.4(5) & \mathrm{C}(20)-\mathrm{C}(25)-\mathrm{C}(24) & 120.2(4) \\ \mathrm{C}(22)-\mathrm{C}(21)-\mathrm{Ru}(1) & 69.6(3) & \mathrm{C}(20)-\mathrm{C}(25)-\mathrm{Ru}(1) & 72.1(2) \\ \mathrm{C}(20)-\mathrm{C}(21)-\mathrm{Ru}(1) & 73.4(3) & \mathrm{C}(24)-\mathrm{C}(25)-\mathrm{Ru}(1) & 69.9(2) \\ \mathrm{C}(22)-\mathrm{C}(21)-\mathrm{H}(21 \mathrm{~A}) & 119.9 & \mathrm{C}(20)-\mathrm{C}(25)-\mathrm{H}(25 \mathrm{~A}) & 119.2 \\ \mathrm{C}(20)-\mathrm{C}(21)-\mathrm{H}(21 \mathrm{~A}) & 119.9 & \mathrm{C}(24)-\mathrm{C}(25)-\mathrm{H}(25 \mathrm{~A}) & 119.2 \\ \mathrm{Ru}(1)-\mathrm{C}(21)-\mathrm{H}(21 \mathrm{~A}) & 119.9 & \mathrm{Ru}(1)-\mathrm{C}(25)-\mathrm{H}(25 \mathrm{~A}) & 119.2 \\ \mathrm{C}(23)-\mathrm{C}(22)-\mathrm{C}(21) & 121.6(4) & \mathrm{C}(20)-\mathrm{C}(26)-\mathrm{H}(26 \mathrm{~A}) & 109.5 \\ \mathrm{C}(23)-\mathrm{C}(22)-\mathrm{Ru}(1) & 72.7(3) & \mathrm{C}(20)-\mathrm{C}(26)-\mathrm{H}(26 \mathrm{~B}) & 109.5 \\ \mathrm{C}(21)-\mathrm{C}(22)-\mathrm{Ru}(1) & 72.5(3) & \mathrm{H}(26 \mathrm{~A})-\mathrm{C}(26)-\mathrm{H}(26 \mathrm{~B}) & 109.5 \\ \mathrm{C}(23)-\mathrm{C}(22)-\mathrm{H}(22 \mathrm{~A}) & 118.8 & \mathrm{C}(20)-\mathrm{C}(26)-\mathrm{H}(26 \mathrm{C}) & 109.5 \\ \mathrm{C}(21)-\mathrm{C}(22)-\mathrm{H}(22 \mathrm{~A}) & 118.8 & \mathrm{H}(26 \mathrm{~A})-\mathrm{C}(26)-\mathrm{H}(26 \mathrm{C}) & 109.5 \\ \mathrm{Ru}(1)-\mathrm{C}(22)-\mathrm{H}(22 \mathrm{~A}) & 118.8 & \mathrm{H}(26 \mathrm{~B})-\mathrm{C}(26)-\mathrm{H}(26 \mathrm{C}) & 109.5 \\ \mathrm{C}(24)-\mathrm{C}(23)-\mathrm{C}(22) & 118.2(4) & & \end{array}$

Table 10-S. Hydrogen bonds for $\left.\left[\left(\eta^{6}-\mathrm{C}_{6} \mathrm{H}_{5} \mathrm{CH}_{3}\right) \mathrm{Ru}\left(\mathrm{PTA}-\mathrm{C}\left(\mathrm{C}_{6} \mathrm{H}_{5}\right)_{2} \mathrm{OH}\right) \mathrm{Cl}_{2}\right)\right]$.

\begin{tabular}{lcccc}
\hline $\mathrm{D}-\mathrm{H} \ldots \mathrm{A}$ & $\mathrm{d}(\mathrm{D}-\mathrm{H})$ & $\mathrm{d}(\mathrm{H} \ldots \mathrm{A})$ & $\mathrm{d}(\mathrm{D} \ldots \mathrm{A})$ & $<(\mathrm{DHA})$ \\
\hline $\mathrm{O}(1)-\mathrm{H}(1 \mathrm{~A}) \ldots \mathrm{Cl}(2)$ & $0.81(5)$ & $2.31(5)$ & $3.100(3)$ & $167(5)$ \\
\hline
\end{tabular}

Table 11-S. Bond lengths $[\AA]$ and angles $\left[^{\circ}\right]$ for $\left(\eta^{6}-\mathrm{C}_{6} \mathrm{H}_{6}\right) \mathrm{RuCl}_{2}\left(\mathrm{PTA}-\mathrm{C}\left(\mathrm{C}_{6} \mathrm{H}_{4} \mathrm{OCH}_{3}\right)_{2} \mathrm{OH}\right)$.

\begin{tabular}{llll}
\hline $\mathrm{Ru}(1)-\mathrm{C}(26)$ & $2.172(4)$ & $\mathrm{N}(2)-\mathrm{C}(2)$ & $1.470(5)$ \\
$\mathrm{Ru}(1)-\mathrm{C}(24)$ & $2.181(4)$ & $\mathrm{N}(2)-\mathrm{C}(5)$ & $1.475(5)$ \\
$\mathrm{Ru}(1)-\mathrm{C}(27)$ & $2.192(4)$ & $\mathrm{N}(3)-\mathrm{C}(6)$ & $1.474(5)$ \\
$\mathrm{Ru}(1)-\mathrm{C}(25)$ & $2.195(4)$ & $\mathrm{N}(3)-\mathrm{C}(3)$ & $1.474(5)$ \\
$\mathrm{Ru}(1)-\mathrm{C}(22)$ & $2.235(4)$ & $\mathrm{N}(3)-\mathrm{C}(5)$ & $1.475(5)$ \\
$\mathrm{Ru}(1)-\mathrm{C}(23)$ & $2.249(4)$ & $\mathrm{O}(1)-\mathrm{C}(7)$ & $1.428(5)$ \\
$\mathrm{Ru}(1)-\mathrm{P}(1)$ & $2.3255(11)$ & $\mathrm{O}(1)-\mathrm{H}(1)$ & $0.75(5)$ \\
$\mathrm{Ru}(1)-\mathrm{Cl}(2)$ & $2.4184(11)$ & $\mathrm{O}(2)-\mathrm{C}(11)$ & $1.376(6)$ \\
$\mathrm{Ru}(1)-\mathrm{Cl}(1)$ & $2.4343(10)$ & $\mathrm{O}(2)-\mathrm{C}(14)$ & $1.428(7)$ \\
$\mathrm{P}(1)-\mathrm{C}(2)$ & $1.843(4)$ & $\mathrm{O}(3)-\mathrm{C}(18)$ & $1.371(5)$ \\
$\mathrm{P}(1)-\mathrm{C}(3)$ & $1.850(4)$ & $\mathrm{O}(3)-\mathrm{C}(21)$ & $1.421(6)$ \\
$\mathrm{P}(1)-\mathrm{C}(1)$ & $1.888(4)$ & $\mathrm{C}(1)-\mathrm{C}(7)$ & $1.563(6)$ \\
$\mathrm{N}(1)-\mathrm{C}(6)$ & $1.463(5)$ & $\mathrm{C}(1)-\mathrm{H}(1 \mathrm{~B})$ & 0.0000 \\
$\mathrm{~N}(1)-\mathrm{C}(4)$ & $1.474(5)$ & $\mathrm{C}(2)-\mathrm{H}(2 \mathrm{~A})$ & 0.99000 \\
$\mathrm{~N}(1)-\mathrm{C}(1)$ & $1.477(5)$ & $\mathrm{C}(2)-\mathrm{H}(2 \mathrm{~B})$ & 0.9900 \\
$\mathrm{~N}(2)-\mathrm{C}(4)$ & $1.469(5)$ & $\mathrm{C}(3)-\mathrm{H}(3 \mathrm{~A})$ &
\end{tabular}




\begin{tabular}{|c|c|c|c|}
\hline$C(3)-H(3 B)$ & 0.9900 & $\mathrm{P}(2)-\mathrm{C}(30)$ & $1.851(4)$ \\
\hline $\mathrm{C}(4)-\mathrm{H}(4 \mathrm{~A})$ & 0.9900 & $\mathrm{P}(2)-\mathrm{C}(29)$ & $1.852(4)$ \\
\hline $\mathrm{C}(4)-\mathrm{H}(4 \mathrm{~B})$ & 0.9900 & $\mathrm{P}(2)-\mathrm{C}(28)$ & $1.887(4)$ \\
\hline $\mathrm{C}(5)-\mathrm{H}(5 \mathrm{~A})$ & 0.9900 & $\mathrm{~N}(4)-\mathrm{C}(33)$ & $1.466(5)$ \\
\hline $\mathrm{C}(5)-\mathrm{H}(5 \mathrm{~B})$ & 0.9900 & $\mathrm{~N}(4)-\mathrm{C}(28)$ & $1.471(5)$ \\
\hline $\mathrm{C}(6)-\mathrm{H}(6 \mathrm{~A})$ & 0.9900 & $\mathrm{~N}(4)-\mathrm{C}(31)$ & $1.471(5)$ \\
\hline $\mathrm{C}(6)-\mathrm{H}(6 \mathrm{~B})$ & 0.9900 & $\mathrm{~N}(5)-\mathrm{C}(29)$ & $1.473(5)$ \\
\hline$C(7)-C(15)$ & $1.544(6)$ & $\mathrm{N}(5)-\mathrm{C}(32)$ & $1.474(6)$ \\
\hline $\mathrm{C}(7)-\mathrm{C}(8)$ & $1.550(6)$ & $\mathrm{N}(5)-\mathrm{C}(31)$ & $1.479(5)$ \\
\hline $\mathrm{C}(8)-\mathrm{C}(13)$ & $1.381(6)$ & $\mathrm{N}(6)-\mathrm{C}(30)$ & $1.462(5)$ \\
\hline $\mathrm{C}(8)-\mathrm{C}(9)$ & $1.387(6)$ & $\mathrm{N}(6)-\mathrm{C}(32)$ & $1.467(5)$ \\
\hline$C(9)-C(10)$ & $1.395(6)$ & $\mathrm{N}(6)-\mathrm{C}(33)$ & $1.467(5)$ \\
\hline $\mathrm{C}(9)-\mathrm{H}(9 \mathrm{~A})$ & 0.9500 & $\mathrm{O}(4)-\mathrm{C}(34)$ & $1.434(5)$ \\
\hline $\mathrm{C}(10)-\mathrm{C}(11)$ & $1.379(7)$ & $\mathrm{O}(4)-\mathrm{H}(4)$ & $0.74(6)$ \\
\hline $\mathrm{C}(10)-\mathrm{H}(10 \mathrm{~A})$ & 0.9500 & $\mathrm{O}(5)-\mathrm{C}(38)$ & $1.373(5)$ \\
\hline$C(11)-C(12)$ & $1.381(8)$ & $\mathrm{O}(5)-\mathrm{C}(41)$ & $1.439(5)$ \\
\hline$C(12)-C(13)$ & $1.393(7)$ & $\mathrm{O}(6)-\mathrm{C}(45)$ & $1.358(5)$ \\
\hline $\mathrm{C}(12)-\mathrm{H}(12 \mathrm{~A})$ & 0.9500 & $\mathrm{O}(6)-\mathrm{C}(48)$ & $1.422(6)$ \\
\hline $\mathrm{C}(13)-\mathrm{H}(13 \mathrm{~A})$ & 0.9500 & $\mathrm{C}(28)-\mathrm{C}(34)$ & $1.567(6)$ \\
\hline $\mathrm{C}(14)-\mathrm{H}(14 \mathrm{~A})$ & 0.9800 & $\mathrm{C}(28)-\mathrm{H}(28 \mathrm{~A})$ & 1.0000 \\
\hline $\mathrm{C}(14)-\mathrm{H}(14 \mathrm{~B})$ & 0.9800 & $\mathrm{C}(29)-\mathrm{H}(29 \mathrm{~A})$ & 0.9900 \\
\hline $\mathrm{C}(14)-\mathrm{H}(14 \mathrm{C})$ & 0.9800 & $\mathrm{C}(29)-\mathrm{H}(29 \mathrm{~B})$ & 0.9900 \\
\hline$C(15)-C(20)$ & $1.390(6)$ & $\mathrm{C}(30)-\mathrm{H}(30 \mathrm{~A})$ & 0.9900 \\
\hline$C(15)-C(16)$ & $1.397(6)$ & $\mathrm{C}(30)-\mathrm{H}(30 \mathrm{~B})$ & 0.9900 \\
\hline$C(16)-C(17)$ & $1.383(6)$ & $\mathrm{C}(31)-\mathrm{H}(31 \mathrm{~A})$ & 0.9900 \\
\hline $\mathrm{C}(16)-\mathrm{H}(16 \mathrm{~A})$ & 0.9500 & $\mathrm{C}(31)-\mathrm{H}(31 \mathrm{~B})$ & 0.9900 \\
\hline$C(17)-C(18)$ & $1.384(6)$ & $\mathrm{C}(32)-\mathrm{H}(32 \mathrm{~A})$ & 0.9900 \\
\hline $\mathrm{C}(17)-\mathrm{H}(17 \mathrm{~A})$ & 0.9500 & $\mathrm{C}(32)-\mathrm{H}(32 \mathrm{~B})$ & 0.9900 \\
\hline$C(18)-C(19)$ & $1.390(6)$ & $\mathrm{C}(33)-\mathrm{H}(33 \mathrm{~A})$ & 0.9900 \\
\hline$C(19)-C(20)$ & $1.394(6)$ & $\mathrm{C}(33)-\mathrm{H}(33 \mathrm{~B})$ & 0.9900 \\
\hline $\mathrm{C}(19)-\mathrm{H}(19 \mathrm{~A})$ & 0.9500 & $\mathrm{C}(34)-\mathrm{C}(42)$ & $1.530(6)$ \\
\hline $\mathrm{C}(20)-\mathrm{H}(20 \mathrm{~A})$ & 0.9500 & $\mathrm{C}(34)-\mathrm{C}(35)$ & $1.534(6)$ \\
\hline $\mathrm{C}(21)-\mathrm{H}(21 \mathrm{~A})$ & 0.9800 & $C(35)-C(36)$ & $1.385(6)$ \\
\hline $\mathrm{C}(21)-\mathrm{H}(21 \mathrm{~B})$ & 0.9800 & $C(35)-C(40)$ & $1.397(6)$ \\
\hline $\mathrm{C}(21)-\mathrm{H}(21 \mathrm{C})$ & 0.9800 & $\mathrm{C}(36)-\mathrm{C}(37)$ & $1.388(6)$ \\
\hline$C(22)-C(23)$ & $1.394(6)$ & $\mathrm{C}(36)-\mathrm{H}(36 \mathrm{~A})$ & 0.9500 \\
\hline$C(22)-C(27)$ & $1.424(6)$ & C(37)-C(38) & $1.381(6)$ \\
\hline $\mathrm{C}(22)-\mathrm{H}(22 \mathrm{~A})$ & 1.0000 & $\mathrm{C}(37)-\mathrm{H}(37 \mathrm{~A})$ & 0.9500 \\
\hline $\mathrm{C}(23)-\mathrm{C}(24)$ & $1.429(6)$ & $\mathrm{C}(38)-\mathrm{C}(39)$ & $1.388(6)$ \\
\hline $\mathrm{C}(23)-\mathrm{H}(23 \mathrm{~A})$ & 1.0000 & $\mathrm{C}(39)-\mathrm{C}(40)$ & $1.388(6)$ \\
\hline$C(24)-C(25)$ & $1.407(6)$ & $\mathrm{C}(39)-\mathrm{H}(39 \mathrm{~A})$ & 0.9500 \\
\hline $\mathrm{C}(24)-\mathrm{H}(24 \mathrm{~A})$ & 1.0000 & $\mathrm{C}(40)-\mathrm{H}(40 \mathrm{~A})$ & 0.9500 \\
\hline$C(25)-C(26)$ & $1.422(6)$ & $\mathrm{C}(41)-\mathrm{H}(41 \mathrm{~A})$ & 0.9800 \\
\hline $\mathrm{C}(25)-\mathrm{H}(25 \mathrm{~A})$ & 1.0000 & $\mathrm{C}(41)-\mathrm{H}(41 \mathrm{~B})$ & 0.9800 \\
\hline$C(26)-C(27)$ & $1.399(6)$ & $\mathrm{C}(41)-\mathrm{H}(41 \mathrm{C})$ & 0.9800 \\
\hline $\mathrm{C}(26)-\mathrm{H}(26 \mathrm{~A})$ & 1.0000 & $\mathrm{C}(42)-\mathrm{C}(47)$ & 1.393(6) \\
\hline $\mathrm{C}(27)-\mathrm{H}(27 \mathrm{~A})$ & 1.0000 & $C(42)-C(43)$ & $1.402(6)$ \\
\hline $\mathrm{Ru}(2)-\mathrm{C}(51)$ & $2.177(4)$ & $\mathrm{C}(43)-\mathrm{C}(44)$ & $1.381(6)$ \\
\hline $\mathrm{Ru}(2)-\mathrm{C}(49)$ & $2.185(4)$ & $\mathrm{C}(43)-\mathrm{H}(43 \mathrm{~A})$ & 0.9500 \\
\hline $\mathrm{Ru}(2)-\mathrm{C}(54)$ & $2.197(4)$ & $\mathrm{C}(44)-\mathrm{C}(45)$ & $1.393(6)$ \\
\hline $\mathrm{Ru}(2)-\mathrm{C}(50)$ & $2.211(4)$ & $\mathrm{C}(44)-\mathrm{H}(44 \mathrm{~A})$ & 0.9500 \\
\hline $\mathrm{Ru}(2)-\mathrm{C}(53)$ & $2.225(5)$ & $C(45)-C(46)$ & $1.395(6)$ \\
\hline $\mathrm{Ru}(2)-\mathrm{C}(52)$ & $2.238(4)$ & $\mathrm{C}(46)-\mathrm{C}(47)$ & $1.384(6)$ \\
\hline $\mathrm{Ru}(2)-\mathrm{P}(2)$ & $2.3448(11)$ & $\mathrm{C}(46)-\mathrm{H}(46 \mathrm{~A})$ & 0.9500 \\
\hline $\mathrm{Ru}(2)-\mathrm{Cl}(4)$ & $2.4150(11)$ & $\mathrm{C}(47)-\mathrm{H}(47 \mathrm{~A})$ & 0.9500 \\
\hline $\mathrm{Ru}(2)-\mathrm{Cl}(3)$ & $2.4172(11)$ & $\mathrm{C}(48)-\mathrm{H}(48 \mathrm{~A})$ & 0.9800 \\
\hline
\end{tabular}




\begin{tabular}{|c|c|}
\hline $\mathrm{C}(48)-\mathrm{H}(48 \mathrm{~B})$ & 0.9800 \\
\hline $\mathrm{C}(48)-\mathrm{H}(48 \mathrm{C})$ & 0.9800 \\
\hline$C(49)-C(50)$ & $1.409(6)$ \\
\hline$C(49)-C(54)$ & $1.411(7)$ \\
\hline $\mathrm{C}(49)-\mathrm{H}(49 \mathrm{~A})$ & 1.0000 \\
\hline$C(50)-C(51)$ & $1.390(6)$ \\
\hline $\mathrm{C}(50)-\mathrm{H}(50 \mathrm{~A})$ & 1.0000 \\
\hline $\mathrm{C}(51)-\mathrm{C}(52)$ & $1.413(6)$ \\
\hline $\mathrm{C}(51)-\mathrm{H}(51 \mathrm{~A})$ & 1.0000 \\
\hline $\mathrm{C}(52)-\mathrm{C}(53)$ & $1.397(6)$ \\
\hline $\mathrm{C}(52)-\mathrm{H}(52 \mathrm{~A})$ & 1.0000 \\
\hline$C(53)-C(54)$ & $1.424(7)$ \\
\hline $\mathrm{C}(53)-\mathrm{H}(53 \mathrm{~A})$ & 1.0000 \\
\hline $\mathrm{C}(54)-\mathrm{H}(54 \mathrm{~A})$ & 1.0000 \\
\hline $\mathrm{Cl}(3 \mathrm{~S})-\mathrm{C}(1 \mathrm{~S})$ & $1.752(5)$ \\
\hline $\mathrm{Cl}(2 \mathrm{~S})-\mathrm{C}(1 \mathrm{~S})$ & $1.766(5)$ \\
\hline $\mathrm{Cl}(1 \mathrm{~S})-\mathrm{C}(1 \mathrm{~S})$ & $1.767(6)$ \\
\hline $\mathrm{C}(1 \mathrm{~S})-\mathrm{H}(1 \mathrm{SA})$ & 1.0000 \\
\hline $\mathrm{C}(26)-\mathrm{Ru}(1)-\mathrm{C}(24)$ & $67.77(16)$ \\
\hline $\mathrm{C}(26)-\mathrm{Ru}(1)-\mathrm{C}(27)$ & $37.37(16)$ \\
\hline $\mathrm{C}(24)-\mathrm{Ru}(1)-\mathrm{C}(27)$ & $80.00(16)$ \\
\hline $\mathrm{C}(26)-\mathrm{Ru}(1)-\mathrm{C}(25)$ & $38.00(16)$ \\
\hline $\mathrm{C}(24)-\mathrm{Ru}(1)-\mathrm{C}(25)$ & $37.50(16)$ \\
\hline $\mathrm{C}(27)-\mathrm{Ru}(1)-\mathrm{C}(25)$ & $68.12(16)$ \\
\hline $\mathrm{C}(26)-\mathrm{Ru}(1)-\mathrm{C}(22)$ & $67.28(16)$ \\
\hline $\mathrm{C}(24)-\mathrm{Ru}(1)-\mathrm{C}(22)$ & $67.00(16)$ \\
\hline $\mathrm{C}(27)-\mathrm{Ru}(1)-\mathrm{C}(22)$ & $37.51(15)$ \\
\hline $\mathrm{C}(25)-\mathrm{Ru}(1)-\mathrm{C}(22)$ & $79.84(16)$ \\
\hline $\mathrm{C}(26)-\mathrm{Ru}(1)-\mathrm{C}(23)$ & $79.18(16)$ \\
\hline $\mathrm{C}(24)-\mathrm{Ru}(1)-\mathrm{C}(23)$ & $37.59(16)$ \\
\hline $\mathrm{C}(27)-\mathrm{Ru}(1)-\mathrm{C}(23)$ & $66.68(16)$ \\
\hline $\mathrm{C}(25)-\mathrm{Ru}(1)-\mathrm{C}(23)$ & $67.50(16)$ \\
\hline $\mathrm{C}(22)-\mathrm{Ru}(1)-\mathrm{C}(23)$ & $36.21(16)$ \\
\hline $\mathrm{C}(26)-\mathrm{Ru}(1)-\mathrm{P}(1)$ & $90.02(12)$ \\
\hline $\mathrm{C}(24)-\mathrm{Ru}(1)-\mathrm{P}(1)$ & $128.72(12)$ \\
\hline $\mathrm{C}(27)-\mathrm{Ru}(1)-\mathrm{P}(1)$ & $110.10(11)$ \\
\hline $\mathrm{C}(25)-\mathrm{Ru}(1)-\mathrm{P}(1)$ & $97.87(12)$ \\
\hline $\mathrm{C}(22)-\mathrm{Ru}(1)-\mathrm{P}(1)$ & $146.13(12)$ \\
\hline $\mathrm{C}(23)-\mathrm{Ru}(1)-\mathrm{P}(1)$ & $165.32(12)$ \\
\hline $\mathrm{C}(26)-\mathrm{Ru}(1)-\mathrm{Cl}(2)$ & $129.97(12)$ \\
\hline $\mathrm{C}(24)-\mathrm{Ru}(1)-\mathrm{Cl}(2)$ & $143.95(12)$ \\
\hline $\mathrm{C}(27)-\mathrm{Ru}(1)-\mathrm{Cl}(2)$ & $98.81(12)$ \\
\hline $\mathrm{C}(25)-\mathrm{Ru}(1)-\mathrm{Cl}(2)$ & $166.91(12)$ \\
\hline $\mathrm{C}(22)-\mathrm{Ru}(1)-\mathrm{Cl}(2)$ & $90.03(12)$ \\
\hline $\mathrm{C}(23)-\mathrm{Ru}(1)-\mathrm{Cl}(2)$ & $108.66(12)$ \\
\hline $\mathrm{P}(1)-\mathrm{Ru}(1)-\mathrm{Cl}(2)$ & $85.89(4)$ \\
\hline $\mathrm{C}(26)-\mathrm{Ru}(1)-\mathrm{Cl}(1)$ & $141.75(12)$ \\
\hline $\mathrm{C}(24)-\mathrm{Ru}(1)-\mathrm{Cl}(1)$ & $85.62(11)$ \\
\hline $\mathrm{C}(27)-\mathrm{Ru}(1)-\mathrm{Cl}(1)$ & $163.15(11)$ \\
\hline $\mathrm{C}(25)-\mathrm{Ru}(1)-\mathrm{Cl}(1)$ & $105.07(12)$ \\
\hline $\mathrm{C}(22)-\mathrm{Ru}(1)-\mathrm{Cl}(1)$ & $127.71(12)$ \\
\hline $\mathrm{C}(23)-\mathrm{Ru}(1)-\mathrm{Cl}(1)$ & $96.54(11)$ \\
\hline $\mathrm{P}(1)-\mathrm{Ru}(1)-\mathrm{Cl}(1)$ & $85.76(4)$ \\
\hline $\mathrm{Cl}(2)-\mathrm{Ru}(1)-\mathrm{Cl}(1)$ & $87.66(4)$ \\
\hline$C(2)-P(1)-C(3)$ & $97.22(19)$ \\
\hline
\end{tabular}

\begin{tabular}{|c|c|}
\hline $\mathrm{C}(2)-\mathrm{P}(1)-\mathrm{C}(1)$ & $101.55(19)$ \\
\hline $\mathrm{C}(3)-\mathrm{P}(1)-\mathrm{C}(1)$ & $96.91(18)$ \\
\hline $\mathrm{C}(2)-\mathrm{P}(1)-\mathrm{Ru}(1)$ & $115.31(14)$ \\
\hline $\mathrm{C}(3)-\mathrm{P}(1)-\mathrm{Ru}(1)$ & $113.47(14)$ \\
\hline $\mathrm{C}(1)-\mathrm{P}(1)-\mathrm{Ru}(1)$ & $127.15(14)$ \\
\hline $\mathrm{C}(6)-\mathrm{N}(1)-\mathrm{C}(4)$ & $108.5(3)$ \\
\hline $\mathrm{C}(6)-\mathrm{N}(1)-\mathrm{C}(1)$ & $111.8(3)$ \\
\hline $\mathrm{C}(4)-\mathrm{N}(1)-\mathrm{C}(1)$ & $114.9(3)$ \\
\hline $\mathrm{C}(4)-\mathrm{N}(2)-\mathrm{C}(2)$ & $110.0(3)$ \\
\hline $\mathrm{C}(4)-\mathrm{N}(2)-\mathrm{C}(5)$ & $108.6(3)$ \\
\hline$C(2)-N(2)-C(5)$ & $110.8(3)$ \\
\hline $\mathrm{C}(6)-\mathrm{N}(3)-\mathrm{C}(3)$ & $110.3(3)$ \\
\hline $\mathrm{C}(6)-\mathrm{N}(3)-\mathrm{C}(5)$ & $108.3(3)$ \\
\hline $\mathrm{C}(3)-\mathrm{N}(3)-\mathrm{C}(5)$ & $111.3(3)$ \\
\hline $\mathrm{C}(7)-\mathrm{O}(1)-\mathrm{H}(1)$ & $112(4)$ \\
\hline $\mathrm{C}(11)-\mathrm{O}(2)-\mathrm{C}(14)$ & $117.8(5)$ \\
\hline $\mathrm{C}(18)-\mathrm{O}(3)-\mathrm{C}(21)$ & $116.6(4)$ \\
\hline $\mathrm{N}(1)-\mathrm{C}(1)-\mathrm{C}(7)$ & $114.0(3)$ \\
\hline $\mathrm{N}(1)-\mathrm{C}(1)-\mathrm{P}(1)$ & $107.4(3)$ \\
\hline $\mathrm{C}(7)-\mathrm{C}(1)-\mathrm{P}(1)$ & $115.9(3)$ \\
\hline $\mathrm{N}(1)-\mathrm{C}(1)-\mathrm{H}(1 \mathrm{~B})$ & 106.3 \\
\hline $\mathrm{C}(7)-\mathrm{C}(1)-\mathrm{H}(1 \mathrm{~B})$ & 106.3 \\
\hline $\mathrm{P}(1)-\mathrm{C}(1)-\mathrm{H}(1 \mathrm{~B})$ & 106.3 \\
\hline $\mathrm{N}(2)-\mathrm{C}(2)-\mathrm{P}(1)$ & $112.8(3)$ \\
\hline $\mathrm{N}(2)-\mathrm{C}(2)-\mathrm{H}(2 \mathrm{~A})$ & 109.0 \\
\hline $\mathrm{P}(1)-\mathrm{C}(2)-\mathrm{H}(2 \mathrm{~A})$ & 109.0 \\
\hline $\mathrm{N}(2)-\mathrm{C}(2)-\mathrm{H}(2 \mathrm{~B})$ & 109.0 \\
\hline $\mathrm{P}(1)-\mathrm{C}(2)-\mathrm{H}(2 \mathrm{~B})$ & 109.0 \\
\hline $\mathrm{H}(2 \mathrm{~A})-\mathrm{C}(2)-\mathrm{H}(2 \mathrm{~B})$ & 107.8 \\
\hline $\mathrm{N}(3)-\mathrm{C}(3)-\mathrm{P}(1)$ & $112.8(3)$ \\
\hline $\mathrm{N}(3)-\mathrm{C}(3)-\mathrm{H}(3 \mathrm{~A})$ & 109.0 \\
\hline $\mathrm{P}(1)-\mathrm{C}(3)-\mathrm{H}(3 \mathrm{~A})$ & 109.0 \\
\hline $\mathrm{N}(3)-\mathrm{C}(3)-\mathrm{H}(3 \mathrm{~B})$ & 109.0 \\
\hline $\mathrm{P}(1)-\mathrm{C}(3)-\mathrm{H}(3 \mathrm{~B})$ & 109.0 \\
\hline $\mathrm{H}(3 \mathrm{~A})-\mathrm{C}(3)-\mathrm{H}(3 \mathrm{~B})$ & 107.8 \\
\hline $\mathrm{N}(2)-\mathrm{C}(4)-\mathrm{N}(1)$ & $114.4(3)$ \\
\hline $\mathrm{N}(2)-\mathrm{C}(4)-\mathrm{H}(4 \mathrm{~A})$ & 108.7 \\
\hline $\mathrm{N}(1)-\mathrm{C}(4)-\mathrm{H}(4 \mathrm{~A})$ & 108.7 \\
\hline $\mathrm{N}(2)-\mathrm{C}(4)-\mathrm{H}(4 \mathrm{~B})$ & 108.7 \\
\hline $\mathrm{N}(1)-\mathrm{C}(4)-\mathrm{H}(4 \mathrm{~B})$ & 108.7 \\
\hline $\mathrm{H}(4 \mathrm{~A})-\mathrm{C}(4)-\mathrm{H}(4 \mathrm{~B})$ & 107.6 \\
\hline $\mathrm{N}(2)-\mathrm{C}(5)-\mathrm{N}(3)$ & $114.2(3)$ \\
\hline $\mathrm{N}(2)-\mathrm{C}(5)-\mathrm{H}(5 \mathrm{~A})$ & 108.7 \\
\hline $\mathrm{N}(3)-\mathrm{C}(5)-\mathrm{H}(5 \mathrm{~A})$ & 108.7 \\
\hline $\mathrm{N}(2)-\mathrm{C}(5)-\mathrm{H}(5 \mathrm{~B})$ & 108.7 \\
\hline $\mathrm{N}(3)-\mathrm{C}(5)-\mathrm{H}(5 \mathrm{~B})$ & 108.7 \\
\hline $\mathrm{H}(5 \mathrm{~A})-\mathrm{C}(5)-\mathrm{H}(5 \mathrm{~B})$ & 107.6 \\
\hline $\mathrm{N}(1)-\mathrm{C}(6)-\mathrm{N}(3)$ & $113.7(3)$ \\
\hline $\mathrm{N}(1)-\mathrm{C}(6)-\mathrm{H}(6 \mathrm{~A})$ & 108.8 \\
\hline $\mathrm{N}(3)-\mathrm{C}(6)-\mathrm{H}(6 \mathrm{~A})$ & 108.8 \\
\hline $\mathrm{N}(1)-\mathrm{C}(6)-\mathrm{H}(6 \mathrm{~B})$ & 108.8 \\
\hline $\mathrm{N}(3)-\mathrm{C}(6)-\mathrm{H}(6 \mathrm{~B})$ & 108.8 \\
\hline $\mathrm{H}(6 \mathrm{~A})-\mathrm{C}(6)-\mathrm{H}(6 \mathrm{~B})$ & 107.7 \\
\hline $\mathrm{O}(1)-\mathrm{C}(7)-\mathrm{C}(15)$ & $110.4(3)$ \\
\hline $\mathrm{O}(1)-\mathrm{C}(7)-\mathrm{C}(8)$ & $106.1(3)$ \\
\hline$C(15)-C(7)-C(8)$ & $106.4(3)$ \\
\hline
\end{tabular}




\begin{tabular}{|c|c|}
\hline $\mathrm{O}(1)-\mathrm{C}(7)-\mathrm{C}(1)$ & $109.6(3)$ \\
\hline$C(15)-C(7)-C(1)$ & $111.6(3)$ \\
\hline$C(8)-C(7)-C(1)$ & $112.6(3)$ \\
\hline $\mathrm{C}(13)-\mathrm{C}(8)-\mathrm{C}(9)$ & $117.7(4)$ \\
\hline $\mathrm{C}(13)-\mathrm{C}(8)-\mathrm{C}(7)$ & $121.6(4)$ \\
\hline $\mathrm{C}(9)-\mathrm{C}(8)-\mathrm{C}(7)$ & $120.5(4)$ \\
\hline$C(8)-C(9)-C(10)$ & $121.6(5)$ \\
\hline $\mathrm{C}(8)-\mathrm{C}(9)-\mathrm{H}(9 \mathrm{~A})$ & 119.2 \\
\hline $\mathrm{C}(10)-\mathrm{C}(9)-\mathrm{H}(9 \mathrm{~A})$ & 119.2 \\
\hline $\mathrm{C}(11)-\mathrm{C}(10)-\mathrm{C}(9)$ & $119.7(5)$ \\
\hline $\mathrm{C}(11)-\mathrm{C}(10)-\mathrm{H}(10 \mathrm{~A})$ & 120.1 \\
\hline $\mathrm{C}(9)-\mathrm{C}(10)-\mathrm{H}(10 \mathrm{~A})$ & 120.1 \\
\hline $\mathrm{O}(2)-\mathrm{C}(11)-\mathrm{C}(10)$ & $124.5(5)$ \\
\hline $\mathrm{O}(2)-\mathrm{C}(11)-\mathrm{C}(12)$ & $116.0(5)$ \\
\hline$C(10)-C(11)-C(12)$ & $119.5(5)$ \\
\hline $\mathrm{C}(11)-\mathrm{C}(12)-\mathrm{C}(13)$ & $120.2(5)$ \\
\hline $\mathrm{C}(11)-\mathrm{C}(12)-\mathrm{H}(12 \mathrm{~A})$ & 119.9 \\
\hline $\mathrm{C}(13)-\mathrm{C}(12)-\mathrm{H}(12 \mathrm{~A})$ & 119.9 \\
\hline $\mathrm{C}(8)-\mathrm{C}(13)-\mathrm{C}(12)$ & $121.2(5)$ \\
\hline $\mathrm{C}(8)-\mathrm{C}(13)-\mathrm{H}(13 \mathrm{~A})$ & 119.4 \\
\hline $\mathrm{C}(12)-\mathrm{C}(13)-\mathrm{H}(13 \mathrm{~A})$ & 119.4 \\
\hline $\mathrm{O}(2)-\mathrm{C}(14)-\mathrm{H}(14 \mathrm{~A})$ & 109.5 \\
\hline $\mathrm{O}(2)-\mathrm{C}(14)-\mathrm{H}(14 \mathrm{~B})$ & 109.5 \\
\hline $\mathrm{H}(14 \mathrm{~A})-\mathrm{C}(14)-\mathrm{H}(14 \mathrm{~B})$ & 109.5 \\
\hline $\mathrm{O}(2)-\mathrm{C}(14)-\mathrm{H}(14 \mathrm{C})$ & 109.5 \\
\hline $\mathrm{H}(14 \mathrm{~A})-\mathrm{C}(14)-\mathrm{H}(14 \mathrm{C})$ & 109.5 \\
\hline $\mathrm{H}(14 \mathrm{~B})-\mathrm{C}(14)-\mathrm{H}(14 \mathrm{C})$ & 109.5 \\
\hline$C(20)-C(15)-C(16)$ & $117.3(4)$ \\
\hline$C(20)-C(15)-C(7)$ & $122.7(4)$ \\
\hline$C(16)-C(15)-C(7)$ & $119.8(4)$ \\
\hline $\mathrm{C}(17)-\mathrm{C}(16)-\mathrm{C}(15)$ & $121.1(4)$ \\
\hline $\mathrm{C}(17)-\mathrm{C}(16)-\mathrm{H}(16 \mathrm{~A})$ & 119.4 \\
\hline $\mathrm{C}(15)-\mathrm{C}(16)-\mathrm{H}(16 \mathrm{~A})$ & 119.4 \\
\hline$C(16)-C(17)-C(18)$ & $120.6(4)$ \\
\hline $\mathrm{C}(16)-\mathrm{C}(17)-\mathrm{H}(17 \mathrm{~A})$ & 119.7 \\
\hline $\mathrm{C}(18)-\mathrm{C}(17)-\mathrm{H}(17 \mathrm{~A})$ & 119.7 \\
\hline $\mathrm{O}(3)-\mathrm{C}(18)-\mathrm{C}(17)$ & $115.9(4)$ \\
\hline $\mathrm{O}(3)-\mathrm{C}(18)-\mathrm{C}(19)$ & $124.6(4)$ \\
\hline $\mathrm{C}(17)-\mathrm{C}(18)-\mathrm{C}(19)$ & $119.5(4)$ \\
\hline $\mathrm{C}(18)-\mathrm{C}(19)-\mathrm{C}(20)$ & $119.2(4)$ \\
\hline $\mathrm{C}(18)-\mathrm{C}(19)-\mathrm{H}(19 \mathrm{~A})$ & 120.4 \\
\hline $\mathrm{C}(20)-\mathrm{C}(19)-\mathrm{H}(19 \mathrm{~A})$ & 120.4 \\
\hline $\mathrm{C}(15)-\mathrm{C}(20)-\mathrm{C}(19)$ & $122.1(4)$ \\
\hline $\mathrm{C}(15)-\mathrm{C}(20)-\mathrm{H}(20 \mathrm{~A})$ & 118.9 \\
\hline $\mathrm{C}(19)-\mathrm{C}(20)-\mathrm{H}(20 \mathrm{~A})$ & 118.9 \\
\hline $\mathrm{O}(3)-\mathrm{C}(21)-\mathrm{H}(21 \mathrm{~A})$ & 109.5 \\
\hline $\mathrm{O}(3)-\mathrm{C}(21)-\mathrm{H}(21 \mathrm{~B})$ & 109.5 \\
\hline $\mathrm{H}(21 \mathrm{~A})-\mathrm{C}(21)-\mathrm{H}(21 \mathrm{~B})$ & 109.5 \\
\hline $\mathrm{O}(3)-\mathrm{C}(21)-\mathrm{H}(21 \mathrm{C})$ & 109.5 \\
\hline $\mathrm{H}(21 \mathrm{~A})-\mathrm{C}(21)-\mathrm{H}(21 \mathrm{C})$ & 109.5 \\
\hline $\mathrm{H}(21 \mathrm{~B})-\mathrm{C}(21)-\mathrm{H}(21 \mathrm{C})$ & 109.5 \\
\hline $\mathrm{C}(23)-\mathrm{C}(22)-\mathrm{C}(27)$ & $120.1(4)$ \\
\hline$C(23)-C(22)-R u(1)$ & $72.4(3)$ \\
\hline$C(27)-C(22)-R u(1)$ & $69.6(2)$ \\
\hline $\mathrm{C}(23)-\mathrm{C}(22)-\mathrm{H}(22 \mathrm{~A})$ & 119.3 \\
\hline $\mathrm{C}(27)-\mathrm{C}(22)-\mathrm{H}(22 \mathrm{~A})$ & 119.3 \\
\hline
\end{tabular}

\begin{tabular}{|c|c|}
\hline $\mathrm{Ru}(1)-\mathrm{C}(22)-\mathrm{H}(22 \mathrm{~A})$ & 119.3 \\
\hline $\mathrm{C}(22)-\mathrm{C}(23)-\mathrm{C}(24)$ & $119.5(4)$ \\
\hline $\mathrm{C}(22)-\mathrm{C}(23)-\mathrm{Ru}(1)$ & $71.4(3)$ \\
\hline $\mathrm{C}(24)-\mathrm{C}(23)-\mathrm{Ru}(1)$ & $68.6(2)$ \\
\hline $\mathrm{C}(22)-\mathrm{C}(23)-\mathrm{H}(23 \mathrm{~A})$ & 119.4 \\
\hline $\mathrm{C}(24)-\mathrm{C}(23)-\mathrm{H}(23 \mathrm{~A})$ & 119.4 \\
\hline $\mathrm{Ru}(1)-\mathrm{C}(23)-\mathrm{H}(23 \mathrm{~A})$ & 119.4 \\
\hline $\mathrm{C}(25)-\mathrm{C}(24)-\mathrm{C}(23)$ & 121.1(4) \\
\hline$C(25)-C(24)-R u(1)$ & $71.8(2)$ \\
\hline $\mathrm{C}(23)-\mathrm{C}(24)-\mathrm{Ru}(1)$ & $73.8(2)$ \\
\hline $\mathrm{C}(25)-\mathrm{C}(24)-\mathrm{H}(24 \mathrm{~A})$ & 119.1 \\
\hline $\mathrm{C}(23)-\mathrm{C}(24)-\mathrm{H}(24 \mathrm{~A})$ & 119.1 \\
\hline $\mathrm{Ru}(1)-\mathrm{C}(24)-\mathrm{H}(24 \mathrm{~A})$ & 119.1 \\
\hline $\mathrm{C}(24)-\mathrm{C}(25)-\mathrm{C}(26)$ & $118.2(4)$ \\
\hline $\mathrm{C}(24)-\mathrm{C}(25)-\mathrm{Ru}(1)$ & $70.7(2)$ \\
\hline $\mathrm{C}(26)-\mathrm{C}(25)-\mathrm{Ru}(1)$ & $70.1(2)$ \\
\hline $\mathrm{C}(24)-\mathrm{C}(25)-\mathrm{H}(25 \mathrm{~A})$ & 120.3 \\
\hline $\mathrm{C}(26)-\mathrm{C}(25)-\mathrm{H}(25 \mathrm{~A})$ & 120.3 \\
\hline $\mathrm{Ru}(1)-\mathrm{C}(25)-\mathrm{H}(25 \mathrm{~A})$ & 120.3 \\
\hline $\mathrm{C}(27)-\mathrm{C}(26)-\mathrm{C}(25)$ & $121.2(4)$ \\
\hline $\mathrm{C}(27)-\mathrm{C}(26)-\mathrm{Ru}(1)$ & $72.1(2)$ \\
\hline$C(25)-C(26)-R u(1)$ & $71.9(2)$ \\
\hline $\mathrm{C}(27)-\mathrm{C}(26)-\mathrm{H}(26 \mathrm{~A})$ & 118.9 \\
\hline $\mathrm{C}(25)-\mathrm{C}(26)-\mathrm{H}(26 \mathrm{~A})$ & 118.9 \\
\hline $\mathrm{Ru}(1)-\mathrm{C}(26)-\mathrm{H}(26 \mathrm{~A})$ & 118.9 \\
\hline $\mathrm{C}(26)-\mathrm{C}(27)-\mathrm{C}(22)$ & $119.8(4)$ \\
\hline$C(26)-C(27)-R u(1)$ & $70.5(2)$ \\
\hline $\mathrm{C}(22)-\mathrm{C}(27)-\mathrm{Ru}(1)$ & $72.9(2)$ \\
\hline $\mathrm{C}(26)-\mathrm{C}(27)-\mathrm{H}(27 \mathrm{~A})$ & 119.7 \\
\hline $\mathrm{C}(22)-\mathrm{C}(27)-\mathrm{H}(27 \mathrm{~A})$ & 119.7 \\
\hline $\mathrm{Ru}(1)-\mathrm{C}(27)-\mathrm{H}(27 \mathrm{~A})$ & 119.7 \\
\hline $\mathrm{C}(51)-\mathrm{Ru}(2)-\mathrm{C}(49)$ & $67.24(17)$ \\
\hline $\mathrm{C}(51)-\mathrm{Ru}(2)-\mathrm{C}(54)$ & $79.45(17)$ \\
\hline$C(49)-R u(2)-C(54)$ & $37.56(17)$ \\
\hline $\mathrm{C}(51)-\mathrm{Ru}(2)-\mathrm{C}(50)$ & $36.92(17)$ \\
\hline $\mathrm{C}(49)-\mathrm{Ru}(2)-\mathrm{C}(50)$ & $37.37(17)$ \\
\hline $\mathrm{C}(54)-\mathrm{Ru}(2)-\mathrm{C}(50)$ & $67.27(18)$ \\
\hline$C(51)-R u(2)-C(53)$ & $66.25(17)$ \\
\hline $\mathrm{C}(49)-\mathrm{Ru}(2)-\mathrm{C}(53)$ & $67.30(17)$ \\
\hline $\mathrm{C}(54)-\mathrm{Ru}(2)-\mathrm{C}(53)$ & $37.55(17)$ \\
\hline $\mathrm{C}(50)-\mathrm{Ru}(2)-\mathrm{C}(53)$ & $78.55(17)$ \\
\hline $\mathrm{C}(51)-\mathrm{Ru}(2)-\mathrm{C}(52)$ & $37.31(17)$ \\
\hline$C(49)-R u(2)-C(52)$ & $79.79(16)$ \\
\hline $\mathrm{C}(54)-\mathrm{Ru}(2)-\mathrm{C}(52)$ & $67.32(17)$ \\
\hline $\mathrm{C}(50)-\mathrm{Ru}(2)-\mathrm{C}(52)$ & $66.99(16)$ \\
\hline$C(53)-R u(2)-C(52)$ & $36.47(17)$ \\
\hline $\mathrm{C}(51)-\mathrm{Ru}(2)-\mathrm{P}(2)$ & $134.30(13)$ \\
\hline $\mathrm{C}(49)-\mathrm{Ru}(2)-\mathrm{P}(2)$ & $94.38(12)$ \\
\hline $\mathrm{C}(54)-\mathrm{Ru}(2)-\mathrm{P}(2)$ & $111.74(13)$ \\
\hline $\mathrm{C}(50)-\mathrm{Ru}(2)-\mathrm{P}(2)$ & $104.46(12)$ \\
\hline $\mathrm{C}(53)-\mathrm{Ru}(2)-\mathrm{P}(2)$ & $146.24(13)$ \\
\hline $\mathrm{C}(52)-\mathrm{Ru}(2)-\mathrm{P}(2)$ & $171.28(12)$ \\
\hline $\mathrm{C}(51)-\mathrm{Ru}(2)-\mathrm{Cl}(4)$ & $143.68(13)$ \\
\hline $\mathrm{C}(49)-\mathrm{Ru}(2)-\mathrm{Cl}(4)$ & $127.36(13)$ \\
\hline $\mathrm{C}(54)-\mathrm{Ru}(2)-\mathrm{Cl}(4)$ & $95.84(13)$ \\
\hline $\mathrm{C}(50)-\mathrm{Ru}(2)-\mathrm{Cl}(4)$ & $163.11(13)$ \\
\hline
\end{tabular}




\begin{tabular}{|c|c|}
\hline $\mathrm{C}(53)-\mathrm{Ru}(2)-\mathrm{Cl}(4)$ & $88.02(12)$ \\
\hline $\mathrm{C}(52)-\mathrm{Ru}(2)-\mathrm{Cl}(4)$ & $107.57(12)$ \\
\hline $\mathrm{P}(2)-\mathrm{Ru}(2)-\mathrm{Cl}(4)$ & $81.11(4)$ \\
\hline $\mathrm{C}(51)-\mathrm{Ru}(2)-\mathrm{Cl}(3)$ & $86.78(12)$ \\
\hline $\mathrm{C}(49)-\mathrm{Ru}(2)-\mathrm{Cl}(3)$ & $144.16(13)$ \\
\hline $\mathrm{C}(54)-\mathrm{Ru}(2)-\mathrm{Cl}(3)$ & $161.81(13)$ \\
\hline $\mathrm{C}(50)-\mathrm{Ru}(2)-\mathrm{Cl}(3)$ & $107.86(13)$ \\
\hline $\mathrm{C}(53)-\mathrm{Ru}(2)-\mathrm{Cl}(3)$ & $125.33(12)$ \\
\hline $\mathrm{C}(52)-\mathrm{Ru}(2)-\mathrm{Cl}(3)$ & $94.54(12)$ \\
\hline $\mathrm{P}(2)-\mathrm{Ru}(2)-\mathrm{Cl}(3)$ & $86.39(4)$ \\
\hline $\mathrm{Cl}(4)-\mathrm{Ru}(2)-\mathrm{Cl}(3)$ & $88.23(4)$ \\
\hline $\mathrm{C}(30)-\mathrm{P}(2)-\mathrm{C}(29)$ & $98.3(2)$ \\
\hline $\mathrm{C}(30)-\mathrm{P}(2)-\mathrm{C}(28)$ & $100.46(19)$ \\
\hline $\mathrm{C}(29)-\mathrm{P}(2)-\mathrm{C}(28)$ & $96.25(19)$ \\
\hline $\mathrm{C}(30)-\mathrm{P}(2)-\mathrm{Ru}(2)$ & $114.85(14)$ \\
\hline $\mathrm{C}(29)-\mathrm{P}(2)-\mathrm{Ru}(2)$ & $114.35(14)$ \\
\hline $\mathrm{C}(28)-\mathrm{P}(2)-\mathrm{Ru}(2)$ & $127.57(14)$ \\
\hline $\mathrm{C}(33)-\mathrm{N}(4)-\mathrm{C}(28)$ & $114.0(3)$ \\
\hline $\mathrm{C}(33)-\mathrm{N}(4)-\mathrm{C}(31)$ & $108.8(3)$ \\
\hline $\mathrm{C}(28)-\mathrm{N}(4)-\mathrm{C}(31)$ & $111.6(3)$ \\
\hline $\mathrm{C}(29)-\mathrm{N}(5)-\mathrm{C}(32)$ & $111.9(3)$ \\
\hline $\mathrm{C}(29)-\mathrm{N}(5)-\mathrm{C}(31)$ & $109.5(3)$ \\
\hline $\mathrm{C}(32)-\mathrm{N}(5)-\mathrm{C}(31)$ & $108.0(3)$ \\
\hline $\mathrm{C}(30)-\mathrm{N}(6)-\mathrm{C}(32)$ & $111.4(3)$ \\
\hline $\mathrm{C}(30)-\mathrm{N}(6)-\mathrm{C}(33)$ & $110.1(3)$ \\
\hline $\mathrm{C}(32)-\mathrm{N}(6)-\mathrm{C}(33)$ & $108.9(3)$ \\
\hline $\mathrm{C}(34)-\mathrm{O}(4)-\mathrm{H}(4)$ & $106(5)$ \\
\hline $\mathrm{C}(38)-\mathrm{O}(5)-\mathrm{C}(41)$ & $117.2(3)$ \\
\hline $\mathrm{C}(45)-\mathrm{O}(6)-\mathrm{C}(48)$ & $116.9(4)$ \\
\hline$N(4)-C(28)-C(34)$ & $114.4(3)$ \\
\hline $\mathrm{N}(4)-\mathrm{C}(28)-\mathrm{P}(2)$ & $108.4(3)$ \\
\hline $\mathrm{C}(34)-\mathrm{C}(28)-\mathrm{P}(2)$ & $113.9(3)$ \\
\hline $\mathrm{N}(4)-\mathrm{C}(28)-\mathrm{H}(28 \mathrm{~A})$ & 106.5 \\
\hline $\mathrm{C}(34)-\mathrm{C}(28)-\mathrm{H}(28 \mathrm{~A})$ & 106.5 \\
\hline $\mathrm{P}(2)-\mathrm{C}(28)-\mathrm{H}(28 \mathrm{~A})$ & 106.5 \\
\hline $\mathrm{N}(5)-\mathrm{C}(29)-\mathrm{P}(2)$ & $112.8(3)$ \\
\hline $\mathrm{N}(5)-\mathrm{C}(29)-\mathrm{H}(29 \mathrm{~A})$ & 109.0 \\
\hline$P(2)-C(29)-H(29 A)$ & 109.0 \\
\hline $\mathrm{N}(5)-\mathrm{C}(29)-\mathrm{H}(29 \mathrm{~B})$ & 109.0 \\
\hline $\mathrm{P}(2)-\mathrm{C}(29)-\mathrm{H}(29 \mathrm{~B})$ & 109.0 \\
\hline $\mathrm{H}(29 \mathrm{~A})-\mathrm{C}(29)-\mathrm{H}(29 \mathrm{~B})$ & 107.8 \\
\hline $\mathrm{N}(6)-\mathrm{C}(30)-\mathrm{P}(2)$ & $112.3(3)$ \\
\hline $\mathrm{N}(6)-\mathrm{C}(30)-\mathrm{H}(30 \mathrm{~A})$ & 109.2 \\
\hline $\mathrm{P}(2)-\mathrm{C}(30)-\mathrm{H}(30 \mathrm{~A})$ & 109.2 \\
\hline $\mathrm{N}(6)-\mathrm{C}(30)-\mathrm{H}(30 \mathrm{~B})$ & 109.2 \\
\hline $\mathrm{P}(2)-\mathrm{C}(30)-\mathrm{H}(30 \mathrm{~B})$ & 109.2 \\
\hline $\mathrm{H}(30 \mathrm{~A})-\mathrm{C}(30)-\mathrm{H}(30 \mathrm{~B})$ & 107.9 \\
\hline$N(4)-C(31)-N(5)$ & $113.6(3)$ \\
\hline $\mathrm{N}(4)-\mathrm{C}(31)-\mathrm{H}(31 \mathrm{~A})$ & 108.9 \\
\hline $\mathrm{N}(5)-\mathrm{C}(31)-\mathrm{H}(31 \mathrm{~A})$ & 108.9 \\
\hline $\mathrm{N}(4)-\mathrm{C}(31)-\mathrm{H}(31 \mathrm{~B})$ & 108.9 \\
\hline $\mathrm{N}(5)-\mathrm{C}(31)-\mathrm{H}(31 \mathrm{~B})$ & 108.9 \\
\hline $\mathrm{H}(31 \mathrm{~A})-\mathrm{C}(31)-\mathrm{H}(31 \mathrm{~B})$ & 107.7 \\
\hline $\mathrm{N}(6)-\mathrm{C}(32)-\mathrm{N}(5)$ & $114.7(3)$ \\
\hline $\mathrm{N}(6)-\mathrm{C}(32)-\mathrm{H}(32 \mathrm{~A})$ & 108.6 \\
\hline $\mathrm{N}(5)-\mathrm{C}(32)-\mathrm{H}(32 \mathrm{~A})$ & 108.6 \\
\hline
\end{tabular}

\begin{tabular}{|c|c|}
\hline $\mathrm{N}(6)-\mathrm{C}(32)-\mathrm{H}(32 \mathrm{~B})$ & 108.6 \\
\hline $\mathrm{N}(5)-\mathrm{C}(32)-\mathrm{H}(32 \mathrm{~B})$ & 108.6 \\
\hline $\mathrm{H}(32 \mathrm{~A})-\mathrm{C}(32)-\mathrm{H}(32 \mathrm{~B})$ & 107.6 \\
\hline$N(4)-C(33)-N(6)$ & $114.4(4)$ \\
\hline $\mathrm{N}(4)-\mathrm{C}(33)-\mathrm{H}(33 \mathrm{~A})$ & 108.7 \\
\hline $\mathrm{N}(6)-\mathrm{C}(33)-\mathrm{H}(33 \mathrm{~A})$ & 108.7 \\
\hline $\mathrm{N}(4)-\mathrm{C}(33)-\mathrm{H}(33 \mathrm{~B})$ & 108.7 \\
\hline $\mathrm{N}(6)-\mathrm{C}(33)-\mathrm{H}(33 \mathrm{~B})$ & 108.7 \\
\hline $\mathrm{H}(33 \mathrm{~A})-\mathrm{C}(33)-\mathrm{H}(33 \mathrm{~B})$ & 107.6 \\
\hline $\mathrm{O}(4)-\mathrm{C}(34)-\mathrm{C}(42)$ & $110.4(3)$ \\
\hline $\mathrm{O}(4)-\mathrm{C}(34)-\mathrm{C}(35)$ & $105.4(3)$ \\
\hline $\mathrm{C}(42)-\mathrm{C}(34)-\mathrm{C}(35)$ & 107.1(3) \\
\hline $\mathrm{O}(4)-\mathrm{C}(34)-\mathrm{C}(28)$ & $109.5(3)$ \\
\hline$C(42)-C(34)-C(28)$ & $111.9(3)$ \\
\hline $\mathrm{C}(35)-\mathrm{C}(34)-\mathrm{C}(28)$ & $112.3(3)$ \\
\hline $\mathrm{C}(36)-\mathrm{C}(35)-\mathrm{C}(40)$ & $118.2(4)$ \\
\hline$C(36)-C(35)-C(34)$ & $121.0(4)$ \\
\hline$C(40)-C(35)-C(34)$ & $120.7(4)$ \\
\hline $\mathrm{C}(35)-\mathrm{C}(36)-\mathrm{C}(37)$ & $121.2(4)$ \\
\hline $\mathrm{C}(35)-\mathrm{C}(36)-\mathrm{H}(36 \mathrm{~A})$ & 119.4 \\
\hline $\mathrm{C}(37)-\mathrm{C}(36)-\mathrm{H}(36 \mathrm{~A})$ & 119.4 \\
\hline $\mathrm{C}(38)-\mathrm{C}(37)-\mathrm{C}(36)$ & $120.1(4)$ \\
\hline $\mathrm{C}(38)-\mathrm{C}(37)-\mathrm{H}(37 \mathrm{~A})$ & 120.0 \\
\hline $\mathrm{C}(36)-\mathrm{C}(37)-\mathrm{H}(37 \mathrm{~A})$ & 120.0 \\
\hline $\mathrm{O}(5)-\mathrm{C}(38)-\mathrm{C}(37)$ & $124.6(4)$ \\
\hline $\mathrm{O}(5)-\mathrm{C}(38)-\mathrm{C}(39)$ & $115.7(4)$ \\
\hline $\mathrm{C}(37)-\mathrm{C}(38)-\mathrm{C}(39)$ & $119.7(4)$ \\
\hline$C(38)-C(39)-C(40)$ & $120.0(4)$ \\
\hline $\mathrm{C}(38)-\mathrm{C}(39)-\mathrm{H}(39 \mathrm{~A})$ & 120.0 \\
\hline $\mathrm{C}(40)-\mathrm{C}(39)-\mathrm{H}(39 \mathrm{~A})$ & 120.0 \\
\hline $\mathrm{C}(39)-\mathrm{C}(40)-\mathrm{C}(35)$ & $120.9(4)$ \\
\hline $\mathrm{C}(39)-\mathrm{C}(40)-\mathrm{H}(40 \mathrm{~A})$ & 119.6 \\
\hline $\mathrm{C}(35)-\mathrm{C}(40)-\mathrm{H}(40 \mathrm{~A})$ & 119.6 \\
\hline $\mathrm{O}(5)-\mathrm{C}(41)-\mathrm{H}(41 \mathrm{~A})$ & 109.5 \\
\hline $\mathrm{O}(5)-\mathrm{C}(41)-\mathrm{H}(41 \mathrm{~B})$ & 109.5 \\
\hline $\mathrm{H}(41 \mathrm{~A})-\mathrm{C}(41)-\mathrm{H}(41 \mathrm{~B})$ & 109.5 \\
\hline $\mathrm{O}(5)-\mathrm{C}(41)-\mathrm{H}(41 \mathrm{C})$ & 109.5 \\
\hline $\mathrm{H}(41 \mathrm{~A})-\mathrm{C}(41)-\mathrm{H}(41 \mathrm{C})$ & 109.5 \\
\hline $\mathrm{H}(41 \mathrm{~B})-\mathrm{C}(41)-\mathrm{H}(41 \mathrm{C})$ & 109.5 \\
\hline$C(47)-C(42)-C(43)$ & $117.2(4)$ \\
\hline $\mathrm{C}(47)-\mathrm{C}(42)-\mathrm{C}(34)$ & $124.8(4)$ \\
\hline $\mathrm{C}(43)-\mathrm{C}(42)-\mathrm{C}(34)$ & $117.9(4)$ \\
\hline $\mathrm{C}(44)-\mathrm{C}(43)-\mathrm{C}(42)$ & $121.2(4)$ \\
\hline $\mathrm{C}(44)-\mathrm{C}(43)-\mathrm{H}(43 \mathrm{~A})$ & 119.4 \\
\hline $\mathrm{C}(42)-\mathrm{C}(43)-\mathrm{H}(43 \mathrm{~A})$ & 119.4 \\
\hline $\mathrm{C}(43)-\mathrm{C}(44)-\mathrm{C}(45)$ & $121.0(4)$ \\
\hline $\mathrm{C}(43)-\mathrm{C}(44)-\mathrm{H}(44 \mathrm{~A})$ & 119.5 \\
\hline $\mathrm{C}(45)-\mathrm{C}(44)-\mathrm{H}(44 \mathrm{~A})$ & 119.5 \\
\hline $\mathrm{O}(6)-\mathrm{C}(45)-\mathrm{C}(44)$ & $116.4(4)$ \\
\hline $\mathrm{O}(6)-\mathrm{C}(45)-\mathrm{C}(46)$ & $125.4(4)$ \\
\hline$C(44)-C(45)-C(46)$ & $118.2(4)$ \\
\hline$C(47)-C(46)-C(45)$ & $120.5(4)$ \\
\hline $\mathrm{C}(47)-\mathrm{C}(46)-\mathrm{H}(46 \mathrm{~A})$ & 119.7 \\
\hline $\mathrm{C}(45)-\mathrm{C}(46)-\mathrm{H}(46 \mathrm{~A})$ & 119.7 \\
\hline $\mathrm{C}(46)-\mathrm{C}(47)-\mathrm{C}(42)$ & $121.8(4)$ \\
\hline $\mathrm{C}(46)-\mathrm{C}(47)-\mathrm{H}(47 \mathrm{~A})$ & 119.1 \\
\hline
\end{tabular}




$\begin{array}{lclc}\mathrm{C}(42)-\mathrm{C}(47)-\mathrm{H}(47 \mathrm{~A}) & 119.1 & \mathrm{C}(53)-\mathrm{C}(52)-\mathrm{C}(51) & 117.8(4) \\ \mathrm{O}(6)-\mathrm{C}(48)-\mathrm{H}(48 \mathrm{~A}) & 109.5 & \mathrm{C}(53)-\mathrm{C}(52)-\mathrm{Ru}(2) & 71.3(3) \\ \mathrm{O}(6)-\mathrm{C}(48)-\mathrm{H}(48 \mathrm{~B}) & 109.5 & \mathrm{C}(51)-\mathrm{C}(52)-\mathrm{Ru}(2) & 69.0(2) \\ \mathrm{H}(48 \mathrm{~A})-\mathrm{C}(48)-\mathrm{H}(48 \mathrm{~B}) & 109.5 & \mathrm{C}(53)-\mathrm{C}(52)-\mathrm{H}(52 \mathrm{~A}) & 120.5 \\ \mathrm{O}(6)-\mathrm{C}(48)-\mathrm{H}(48 \mathrm{C}) & 109.5 & \mathrm{C}(51)-\mathrm{C}(52)-\mathrm{H}(52 \mathrm{~A}) & 120.5 \\ \mathrm{H}(48 \mathrm{~A})-\mathrm{C}(48)-\mathrm{H}(48 \mathrm{C}) & 109.5 & \mathrm{Ru}(2)-\mathrm{C}(52)-\mathrm{H}(52 \mathrm{~A}) & 120.5 \\ \mathrm{H}(48 \mathrm{~B})-\mathrm{C}(48)-\mathrm{H}(48 \mathrm{C}) & 109.5 & \mathrm{C}(52)-\mathrm{C}(53)-\mathrm{C}(54) & 121.3(4) \\ \mathrm{C}(50)-\mathrm{C}(49)-\mathrm{C}(54) & 120.0(4) & \mathrm{C}(52)-\mathrm{C}(53)-\mathrm{Ru}(2) & 72.3(3) \\ \mathrm{C}(50)-\mathrm{C}(49)-\mathrm{Ru}(2) & 72.3(3) & \mathrm{C}(54)-\mathrm{C}(53)-\mathrm{Ru}(2) & 70.2(3) \\ \mathrm{C}(54)-\mathrm{C}(49)-\mathrm{Ru}(2) & 71.7(3) & \mathrm{C}(52)-\mathrm{C}(53)-\mathrm{H}(53 \mathrm{~A}) & 118.6 \\ \mathrm{C}(50)-\mathrm{C}(49)-\mathrm{H}(49 \mathrm{~A}) & 119.6 & \mathrm{C}(54)-\mathrm{C}(53)-\mathrm{H}(53 \mathrm{~A}) & 118.6 \\ \mathrm{C}(54)-\mathrm{C}(49)-\mathrm{H}(49 \mathrm{~A}) & 119.6 & \mathrm{Ru}(2)-\mathrm{C}(53)-\mathrm{H}(53 \mathrm{~A}) & 118.6 \\ \mathrm{Ru}(2)-\mathrm{C}(49)-\mathrm{H}(49 \mathrm{~A}) & 119.6 & \mathrm{C}(49)-\mathrm{C}(54)-\mathrm{C}(53) & 119.1(4) \\ \mathrm{C}(51)-\mathrm{C}(50)-\mathrm{C}(49) & 119.3(4) & \mathrm{C}(49)-\mathrm{C}(54)-\mathrm{Ru}(2) & 70.7(3) \\ \mathrm{C}(51)-\mathrm{C}(50)-\mathrm{Ru}(2) & 70.2(3) & \mathrm{C}(53)-\mathrm{C}(54)-\mathrm{Ru}(2) & 72.3(3) \\ \mathrm{C}(49)-\mathrm{C}(50)-\mathrm{Ru}(2) & 70.3(3) & \mathrm{C}(49)-\mathrm{C}(54)-\mathrm{H}(54 \mathrm{~A}) & 120.1 \\ \mathrm{C}(51)-\mathrm{C}(50)-\mathrm{H}(50 \mathrm{~A}) & 119.6 & \mathrm{C}(53)-\mathrm{C}(54)-\mathrm{H}(54 \mathrm{~A}) & 120.1 \\ \mathrm{C}(49)-\mathrm{C}(50)-\mathrm{H}(50 \mathrm{~A}) & 119.6 & \mathrm{Ru}(2)-\mathrm{C}(54)-\mathrm{H}(54 \mathrm{~A}) & 120.1 \\ \mathrm{Ru}(2)-\mathrm{C}(50)-\mathrm{H}(50 \mathrm{~A}) & 119.6 & \mathrm{Cl}(3 \mathrm{~S})-\mathrm{C}(1 \mathrm{~S})-\mathrm{Cl}(2 \mathrm{~S}) & 110.4(3) \\ \mathrm{C}(50)-\mathrm{C}(51)-\mathrm{C}(52) & 122.3(4) & \mathrm{Cl}(3 \mathrm{~S})-\mathrm{C}(1 \mathrm{~S})-\mathrm{Cl}(1 \mathrm{~S}) & 110.7(3) \\ \mathrm{C}(50)-\mathrm{C}(51)-\mathrm{Ru}(2) & 72.9(3) & \mathrm{Cl}(2 \mathrm{~S})-\mathrm{C}(1 \mathrm{~S})-\mathrm{Cl}(1 \mathrm{~S}) & 110.0(3) \\ \mathrm{C}(52)-\mathrm{C}(51)-\mathrm{Ru}(2) & 73.7(3) & \mathrm{Cl}(3 \mathrm{~S})-\mathrm{C}(1 \mathrm{~S})-\mathrm{H}(1 \mathrm{SA}) & 108.5 \\ \mathrm{C}(50)-\mathrm{C}(51)-\mathrm{H}(51 \mathrm{~A}) & 118.5 & \mathrm{Cl}(2 \mathrm{~S})-\mathrm{C}(1 \mathrm{~S})-\mathrm{H}(1 \mathrm{SA}) & 108.5 \\ \mathrm{C}(52)-\mathrm{C}(51)-\mathrm{H}(51 \mathrm{~A}) & 118.5 & \mathrm{Cl}(1 \mathrm{~S})-\mathrm{C}(1 \mathrm{~S})-\mathrm{H}(1 \mathrm{SA}) & 108.5 \\ \mathrm{Ru}(2)-\mathrm{C}(51)-\mathrm{H}(51 \mathrm{~A}) & 118.5 & & \end{array}$

Symmetry transformations used to generate equivalent atoms:

Table 12-S. Hydrogen bonds for $\left(\eta^{6}-\mathrm{C}_{6} \mathrm{H}_{6}\right) \mathrm{RuCl}_{2}\left(\mathrm{PTA}-\mathrm{C}\left(\mathrm{C}_{6} \mathrm{H}_{4} \mathrm{OCH}_{3}\right)_{2} \mathrm{OH}\right)\left[\AA\right.$ and $\left.^{\circ}\right]$.

\begin{tabular}{lcccc}
\hline $\mathrm{D}-\mathrm{H} \ldots \mathrm{A}$ & $\mathrm{d}(\mathrm{D}-\mathrm{H})$ & $\mathrm{d}(\mathrm{H} \ldots \mathrm{A})$ & $\mathrm{d}(\mathrm{D} \ldots \mathrm{A})$ & $<(\mathrm{DHA})$ \\
\hline $\mathrm{O}(1)-\mathrm{H}(1) \ldots \mathrm{Cl}(2)$ & $0.75(5)$ & $2.38(5)$ & $3.111(4)$ & $166(5)$ \\
$\mathrm{O}(4)-\mathrm{H}(4) \ldots \mathrm{Cl}(3)$ & $0.74(6)$ & $2.63(6)$ & $3.225(3)$ & $140(6)$
\end{tabular}

Symmetry transformations used to generate equivalent atoms:

Table 13-S. Bond lengths $[\AA ̊]$ and angles $\left[^{\circ}\right]$ for $\left[\left(\eta^{6}-\mathrm{C}_{6} \mathrm{H}_{6}\right) \mathrm{Ru}\left(\mathrm{PTA}-\mathrm{CH}\left(\mathrm{C}_{6} \mathrm{H}_{4} \mathrm{OCH}_{3}\right) \mathrm{OH}\right) \mathrm{Cl}_{2}\right]$.

\begin{tabular}{llll}
\hline $\mathrm{Ru}(1)-\mathrm{C}(19)$ & $2.168(7)$ & $\mathrm{Ru}(2)-\mathrm{C}(38)$ & $2.252(6)$ \\
$\mathrm{Ru}(1)-\mathrm{C}(20)$ & $2.184(7)$ & $\mathrm{Ru}(2)-\mathrm{P}(2)$ & $2.3139(15)$ \\
$\mathrm{Ru}(1)-\mathrm{C}(16)$ & $2.187(6)$ & $\mathrm{Ru}(2)-\mathrm{Cl}(4)$ & $2.4056(15)$ \\
$\mathrm{Ru}(1)-\mathrm{C}(15)$ & $2.202(6)$ & $\mathrm{Ru}(2)-\mathrm{Cl}(3)$ & $2.4227(15)$ \\
$\mathrm{Ru}(1)-\mathrm{C}(18)$ & $2.245(6)$ & $\mathrm{P}(1)-\mathrm{C}(3)$ & $1.832(6)$ \\
$\mathrm{Ru}(1)-\mathrm{C}(17)$ & $2.261(6)$ & $\mathrm{P}(1)-\mathrm{C}(2)$ & $1.851(6)$ \\
$\mathrm{Ru}(1)-\mathrm{P}(1)$ & $2.3108(15)$ & $\mathrm{P}(1)-\mathrm{C}(1)$ & $1.864(6)$ \\
$\mathrm{Ru}(1)-\mathrm{Cl}(1)$ & $2.4122(14)$ & $\mathrm{P}(2)-\mathrm{C}(23)$ & $1.840(6)$ \\
$\mathrm{Ru}(1)-\mathrm{Cl}(2)$ & $2.4229(16)$ & $\mathrm{P}(2)-\mathrm{C}(22)$ & $1.849(6)$ \\
$\mathrm{Ru}(2)-\mathrm{C}(35)$ & $2.179(7)$ & $\mathrm{P}(2)-\mathrm{C}(21)$ & $1.861(6)$ \\
$\mathrm{Ru}(2)-\mathrm{C}(39)$ & $2.182(6)$ & $\mathrm{N}(1)-\mathrm{C}(4)$ & $1.477(7)$ \\
$\mathrm{Ru}(2)-\mathrm{C}(36)$ & $2.184(6)$ & $\mathrm{N}(1)-\mathrm{C}(1)$ & $1.490(7)$ \\
$\mathrm{Ru}(2)-\mathrm{C}(40)$ & $2.195(6)$ & $\mathrm{N}(1)-\mathrm{C}(6)$ & $1.497(8)$ \\
$\mathrm{Ru}(2)-\mathrm{C}(37)$ & $2.240(6)$ & $\mathrm{N}(2)-\mathrm{C}(4)$ & $1.465(7)$
\end{tabular}




\begin{tabular}{|c|c|c|c|}
\hline $\mathrm{N}(2)-\mathrm{C}(2)$ & $1.467(7)$ & $\mathrm{C}(18)-\mathrm{C}(19)$ & $1.417(10)$ \\
\hline $\mathrm{N}(2)-\mathrm{C}(5)$ & $1.471(7)$ & $\mathrm{C}(18)-\mathrm{H}(18 \mathrm{~A})$ & 1.0000 \\
\hline $\mathrm{N}(3)-\mathrm{C}(5)$ & $1.467(8)$ & $\mathrm{C}(19)-\mathrm{C}(20)$ & $1.424(10)$ \\
\hline $\mathrm{N}(3)-\mathrm{C}(6)$ & $1.468(8)$ & $\mathrm{C}(19)-\mathrm{H}(19 \mathrm{~A})$ & 1.0000 \\
\hline $\mathrm{N}(3)-\mathrm{C}(3)$ & $1.469(7)$ & $\mathrm{C}(20)-\mathrm{H}(20 \mathrm{~A})$ & 1.0000 \\
\hline $\mathrm{N}(4)-\mathrm{C}(24)$ & $1.452(8)$ & $\mathrm{C}(21)-\mathrm{C}(27)$ & $1.545(7)$ \\
\hline $\mathrm{N}(4)-\mathrm{C}(26)$ & $1.477(7)$ & $\mathrm{C}(21)-\mathrm{H}(21 \mathrm{~A})$ & 1.0000 \\
\hline $\mathrm{N}(4)-\mathrm{C}(21)$ & $1.478(7)$ & $\mathrm{C}(22)-\mathrm{H}(22 \mathrm{~A})$ & 0.9900 \\
\hline $\mathrm{N}(5)-\mathrm{C}(24)$ & $1.462(8)$ & $\mathrm{C}(22)-\mathrm{H}(22 \mathrm{~B})$ & 0.9900 \\
\hline $\mathrm{N}(5)-\mathrm{C}(25)$ & $1.468(8)$ & $\mathrm{C}(23)-\mathrm{H}(23 \mathrm{~A})$ & 0.9900 \\
\hline $\mathrm{N}(5)-\mathrm{C}(22)$ & $1.473(7)$ & $\mathrm{C}(23)-\mathrm{H}(23 \mathrm{~B})$ & 0.9900 \\
\hline $\mathrm{N}(6)-\mathrm{C}(25)$ & $1.448(8)$ & $\mathrm{C}(24)-\mathrm{H}(24 \mathrm{~A})$ & 0.9900 \\
\hline $\mathrm{N}(6)-\mathrm{C}(26)$ & $1.464(8)$ & $\mathrm{C}(24)-\mathrm{H}(24 \mathrm{~B})$ & 0.9900 \\
\hline $\mathrm{N}(6)-\mathrm{C}(23)$ & $1.475(7)$ & $\mathrm{C}(25)-\mathrm{H}(25 \mathrm{~A})$ & 0.9900 \\
\hline $\mathrm{O}(1)-\mathrm{C}(7)$ & $1.446(7)$ & $\mathrm{C}(25)-\mathrm{H}(25 \mathrm{~B})$ & 0.9900 \\
\hline $\mathrm{O}(1)-\mathrm{H}(1 \mathrm{~A})$ & 0.8400 & $\mathrm{C}(26)-\mathrm{H}(26 \mathrm{~A})$ & 0.9900 \\
\hline $\mathrm{O}(2)-\mathrm{C}(11)$ & $1.367(7)$ & $\mathrm{C}(26)-\mathrm{H}(26 \mathrm{~B})$ & 0.9900 \\
\hline $\mathrm{O}(2)-\mathrm{C}(14)$ & $1.426(8)$ & $\mathrm{C}(27)-\mathrm{C}(28)$ & $1.507(8)$ \\
\hline $\mathrm{O}(3)-\mathrm{C}(27)$ & $1.424(6)$ & $\mathrm{C}(27)-\mathrm{H}(27 \mathrm{~A})$ & 1.0000 \\
\hline $\mathrm{O}(3)-\mathrm{H}(3 \mathrm{~A})$ & 0.8400 & $C(28)-C(29)$ & $1.384(9)$ \\
\hline $\mathrm{O}(4)-\mathrm{C}(31)$ & $1.373(7)$ & $\mathrm{C}(28)-\mathrm{C}(33)$ & $1.387(9)$ \\
\hline $\mathrm{O}(4)-\mathrm{C}(34)$ & $1.416(8)$ & $\mathrm{C}(29)-\mathrm{C}(30)$ & $1.381(8)$ \\
\hline $\mathrm{C}(1)-\mathrm{C}(7)$ & $1.534(8)$ & $\mathrm{C}(29)-\mathrm{H}(29 \mathrm{~A})$ & 0.9500 \\
\hline $\mathrm{C}(1)-\mathrm{H}(1 \mathrm{~B})$ & 1.0000 & $C(30)-C(31)$ & $1.393(9)$ \\
\hline $\mathrm{C}(2)-\mathrm{H}(2 \mathrm{~A})$ & 0.9900 & $\mathrm{C}(30)-\mathrm{H}(30 \mathrm{~A})$ & 0.9500 \\
\hline $\mathrm{C}(2)-\mathrm{H}(2 \mathrm{~B})$ & 0.9900 & $\mathrm{C}(31)-\mathrm{C}(32)$ & $1.372(9)$ \\
\hline $\mathrm{C}(3)-\mathrm{H}(3 \mathrm{~B})$ & 0.9900 & $\mathrm{C}(32)-\mathrm{C}(33)$ & $1.385(9)$ \\
\hline $\mathrm{C}(3)-\mathrm{H}(3 \mathrm{C})$ & 0.9900 & $\mathrm{C}(32)-\mathrm{H}(32 \mathrm{~A})$ & 0.9500 \\
\hline $\mathrm{C}(4)-\mathrm{H}(4 \mathrm{~A})$ & 0.9900 & $\mathrm{C}(33)-\mathrm{H}(33 \mathrm{~A})$ & 0.9500 \\
\hline $\mathrm{C}(4)-\mathrm{H}(4 \mathrm{~B})$ & 0.9900 & $\mathrm{C}(34)-\mathrm{H}(34 \mathrm{~A})$ & 0.9800 \\
\hline $\mathrm{C}(5)-\mathrm{H}(5 \mathrm{~A})$ & 0.9900 & $\mathrm{C}(34)-\mathrm{H}(34 \mathrm{~B})$ & 0.9800 \\
\hline $\mathrm{C}(5)-\mathrm{H}(5 \mathrm{~B})$ & 0.9900 & $\mathrm{C}(34)-\mathrm{H}(34 \mathrm{C})$ & 0.9800 \\
\hline $\mathrm{C}(6)-\mathrm{H}(6 \mathrm{~A})$ & 0.9900 & $C(35)-C(36)$ & $1.403(10)$ \\
\hline $\mathrm{C}(6)-\mathrm{H}(6 \mathrm{~B})$ & 0.9900 & $C(35)-C(40)$ & $1.418(9)$ \\
\hline $\mathrm{C}(7)-\mathrm{C}(8)$ & $1.510(8)$ & $\mathrm{C}(35)-\mathrm{H}(35 \mathrm{~A})$ & 1.0000 \\
\hline $\mathrm{C}(7)-\mathrm{H}(7 \mathrm{~A})$ & 1.0000 & $C(36)-C(37)$ & $1.452(10)$ \\
\hline$C(8)-C(9)$ & $1.381(9)$ & $\mathrm{C}(36)-\mathrm{H}(36 \mathrm{~A})$ & 1.0000 \\
\hline$C(8)-C(13)$ & 1.391(9) & $\mathrm{C}(37)-\mathrm{C}(38)$ & $1.377(9)$ \\
\hline$C(9)-C(10)$ & $1.395(9)$ & $\mathrm{C}(37)-\mathrm{H}(37 \mathrm{~A})$ & 1.0000 \\
\hline $\mathrm{C}(9)-\mathrm{H}(9 \mathrm{~A})$ & 0.9500 & C(38)-C(39) & $1.390(9)$ \\
\hline$C(10)-C(11)$ & $1.387(9)$ & C(38)-H(38A) & 1.0000 \\
\hline $\mathrm{C}(10)-\mathrm{H}(10 \mathrm{~A})$ & 0.9500 & $\mathrm{C}(39)-\mathrm{C}(40)$ & $1.405(9)$ \\
\hline $\mathrm{C}(11)-\mathrm{C}(12)$ & $1.375(9)$ & $\mathrm{C}(39)-\mathrm{H}(39 \mathrm{~A})$ & 1.0000 \\
\hline $\mathrm{C}(12)-\mathrm{C}(13)$ & $1.397(9)$ & $\mathrm{C}(40)-\mathrm{H}(40 \mathrm{~A})$ & 1.0000 \\
\hline $\mathrm{C}(12)-\mathrm{H}(12 \mathrm{~A})$ & 0.9500 & $\mathrm{C}(1 \mathrm{~S})-\mathrm{Cl}(3 \mathrm{~S})$ & $1.737(9)$ \\
\hline $\mathrm{C}(13)-\mathrm{H}(13 \mathrm{~A})$ & 0.9500 & $\mathrm{C}(1 \mathrm{~S})-\mathrm{Cl}(2 \mathrm{~S})$ & $1.777(9)$ \\
\hline $\mathrm{C}(14)-\mathrm{H}(14 \mathrm{~A})$ & 0.9800 & $\mathrm{C}(1 \mathrm{~S})-\mathrm{Cl}(1 \mathrm{~S})$ & $1.779(7)$ \\
\hline $\mathrm{C}(14)-\mathrm{H}(14 \mathrm{~B})$ & 0.9800 & $\mathrm{C}(2 \mathrm{~S})-\mathrm{Cl}(4 \mathrm{~A})$ & $1.641(11)$ \\
\hline $\mathrm{C}(14)-\mathrm{H}(14 \mathrm{C})$ & 0.9800 & $\mathrm{C}(2 \mathrm{~S})-\mathrm{Cl}(6 \mathrm{~S})$ & $1.651(10)$ \\
\hline $\mathrm{C}(15)-\mathrm{C}(16)$ & $1.394(9)$ & $\mathrm{C}(2 \mathrm{~S})-\mathrm{Cl}(5 \mathrm{~S})$ & $1.722(9)$ \\
\hline$C(15)-C(20)$ & $1.408(9)$ & $\mathrm{C}(2 \mathrm{~S})-\mathrm{Cl}(4 \mathrm{~B})$ & $1.985(17)$ \\
\hline $\mathrm{C}(15)-\mathrm{H}(15 \mathrm{~A})$ & 1.0000 & $\mathrm{Cl}(4 \mathrm{~A})-\mathrm{Cl}(4 \mathrm{~B})$ & $1.989(19)$ \\
\hline$C(16)-C(17)$ & $1.414(10)$ & $\mathrm{Cl}(4 \mathrm{~A})-\mathrm{Cl}(8 \mathrm{~B})$ & $2.394(12)$ \\
\hline $\mathrm{C}(16)-\mathrm{H}(16 \mathrm{~A})$ & 1.0000 & $\mathrm{Cl}(4 \mathrm{~B})-\mathrm{Cl}(4 \mathrm{~B}) \# 1$ & $2.129(14)$ \\
\hline $\mathrm{C}(17)-\mathrm{C}(18)$ & $1.376(10)$ & $\mathrm{C}(3 \mathrm{SA})-\mathrm{C}(3 \mathrm{SB})$ & $1.07(3)$ \\
\hline $\mathrm{C}(17)-\mathrm{H}(17 \mathrm{~A})$ & 1.0000 & $\mathrm{C}(3 \mathrm{SA})-\mathrm{Cl}(8 \mathrm{~A})$ & $1.422(16)$ \\
\hline
\end{tabular}




\begin{tabular}{|c|c|}
\hline $\mathrm{C}(3 \mathrm{SA})-\mathrm{Cl}(7 \mathrm{~A})$ & $1.527(16)$ \\
\hline $\mathrm{C}(3 \mathrm{SA})-\mathrm{Cl}(8 \mathrm{~B})$ & $1.762(19)$ \\
\hline $\mathrm{C}(3 \mathrm{SA})-\mathrm{Cl}(9 \mathrm{~A})$ & $1.791(19)$ \\
\hline $\mathrm{C}(3 \mathrm{SA})-\mathrm{Cl}(7 \mathrm{~B})$ & $1.800(18)$ \\
\hline $\mathrm{C}(3 \mathrm{SB})-\mathrm{Cl}(9 \mathrm{~A})$ & $1.30(2)$ \\
\hline $\mathrm{C}(3 \mathrm{SB})-\mathrm{Cl}(8 \mathrm{~A})$ & $1.73(2)$ \\
\hline $\mathrm{C}(3 \mathrm{SB})-\mathrm{Cl}(7 \mathrm{~A})$ & $1.77(2)$ \\
\hline $\mathrm{C}(3 \mathrm{SB})-\mathrm{Cl}(9 \mathrm{~B})$ & $1.788(18)$ \\
\hline $\mathrm{C}(3 \mathrm{SB})-\mathrm{Cl}(8 \mathrm{~B})$ & $1.89(2)$ \\
\hline $\mathrm{C}(3 \mathrm{SB})-\mathrm{Cl}(7 \mathrm{~B})$ & $2.01(2)$ \\
\hline $\mathrm{Cl}(7 \mathrm{~A})-\mathrm{Cl}(7 \mathrm{~B})$ & $0.688(9)$ \\
\hline $\mathrm{Cl}(7 \mathrm{~A})-\mathrm{Cl}(9 \mathrm{~A})$ & $2.433(12)$ \\
\hline $\mathrm{Cl}(8 \mathrm{~A})-\mathrm{Cl}(8 \mathrm{~B})$ & $1.028(6)$ \\
\hline $\mathrm{Cl}(8 \mathrm{~A})-\mathrm{Cl}(9 \mathrm{~A})$ & $2.279(10)$ \\
\hline $\mathrm{Cl}(9 \mathrm{~A})-\mathrm{Cl}(9 \mathrm{~B})$ & $0.667(10)$ \\
\hline $\mathrm{C}(19)-\mathrm{Ru}(1)-\mathrm{C}(20)$ & $38.2(3)$ \\
\hline $\mathrm{C}(19)-\mathrm{Ru}(1)-\mathrm{C}(16)$ & $79.8(2)$ \\
\hline $\mathrm{C}(20)-\mathrm{Ru}(1)-\mathrm{C}(16)$ & $67.4(2)$ \\
\hline $\mathrm{C}(19)-\mathrm{Ru}(1)-\mathrm{C}(15)$ & $67.9(3)$ \\
\hline $\mathrm{C}(20)-\mathrm{Ru}(1)-\mathrm{C}(15)$ & $37.4(2)$ \\
\hline $\mathrm{C}(16)-\mathrm{Ru}(1)-\mathrm{C}(15)$ & $37.0(2)$ \\
\hline $\mathrm{C}(19)-\mathrm{Ru}(1)-\mathrm{C}(18)$ & $37.4(3)$ \\
\hline $\mathrm{C}(20)-\mathrm{Ru}(1)-\mathrm{C}(18)$ & $67.6(3)$ \\
\hline $\mathrm{C}(16)-\mathrm{Ru}(1)-\mathrm{C}(18)$ & $66.3(3)$ \\
\hline $\mathrm{C}(15)-\mathrm{Ru}(1)-\mathrm{C}(18)$ & $78.8(2)$ \\
\hline $\mathrm{C}(19)-\mathrm{Ru}(1)-\mathrm{C}(17)$ & $65.9(3)$ \\
\hline $\mathrm{C}(20)-\mathrm{Ru}(1)-\mathrm{C}(17)$ & $78.4(2)$ \\
\hline $\mathrm{C}(16)-\mathrm{Ru}(1)-\mathrm{C}(17)$ & $37.0(2)$ \\
\hline $\mathrm{C}(15)-\mathrm{Ru}(1)-\mathrm{C}(17)$ & $66.1(2)$ \\
\hline $\mathrm{C}(18)-\mathrm{Ru}(1)-\mathrm{C}(17)$ & $35.5(3)$ \\
\hline $\mathrm{C}(19)-\mathrm{Ru}(1)-\mathrm{P}(1)$ & $115.9(2)$ \\
\hline $\mathrm{C}(20)-\mathrm{Ru}(1)-\mathrm{P}(1)$ & $92.41(17)$ \\
\hline $\mathrm{C}(16)-\mathrm{Ru}(1)-\mathrm{P}(1)$ & $124.39(19)$ \\
\hline $\mathrm{C}(15)-\mathrm{Ru}(1)-\mathrm{P}(1)$ & $96.73(17)$ \\
\hline $\mathrm{C}(18)-\mathrm{Ru}(1)-\mathrm{P}(1)$ & $152.7(2)$ \\
\hline $\mathrm{C}(17)-\mathrm{Ru}(1)-\mathrm{P}(1)$ & $161.36(18)$ \\
\hline $\mathrm{C}(19)-\mathrm{Ru}(1)-\mathrm{Cl}(1)$ & 91.11(19) \\
\hline $\mathrm{C}(20)-\mathrm{Ru}(1)-\mathrm{Cl}(1)$ & 119.13(18) \\
\hline $\mathrm{C}(16)-\mathrm{Ru}(1)-\mathrm{Cl}(1)$ & $152.76(19)$ \\
\hline $\mathrm{C}(15)-\mathrm{Ru}(1)-\mathrm{Cl}(1)$ & $156.55(18)$ \\
\hline $\mathrm{C}(18)-\mathrm{Ru}(1)-\mathrm{Cl}(1)$ & $90.94(18)$ \\
\hline $\mathrm{C}(17)-\mathrm{Ru}(1)-\mathrm{Cl}(1)$ & $115.93(18)$ \\
\hline $\mathrm{P}(1)-\mathrm{Ru}(1)-\mathrm{Cl}(1)$ & $82.70(5)$ \\
\hline $\mathrm{C}(19)-\mathrm{Ru}(1)-\mathrm{Cl}(2)$ & $159.6(2)$ \\
\hline $\mathrm{C}(20)-\mathrm{Ru}(1)-\mathrm{Cl}(2)$ & $151.13(18)$ \\
\hline $\mathrm{C}(16)-\mathrm{Ru}(1)-\mathrm{Cl}(2)$ & $90.87(18)$ \\
\hline $\mathrm{C}(15)-\mathrm{Ru}(1)-\mathrm{Cl}(2)$ & $114.33(18)$ \\
\hline $\mathrm{C}(18)-\mathrm{Ru}(1)-\mathrm{Cl}(2)$ & $122.2(2)$ \\
\hline $\mathrm{C}(17)-\mathrm{Ru}(1)-\mathrm{Cl}(2)$ & $95.7(2)$ \\
\hline $\mathrm{P}(1)-\mathrm{Ru}(1)-\mathrm{Cl}(2)$ & $84.41(5)$ \\
\hline $\mathrm{Cl}(1)-\mathrm{Ru}(1)-\mathrm{Cl}(2)$ & $89.00(6)$ \\
\hline $\mathrm{C}(35)-\mathrm{Ru}(2)-\mathrm{C}(39)$ & $67.8(2)$ \\
\hline $\mathrm{C}(35)-\mathrm{Ru}(2)-\mathrm{C}(36)$ & $37.5(3)$ \\
\hline $\mathrm{C}(39)-\mathrm{Ru}(2)-\mathrm{C}(36)$ & $80.1(2)$ \\
\hline $\mathrm{C}(35)-\mathrm{Ru}(2)-\mathrm{C}(40)$ & $37.8(3)$ \\
\hline
\end{tabular}

\begin{tabular}{|c|c|}
\hline $\mathrm{C}(39)-\mathrm{Ru}(2)-\mathrm{C}(40)$ & $37.4(2)$ \\
\hline $\mathrm{C}(36)-\mathrm{Ru}(2)-\mathrm{C}(40)$ & $67.9(3)$ \\
\hline $\mathrm{C}(35)-\mathrm{Ru}(2)-\mathrm{C}(37)$ & $67.5(3)$ \\
\hline $\mathrm{C}(39)-\mathrm{Ru}(2)-\mathrm{C}(37)$ & $65.8(2)$ \\
\hline $\mathrm{C}(36)-\mathrm{Ru}(2)-\mathrm{C}(37)$ & $38.3(3)$ \\
\hline $\mathrm{C}(40)-\mathrm{Ru}(2)-\mathrm{C}(37)$ & $78.9(2)$ \\
\hline $\mathrm{C}(35)-\mathrm{Ru}(2)-\mathrm{C}(38)$ & $78.8(2)$ \\
\hline $\mathrm{C}(39)-\mathrm{Ru}(2)-\mathrm{C}(38)$ & $36.5(2)$ \\
\hline $\mathrm{C}(36)-\mathrm{Ru}(2)-\mathrm{C}(38)$ & $67.0(2)$ \\
\hline $\mathrm{C}(40)-\mathrm{Ru}(2)-\mathrm{C}(38)$ & $66.4(2)$ \\
\hline $\mathrm{C}(37)-\mathrm{Ru}(2)-\mathrm{C}(38)$ & $35.7(2)$ \\
\hline $\mathrm{C}(35)-\mathrm{Ru}(2)-\mathrm{P}(2)$ & $90.89(18)$ \\
\hline $\mathrm{C}(39)-\mathrm{Ru}(2)-\mathrm{P}(2)$ & $127.79(18)$ \\
\hline $\mathrm{C}(36)-\mathrm{Ru}(2)-\mathrm{P}(2)$ & $111.7(2)$ \\
\hline $\mathrm{C}(40)-\mathrm{Ru}(2)-\mathrm{P}(2)$ & $97.90(17)$ \\
\hline $\mathrm{C}(37)-\mathrm{Ru}(2)-\mathrm{P}(2)$ & 148.98(19) \\
\hline $\mathrm{C}(38)-\mathrm{Ru}(2)-\mathrm{P}(2)$ & $163.82(17)$ \\
\hline $\mathrm{C}(35)-\mathrm{Ru}(2)-\mathrm{Cl}(4)$ & $124.3(2)$ \\
\hline $\mathrm{C}(39)-\mathrm{Ru}(2)-\mathrm{Cl}(4)$ & $147.94(18)$ \\
\hline $\mathrm{C}(36)-\mathrm{Ru}(2)-\mathrm{Cl}(4)$ & $94.6(2)$ \\
\hline $\mathrm{C}(40)-\mathrm{Ru}(2)-\mathrm{Cl}(4)$ & $161.85(18)$ \\
\hline $\mathrm{C}(37)-\mathrm{Ru}(2)-\mathrm{Cl}(4)$ & $90.40(18)$ \\
\hline $\mathrm{C}(38)-\mathrm{Ru}(2)-\mathrm{Cl}(4)$ & $112.42(17)$ \\
\hline $\mathrm{P}(2)-\mathrm{Ru}(2)-\mathrm{Cl}(4)$ & $83.68(5)$ \\
\hline $\mathrm{C}(35)-\mathrm{Ru}(2)-\mathrm{Cl}(3)$ & $146.7(2)$ \\
\hline $\mathrm{C}(39)-\mathrm{Ru}(2)-\mathrm{Cl}(3)$ & $89.08(17)$ \\
\hline $\mathrm{C}(36)-\mathrm{Ru}(2)-\mathrm{Cl}(3)$ & $163.5(2)$ \\
\hline $\mathrm{C}(40)-\mathrm{Ru}(2)-\mathrm{Cl}(3)$ & $110.14(18)$ \\
\hline $\mathrm{C}(37)-\mathrm{Ru}(2)-\mathrm{Cl}(3)$ & $125.62(19)$ \\
\hline $\mathrm{C}(38)-\mathrm{Ru}(2)-\mathrm{Cl}(3)$ & $96.98(17)$ \\
\hline $\mathrm{P}(2)-\mathrm{Ru}(2)-\mathrm{Cl}(3)$ & $84.71(5)$ \\
\hline $\mathrm{Cl}(4)-\mathrm{Ru}(2)-\mathrm{Cl}(3)$ & $88.00(5)$ \\
\hline $\mathrm{C}(3)-\mathrm{P}(1)-\mathrm{C}(2)$ & $98.4(3)$ \\
\hline $\mathrm{C}(3)-\mathrm{P}(1)-\mathrm{C}(1)$ & $98.9(3)$ \\
\hline $\mathrm{C}(2)-\mathrm{P}(1)-\mathrm{C}(1)$ & $99.2(3)$ \\
\hline $\mathrm{C}(3)-\mathrm{P}(1)-\mathrm{Ru}(1)$ & $112.09(19)$ \\
\hline $\mathrm{C}(2)-\mathrm{P}(1)-\mathrm{Ru}(1)$ & $119.6(2)$ \\
\hline $\mathrm{C}(1)-\mathrm{P}(1)-\mathrm{Ru}(1)$ & $123.96(18)$ \\
\hline $\mathrm{C}(23)-\mathrm{P}(2)-\mathrm{C}(22)$ & $97.4(3)$ \\
\hline $\mathrm{C}(23)-\mathrm{P}(2)-\mathrm{C}(21)$ & $99.5(3)$ \\
\hline $\mathrm{C}(22)-\mathrm{P}(2)-\mathrm{C}(21)$ & $99.3(3)$ \\
\hline $\mathrm{C}(23)-\mathrm{P}(2)-\mathrm{Ru}(2)$ & $112.5(2)$ \\
\hline $\mathrm{C}(22)-\mathrm{P}(2)-\mathrm{Ru}(2)$ & $121.1(2)$ \\
\hline $\mathrm{C}(21)-\mathrm{P}(2)-\mathrm{Ru}(2)$ & $122.43(18)$ \\
\hline $\mathrm{C}(4)-\mathrm{N}(1)-\mathrm{C}(1)$ & $112.3(5)$ \\
\hline $\mathrm{C}(4)-\mathrm{N}(1)-\mathrm{C}(6)$ & $108.3(4)$ \\
\hline $\mathrm{C}(1)-\mathrm{N}(1)-\mathrm{C}(6)$ & $111.2(5)$ \\
\hline $\mathrm{C}(4)-\mathrm{N}(2)-\mathrm{C}(2)$ & $110.9(4)$ \\
\hline $\mathrm{C}(4)-\mathrm{N}(2)-\mathrm{C}(5)$ & $108.4(5)$ \\
\hline$C(2)-N(2)-C(5)$ & $111.5(5)$ \\
\hline $\mathrm{C}(5)-\mathrm{N}(3)-\mathrm{C}(6)$ & $109.0(5)$ \\
\hline $\mathrm{C}(5)-\mathrm{N}(3)-\mathrm{C}(3)$ & $111.1(5)$ \\
\hline $\mathrm{C}(6)-\mathrm{N}(3)-\mathrm{C}(3)$ & $110.1(5)$ \\
\hline$C(24)-N(4)-C(26)$ & $108.2(5)$ \\
\hline $\mathrm{C}(24)-\mathrm{N}(4)-\mathrm{C}(21)$ & $113.3(5)$ \\
\hline$C(26)-N(4)-C(21)$ & $111.2(5)$ \\
\hline
\end{tabular}




\begin{tabular}{|c|c|c|c|}
\hline $\mathrm{C}(24)-\mathrm{N}(5)-\mathrm{C}(25)$ & $106.6(5)$ & $\mathrm{C}(8)-\mathrm{C}(9)-\mathrm{H}(9 \mathrm{~A})$ & 119.8 \\
\hline $\mathrm{C}(24)-\mathrm{N}(5)-\mathrm{C}(22)$ & $111.4(5)$ & $\mathrm{C}(10)-\mathrm{C}(9)-\mathrm{H}(9 \mathrm{~A})$ & 119.8 \\
\hline $\mathrm{C}(25)-\mathrm{N}(5)-\mathrm{C}(22)$ & $112.1(5)$ & $\mathrm{C}(11)-\mathrm{C}(10)-\mathrm{C}(9)$ & $120.3(6)$ \\
\hline $\mathrm{C}(25)-\mathrm{N}(6)-\mathrm{C}(26)$ & $108.4(5)$ & $\mathrm{C}(11)-\mathrm{C}(10)-\mathrm{H}(10 \mathrm{~A})$ & 119.8 \\
\hline $\mathrm{C}(25)-\mathrm{N}(6)-\mathrm{C}(23)$ & $110.7(5)$ & $\mathrm{C}(9)-\mathrm{C}(10)-\mathrm{H}(10 \mathrm{~A})$ & 119.8 \\
\hline $\mathrm{C}(26)-\mathrm{N}(6)-\mathrm{C}(23)$ & $109.8(5)$ & $\mathrm{O}(2)-\mathrm{C}(11)-\mathrm{C}(12)$ & $124.3(6)$ \\
\hline $\mathrm{C}(7)-\mathrm{O}(1)-\mathrm{H}(1 \mathrm{~A})$ & 109.5 & $\mathrm{O}(2)-\mathrm{C}(11)-\mathrm{C}(10)$ & $115.7(6)$ \\
\hline $\mathrm{C}(11)-\mathrm{O}(2)-\mathrm{C}(14)$ & $117.8(5)$ & $\mathrm{C}(12)-\mathrm{C}(11)-\mathrm{C}(10)$ & $119.9(6)$ \\
\hline $\mathrm{C}(27)-\mathrm{O}(3)-\mathrm{H}(3 \mathrm{~A})$ & 109.5 & $\mathrm{C}(11)-\mathrm{C}(12)-\mathrm{C}(13)$ & $119.4(6)$ \\
\hline $\mathrm{C}(31)-\mathrm{O}(4)-\mathrm{C}(34)$ & $117.3(5)$ & $\mathrm{C}(11)-\mathrm{C}(12)-\mathrm{H}(12 \mathrm{~A})$ & 120.3 \\
\hline $\mathrm{N}(1)-\mathrm{C}(1)-\mathrm{C}(7)$ & $112.5(5)$ & $\mathrm{C}(13)-\mathrm{C}(12)-\mathrm{H}(12 \mathrm{~A})$ & 120.3 \\
\hline $\mathrm{N}(1)-\mathrm{C}(1)-\mathrm{P}(1)$ & $109.3(4)$ & $\mathrm{C}(8)-\mathrm{C}(13)-\mathrm{C}(12)$ & $121.3(6)$ \\
\hline $\mathrm{C}(7)-\mathrm{C}(1)-\mathrm{P}(1)$ & $113.1(4)$ & $\mathrm{C}(8)-\mathrm{C}(13)-\mathrm{H}(13 \mathrm{~A})$ & 119.3 \\
\hline $\mathrm{N}(1)-\mathrm{C}(1)-\mathrm{H}(1 \mathrm{~B})$ & 107.2 & $\mathrm{C}(12)-\mathrm{C}(13)-\mathrm{H}(13 \mathrm{~A})$ & 119.3 \\
\hline $\mathrm{C}(7)-\mathrm{C}(1)-\mathrm{H}(1 \mathrm{~B})$ & 107.2 & $\mathrm{O}(2)-\mathrm{C}(14)-\mathrm{H}(14 \mathrm{~A})$ & 109.5 \\
\hline $\mathrm{P}(1)-\mathrm{C}(1)-\mathrm{H}(1 \mathrm{~B})$ & 107.2 & $\mathrm{O}(2)-\mathrm{C}(14)-\mathrm{H}(14 \mathrm{~B})$ & 109.5 \\
\hline $\mathrm{N}(2)-\mathrm{C}(2)-\mathrm{P}(1)$ & $111.7(4)$ & $\mathrm{H}(14 \mathrm{~A})-\mathrm{C}(14)-\mathrm{H}(14 \mathrm{~B})$ & 109.5 \\
\hline $\mathrm{N}(2)-\mathrm{C}(2)-\mathrm{H}(2 \mathrm{~A})$ & 109.3 & $\mathrm{O}(2)-\mathrm{C}(14)-\mathrm{H}(14 \mathrm{C})$ & 109.5 \\
\hline $\mathrm{P}(1)-\mathrm{C}(2)-\mathrm{H}(2 \mathrm{~A})$ & 109.3 & $\mathrm{H}(14 \mathrm{~A})-\mathrm{C}(14)-\mathrm{H}(14 \mathrm{C})$ & 109.5 \\
\hline $\mathrm{N}(2)-\mathrm{C}(2)-\mathrm{H}(2 \mathrm{~B})$ & 109.3 & $\mathrm{H}(14 \mathrm{~B})-\mathrm{C}(14)-\mathrm{H}(14 \mathrm{C})$ & 109.5 \\
\hline $\mathrm{P}(1)-\mathrm{C}(2)-\mathrm{H}(2 \mathrm{~B})$ & 109.3 & $\mathrm{C}(16)-\mathrm{C}(15)-\mathrm{C}(20)$ & $119.8(6)$ \\
\hline $\mathrm{H}(2 \mathrm{~A})-\mathrm{C}(2)-\mathrm{H}(2 \mathrm{~B})$ & 108.0 & $\mathrm{C}(16)-\mathrm{C}(15)-\mathrm{Ru}(1)$ & $70.9(3)$ \\
\hline $\mathrm{N}(3)-\mathrm{C}(3)-\mathrm{P}(1)$ & $112.8(4)$ & $\mathrm{C}(20)-\mathrm{C}(15)-\mathrm{Ru}(1)$ & $70.6(4)$ \\
\hline $\mathrm{N}(3)-\mathrm{C}(3)-\mathrm{H}(3 \mathrm{~B})$ & 109.0 & $\mathrm{C}(16)-\mathrm{C}(15)-\mathrm{H}(15 \mathrm{~A})$ & 119.4 \\
\hline $\mathrm{P}(1)-\mathrm{C}(3)-\mathrm{H}(3 \mathrm{~B})$ & 109.0 & $\mathrm{C}(20)-\mathrm{C}(15)-\mathrm{H}(15 \mathrm{~A})$ & 119.4 \\
\hline $\mathrm{N}(3)-\mathrm{C}(3)-\mathrm{H}(3 \mathrm{C})$ & 109.0 & $\mathrm{Ru}(1)-\mathrm{C}(15)-\mathrm{H}(15 \mathrm{~A})$ & 119.4 \\
\hline $\mathrm{P}(1)-\mathrm{C}(3)-\mathrm{H}(3 \mathrm{C})$ & 109.0 & $\mathrm{C}(15)-\mathrm{C}(16)-\mathrm{C}(17)$ & $120.4(6)$ \\
\hline H(3B)-C(3)-H(3C) & 107.8 & $\mathrm{C}(15)-\mathrm{C}(16)-\mathrm{Ru}(1)$ & $72.1(3)$ \\
\hline $\mathrm{N}(2)-\mathrm{C}(4)-\mathrm{N}(1)$ & $114.5(5)$ & $\mathrm{C}(17)-\mathrm{C}(16)-\mathrm{Ru}(1)$ & $74.3(3)$ \\
\hline $\mathrm{N}(2)-\mathrm{C}(4)-\mathrm{H}(4 \mathrm{~A})$ & 108.6 & $\mathrm{C}(15)-\mathrm{C}(16)-\mathrm{H}(16 \mathrm{~A})$ & 119.7 \\
\hline $\mathrm{N}(1)-\mathrm{C}(4)-\mathrm{H}(4 \mathrm{~A})$ & 108.6 & $\mathrm{C}(17)-\mathrm{C}(16)-\mathrm{H}(16 \mathrm{~A})$ & 119.7 \\
\hline $\mathrm{N}(2)-\mathrm{C}(4)-\mathrm{H}(4 \mathrm{~B})$ & 108.6 & $\mathrm{Ru}(1)-\mathrm{C}(16)-\mathrm{H}(16 \mathrm{~A})$ & 119.7 \\
\hline $\mathrm{N}(1)-\mathrm{C}(4)-\mathrm{H}(4 \mathrm{~B})$ & 108.6 & $\mathrm{C}(18)-\mathrm{C}(17)-\mathrm{C}(16)$ & $120.6(6)$ \\
\hline $\mathrm{H}(4 \mathrm{~A})-\mathrm{C}(4)-\mathrm{H}(4 \mathrm{~B})$ & 107.6 & $\mathrm{C}(18)-\mathrm{C}(17)-\mathrm{Ru}(1)$ & $71.6(4)$ \\
\hline $\mathrm{N}(3)-\mathrm{C}(5)-\mathrm{N}(2)$ & $114.7(4)$ & $\mathrm{C}(16)-\mathrm{C}(17)-\mathrm{Ru}(1)$ & $68.6(4)$ \\
\hline $\mathrm{N}(3)-\mathrm{C}(5)-\mathrm{H}(5 \mathrm{~A})$ & 108.6 & $\mathrm{C}(18)-\mathrm{C}(17)-\mathrm{H}(17 \mathrm{~A})$ & 118.6 \\
\hline $\mathrm{N}(2)-\mathrm{C}(5)-\mathrm{H}(5 \mathrm{~A})$ & 108.6 & $\mathrm{C}(16)-\mathrm{C}(17)-\mathrm{H}(17 \mathrm{~A})$ & 118.6 \\
\hline $\mathrm{N}(3)-\mathrm{C}(5)-\mathrm{H}(5 \mathrm{~B})$ & 108.6 & $\mathrm{Ru}(1)-\mathrm{C}(17)-\mathrm{H}(17 \mathrm{~A})$ & 118.6 \\
\hline $\mathrm{N}(2)-\mathrm{C}(5)-\mathrm{H}(5 \mathrm{~B})$ & 108.6 & $\mathrm{C}(17)-\mathrm{C}(18)-\mathrm{C}(19)$ & $119.5(6)$ \\
\hline $\mathrm{H}(5 \mathrm{~A})-\mathrm{C}(5)-\mathrm{H}(5 \mathrm{~B})$ & 107.6 & $\mathrm{C}(17)-\mathrm{C}(18)-\mathrm{Ru}(1)$ & $72.9(4)$ \\
\hline $\mathrm{N}(3)-\mathrm{C}(6)-\mathrm{N}(1)$ & $113.8(5)$ & $\mathrm{C}(19)-\mathrm{C}(18)-\mathrm{Ru}(1)$ & $68.4(4)$ \\
\hline $\mathrm{N}(3)-\mathrm{C}(6)-\mathrm{H}(6 \mathrm{~A})$ & 108.8 & $\mathrm{C}(17)-\mathrm{C}(18)-\mathrm{H}(18 \mathrm{~A})$ & 119.6 \\
\hline $\mathrm{N}(1)-\mathrm{C}(6)-\mathrm{H}(6 \mathrm{~A})$ & 108.8 & $\mathrm{C}(19)-\mathrm{C}(18)-\mathrm{H}(18 \mathrm{~A})$ & 119.6 \\
\hline $\mathrm{N}(3)-\mathrm{C}(6)-\mathrm{H}(6 \mathrm{~B})$ & 108.8 & $\mathrm{Ru}(1)-\mathrm{C}(18)-\mathrm{H}(18 \mathrm{~A})$ & 119.6 \\
\hline $\mathrm{N}(1)-\mathrm{C}(6)-\mathrm{H}(6 \mathrm{~B})$ & 108.8 & $\mathrm{C}(18)-\mathrm{C}(19)-\mathrm{C}(20)$ & $120.2(6)$ \\
\hline $\mathrm{H}(6 \mathrm{~A})-\mathrm{C}(6)-\mathrm{H}(6 \mathrm{~B})$ & 107.7 & $\mathrm{C}(18)-\mathrm{C}(19)-\mathrm{Ru}(1)$ & $74.2(4)$ \\
\hline $\mathrm{O}(1)-\mathrm{C}(7)-\mathrm{C}(8)$ & $108.9(5)$ & $\mathrm{C}(20)-\mathrm{C}(19)-\mathrm{Ru}(1)$ & $71.5(4)$ \\
\hline $\mathrm{O}(1)-\mathrm{C}(7)-\mathrm{C}(1)$ & $107.9(5)$ & $\mathrm{C}(18)-\mathrm{C}(19)-\mathrm{H}(19 \mathrm{~A})$ & 119.7 \\
\hline $\mathrm{C}(8)-\mathrm{C}(7)-\mathrm{C}(1)$ & $112.9(5)$ & $\mathrm{C}(20)-\mathrm{C}(19)-\mathrm{H}(19 \mathrm{~A})$ & 119.7 \\
\hline $\mathrm{O}(1)-\mathrm{C}(7)-\mathrm{H}(7 \mathrm{~A})$ & 109.0 & $\mathrm{Ru}(1)-\mathrm{C}(19)-\mathrm{H}(19 \mathrm{~A})$ & 119.7 \\
\hline $\mathrm{C}(8)-\mathrm{C}(7)-\mathrm{H}(7 \mathrm{~A})$ & 109.0 & $C(15)-C(20)-C(19)$ & $119.1(6)$ \\
\hline $\mathrm{C}(1)-\mathrm{C}(7)-\mathrm{H}(7 \mathrm{~A})$ & 109.0 & $\mathrm{C}(15)-\mathrm{C}(20)-\mathrm{Ru}(1)$ & $72.0(4)$ \\
\hline $\mathrm{C}(9)-\mathrm{C}(8)-\mathrm{C}(13)$ & $118.6(6)$ & $C(19)-C(20)-R u(1)$ & $70.3(4)$ \\
\hline $\mathrm{C}(9)-\mathrm{C}(8)-\mathrm{C}(7)$ & $121.7(6)$ & $\mathrm{C}(15)-\mathrm{C}(20)-\mathrm{H}(20 \mathrm{~A})$ & 120.0 \\
\hline$C(13)-C(8)-C(7)$ & $119.7(6)$ & $\mathrm{C}(19)-\mathrm{C}(20)-\mathrm{H}(20 \mathrm{~A})$ & 120.0 \\
\hline $\mathrm{C}(8)-\mathrm{C}(9)-\mathrm{C}(10)$ & $120.4(6)$ & $\mathrm{Ru}(1)-\mathrm{C}(20)-\mathrm{H}(20 \mathrm{~A})$ & 120.0 \\
\hline
\end{tabular}




\begin{tabular}{|c|c|}
\hline $\mathrm{N}(4)-\mathrm{C}(21)-\mathrm{C}(27)$ & $110.9(5)$ \\
\hline $\mathrm{N}(4)-\mathrm{C}(21)-\mathrm{P}(2)$ & $109.1(4)$ \\
\hline $\mathrm{C}(27)-\mathrm{C}(21)-\mathrm{P}(2)$ & $114.5(4)$ \\
\hline $\mathrm{N}(4)-\mathrm{C}(21)-\mathrm{H}(21 \mathrm{~A})$ & 107.4 \\
\hline $\mathrm{C}(27)-\mathrm{C}(21)-\mathrm{H}(21 \mathrm{~A})$ & 107.4 \\
\hline $\mathrm{P}(2)-\mathrm{C}(21)-\mathrm{H}(21 \mathrm{~A})$ & 107.4 \\
\hline $\mathrm{N}(5)-\mathrm{C}(22)-\mathrm{P}(2)$ & $111.4(4)$ \\
\hline $\mathrm{N}(5)-\mathrm{C}(22)-\mathrm{H}(22 \mathrm{~A})$ & 109.3 \\
\hline $\mathrm{P}(2)-\mathrm{C}(22)-\mathrm{H}(22 \mathrm{~A})$ & 109.3 \\
\hline $\mathrm{N}(5)-\mathrm{C}(22)-\mathrm{H}(22 \mathrm{~B})$ & 109.3 \\
\hline $\mathrm{P}(2)-\mathrm{C}(22)-\mathrm{H}(22 \mathrm{~B})$ & 109.3 \\
\hline $\mathrm{H}(22 \mathrm{~A})-\mathrm{C}(22)-\mathrm{H}(22 \mathrm{~B})$ & 108.0 \\
\hline $\mathrm{N}(6)-\mathrm{C}(23)-\mathrm{P}(2)$ & $112.8(4)$ \\
\hline $\mathrm{N}(6)-\mathrm{C}(23)-\mathrm{H}(23 \mathrm{~A})$ & 109.0 \\
\hline $\mathrm{P}(2)-\mathrm{C}(23)-\mathrm{H}(23 \mathrm{~A})$ & 109.0 \\
\hline $\mathrm{N}(6)-\mathrm{C}(23)-\mathrm{H}(23 \mathrm{~B})$ & 109.0 \\
\hline $\mathrm{P}(2)-\mathrm{C}(23)-\mathrm{H}(23 \mathrm{~B})$ & 109.0 \\
\hline $\mathrm{H}(23 \mathrm{~A})-\mathrm{C}(23)-\mathrm{H}(23 \mathrm{~B})$ & 107.8 \\
\hline $\mathrm{N}(4)-\mathrm{C}(24)-\mathrm{N}(5)$ & $115.0(5)$ \\
\hline $\mathrm{N}(4)-\mathrm{C}(24)-\mathrm{H}(24 \mathrm{~A})$ & 108.5 \\
\hline $\mathrm{N}(5)-\mathrm{C}(24)-\mathrm{H}(24 \mathrm{~A})$ & 108.5 \\
\hline $\mathrm{N}(4)-\mathrm{C}(24)-\mathrm{H}(24 \mathrm{~B})$ & 108.5 \\
\hline $\mathrm{N}(5)-\mathrm{C}(24)-\mathrm{H}(24 \mathrm{~B})$ & 108.5 \\
\hline $\mathrm{H}(24 \mathrm{~A})-\mathrm{C}(24)-\mathrm{H}(24 \mathrm{~B})$ & 107.5 \\
\hline $\mathrm{N}(6)-\mathrm{C}(25)-\mathrm{N}(5)$ & $115.5(5)$ \\
\hline $\mathrm{N}(6)-\mathrm{C}(25)-\mathrm{H}(25 \mathrm{~A})$ & 108.4 \\
\hline $\mathrm{N}(5)-\mathrm{C}(25)-\mathrm{H}(25 \mathrm{~A})$ & 108.4 \\
\hline $\mathrm{N}(6)-\mathrm{C}(25)-\mathrm{H}(25 \mathrm{~B})$ & 108.4 \\
\hline $\mathrm{N}(5)-\mathrm{C}(25)-\mathrm{H}(25 \mathrm{~B})$ & 108.4 \\
\hline $\mathrm{H}(25 \mathrm{~A})-\mathrm{C}(25)-\mathrm{H}(25 \mathrm{~B})$ & 107.5 \\
\hline$N(6)-C(26)-N(4)$ & $114.4(5)$ \\
\hline $\mathrm{N}(6)-\mathrm{C}(26)-\mathrm{H}(26 \mathrm{~A})$ & 108.7 \\
\hline $\mathrm{N}(4)-\mathrm{C}(26)-\mathrm{H}(26 \mathrm{~A})$ & 108.7 \\
\hline $\mathrm{N}(6)-\mathrm{C}(26)-\mathrm{H}(26 \mathrm{~B})$ & 108.7 \\
\hline $\mathrm{N}(4)-\mathrm{C}(26)-\mathrm{H}(26 \mathrm{~B})$ & 108.7 \\
\hline $\mathrm{H}(26 \mathrm{~A})-\mathrm{C}(26)-\mathrm{H}(26 \mathrm{~B})$ & 107.6 \\
\hline $\mathrm{O}(3)-\mathrm{C}(27)-\mathrm{C}(28)$ & $108.7(4)$ \\
\hline $\mathrm{O}(3)-\mathrm{C}(27)-\mathrm{C}(21)$ & $109.6(5)$ \\
\hline $\mathrm{C}(28)-\mathrm{C}(27)-\mathrm{C}(21)$ & $111.7(4)$ \\
\hline $\mathrm{O}(3)-\mathrm{C}(27)-\mathrm{H}(27 \mathrm{~A})$ & 108.9 \\
\hline $\mathrm{C}(28)-\mathrm{C}(27)-\mathrm{H}(27 \mathrm{~A})$ & 108.9 \\
\hline $\mathrm{C}(21)-\mathrm{C}(27)-\mathrm{H}(27 \mathrm{~A})$ & 108.9 \\
\hline $\mathrm{C}(29)-\mathrm{C}(28)-\mathrm{C}(33)$ & 118.1(6) \\
\hline $\mathrm{C}(29)-\mathrm{C}(28)-\mathrm{C}(27)$ & $121.1(5)$ \\
\hline $\mathrm{C}(33)-\mathrm{C}(28)-\mathrm{C}(27)$ & $120.8(6)$ \\
\hline $\mathrm{C}(30)-\mathrm{C}(29)-\mathrm{C}(28)$ & $121.9(6)$ \\
\hline $\mathrm{C}(30)-\mathrm{C}(29)-\mathrm{H}(29 \mathrm{~A})$ & 119.1 \\
\hline $\mathrm{C}(28)-\mathrm{C}(29)-\mathrm{H}(29 \mathrm{~A})$ & 119.1 \\
\hline $\mathrm{C}(29)-\mathrm{C}(30)-\mathrm{C}(31)$ & $119.0(6)$ \\
\hline $\mathrm{C}(29)-\mathrm{C}(30)-\mathrm{H}(30 \mathrm{~A})$ & 120.5 \\
\hline $\mathrm{C}(31)-\mathrm{C}(30)-\mathrm{H}(30 \mathrm{~A})$ & 120.5 \\
\hline $\mathrm{O}(4)-\mathrm{C}(31)-\mathrm{C}(32)$ & $116.3(6)$ \\
\hline $\mathrm{O}(4)-\mathrm{C}(31)-\mathrm{C}(30)$ & $123.8(6)$ \\
\hline $\mathrm{C}(32)-\mathrm{C}(31)-\mathrm{C}(30)$ & $120.0(6)$ \\
\hline $\mathrm{C}(31)-\mathrm{C}(32)-\mathrm{C}(33)$ & $120.3(6)$ \\
\hline $\mathrm{C}(31)-\mathrm{C}(32)-\mathrm{H}(32 \mathrm{~A})$ & 119.8 \\
\hline
\end{tabular}

\begin{tabular}{|c|c|}
\hline $\mathrm{C}(33)-\mathrm{C}(32)-\mathrm{H}(32 \mathrm{~A})$ & 119.8 \\
\hline $\mathrm{C}(32)-\mathrm{C}(33)-\mathrm{C}(28)$ & $120.7(6)$ \\
\hline $\mathrm{C}(32)-\mathrm{C}(33)-\mathrm{H}(33 \mathrm{~A})$ & 119.6 \\
\hline $\mathrm{C}(28)-\mathrm{C}(33)-\mathrm{H}(33 \mathrm{~A})$ & 119.6 \\
\hline $\mathrm{O}(4)-\mathrm{C}(34)-\mathrm{H}(34 \mathrm{~A})$ & 109.5 \\
\hline $\mathrm{O}(4)-\mathrm{C}(34)-\mathrm{H}(34 \mathrm{~B})$ & 109.5 \\
\hline $\mathrm{H}(34 \mathrm{~A})-\mathrm{C}(34)-\mathrm{H}(34 \mathrm{~B})$ & 109.5 \\
\hline $\mathrm{O}(4)-\mathrm{C}(34)-\mathrm{H}(34 \mathrm{C})$ & 109.5 \\
\hline $\mathrm{H}(34 \mathrm{~A})-\mathrm{C}(34)-\mathrm{H}(34 \mathrm{C})$ & 109.5 \\
\hline $\mathrm{H}(34 \mathrm{~B})-\mathrm{C}(34)-\mathrm{H}(34 \mathrm{C})$ & 109.5 \\
\hline$C(36)-C(35)-C(40)$ & $120.3(6)$ \\
\hline$C(36)-C(35)-R u(2)$ & $71.4(4)$ \\
\hline $\mathrm{C}(40)-\mathrm{C}(35)-\mathrm{Ru}(2)$ & $71.7(4)$ \\
\hline $\mathrm{C}(36)-\mathrm{C}(35)-\mathrm{H}(35 \mathrm{~A})$ & 119.3 \\
\hline $\mathrm{C}(40)-\mathrm{C}(35)-\mathrm{H}(35 \mathrm{~A})$ & 119.3 \\
\hline $\mathrm{Ru}(2)-\mathrm{C}(35)-\mathrm{H}(35 \mathrm{~A})$ & 119.3 \\
\hline $\mathrm{C}(35)-\mathrm{C}(36)-\mathrm{C}(37)$ & $118.8(6)$ \\
\hline $\mathrm{C}(35)-\mathrm{C}(36)-\mathrm{Ru}(2)$ & $71.1(4)$ \\
\hline $\mathrm{C}(37)-\mathrm{C}(36)-\mathrm{Ru}(2)$ & $73.0(4)$ \\
\hline $\mathrm{C}(35)-\mathrm{C}(36)-\mathrm{H}(36 \mathrm{~A})$ & 120.4 \\
\hline $\mathrm{C}(37)-\mathrm{C}(36)-\mathrm{H}(36 \mathrm{~A})$ & 120.4 \\
\hline $\mathrm{Ru}(2)-\mathrm{C}(36)-\mathrm{H}(36 \mathrm{~A})$ & 120.4 \\
\hline $\mathrm{C}(38)-\mathrm{C}(37)-\mathrm{C}(36)$ & $119.9(6)$ \\
\hline $\mathrm{C}(38)-\mathrm{C}(37)-\mathrm{Ru}(2)$ & $72.6(4)$ \\
\hline $\mathrm{C}(36)-\mathrm{C}(37)-\mathrm{Ru}(2)$ & $68.8(4)$ \\
\hline $\mathrm{C}(38)-\mathrm{C}(37)-\mathrm{H}(37 \mathrm{~A})$ & 119.3 \\
\hline $\mathrm{C}(36)-\mathrm{C}(37)-\mathrm{H}(37 \mathrm{~A})$ & 119.3 \\
\hline $\mathrm{Ru}(2)-\mathrm{C}(37)-\mathrm{H}(37 \mathrm{~A})$ & 119.3 \\
\hline $\mathrm{C}(37)-\mathrm{C}(38)-\mathrm{C}(39)$ & $120.5(6)$ \\
\hline $\mathrm{C}(37)-\mathrm{C}(38)-\mathrm{Ru}(2)$ & 71.7(4) \\
\hline $\mathrm{C}(39)-\mathrm{C}(38)-\mathrm{Ru}(2)$ & $69.0(3)$ \\
\hline $\mathrm{C}(37)-\mathrm{C}(38)-\mathrm{H}(38 \mathrm{~A})$ & 118.8 \\
\hline $\mathrm{C}(39)-\mathrm{C}(38)-\mathrm{H}(38 \mathrm{~A})$ & 118.8 \\
\hline $\mathrm{Ru}(2)-\mathrm{C}(38)-\mathrm{H}(38 \mathrm{~A})$ & 118.8 \\
\hline $\mathrm{C}(38)-\mathrm{C}(39)-\mathrm{C}(40)$ & $121.1(6)$ \\
\hline $\mathrm{C}(38)-\mathrm{C}(39)-\mathrm{Ru}(2)$ & $74.5(4)$ \\
\hline $\mathrm{C}(40)-\mathrm{C}(39)-\mathrm{Ru}(2)$ & $71.8(4)$ \\
\hline $\mathrm{C}(38)-\mathrm{C}(39)-\mathrm{H}(39 \mathrm{~A})$ & 119.2 \\
\hline $\mathrm{C}(40)-\mathrm{C}(39)-\mathrm{H}(39 \mathrm{~A})$ & 119.2 \\
\hline $\mathrm{Ru}(2)-\mathrm{C}(39)-\mathrm{H}(39 \mathrm{~A})$ & 119.2 \\
\hline $\mathrm{C}(39)-\mathrm{C}(40)-\mathrm{C}(35)$ & 119.1(6) \\
\hline $\mathrm{C}(39)-\mathrm{C}(40)-\mathrm{Ru}(2)$ & $70.8(4)$ \\
\hline $\mathrm{C}(35)-\mathrm{C}(40)-\mathrm{Ru}(2)$ & $70.5(4)$ \\
\hline $\mathrm{C}(39)-\mathrm{C}(40)-\mathrm{H}(40 \mathrm{~A})$ & 119.8 \\
\hline $\mathrm{C}(35)-\mathrm{C}(40)-\mathrm{H}(40 \mathrm{~A})$ & 119.8 \\
\hline $\mathrm{Ru}(2)-\mathrm{C}(40)-\mathrm{H}(40 \mathrm{~A})$ & 119.8 \\
\hline $\mathrm{Cl}(3 \mathrm{~S})-\mathrm{C}(1 \mathrm{~S})-\mathrm{Cl}(2 \mathrm{~S})$ & $109.3(4)$ \\
\hline $\mathrm{Cl}(3 \mathrm{~S})-\mathrm{C}(1 \mathrm{~S})-\mathrm{Cl}(1 \mathrm{~S})$ & $110.6(5)$ \\
\hline $\mathrm{Cl}(2 \mathrm{~S})-\mathrm{C}(1 \mathrm{~S})-\mathrm{Cl}(1 \mathrm{~S})$ & $108.6(4)$ \\
\hline $\mathrm{Cl}(4 \mathrm{~A})-\mathrm{C}(2 \mathrm{~S})-\mathrm{Cl}(6 \mathrm{~S})$ & $118.8(6)$ \\
\hline $\mathrm{Cl}(4 \mathrm{~A})-\mathrm{C}(2 \mathrm{~S})-\mathrm{Cl}(5 \mathrm{~S})$ & $118.2(6)$ \\
\hline $\mathrm{Cl}(6 \mathrm{~S})-\mathrm{C}(2 \mathrm{~S})-\mathrm{Cl}(5 \mathrm{~S})$ & $117.4(6)$ \\
\hline $\mathrm{Cl}(4 \mathrm{~A})-\mathrm{C}(2 \mathrm{~S})-\mathrm{Cl}(4 \mathrm{~B})$ & $65.8(7)$ \\
\hline $\mathrm{Cl}(6 \mathrm{~S})-\mathrm{C}(2 \mathrm{~S})-\mathrm{Cl}(4 \mathrm{~B})$ & $87.9(6)$ \\
\hline $\mathrm{Cl}(5 \mathrm{~S})-\mathrm{C}(2 \mathrm{~S})-\mathrm{Cl}(4 \mathrm{~B})$ & $93.0(7)$ \\
\hline $\mathrm{C}(2 \mathrm{~S})-\mathrm{Cl}(4 \mathrm{~A})-\mathrm{Cl}(4 \mathrm{~B})$ & $65.5(6)$ \\
\hline
\end{tabular}




\begin{tabular}{|c|c|c|c|}
\hline $\mathrm{C}(2 \mathrm{~S})-\mathrm{Cl}(4 \mathrm{~A})-\mathrm{Cl}(8 \mathrm{~B})$ & $129.8(7)$ & $\mathrm{Cl}(8 \mathrm{~A})-\mathrm{C}(3 \mathrm{SB})-\mathrm{Cl}(7 \mathrm{~B})$ & $109.6(13)$ \\
\hline $\mathrm{Cl}(4 \mathrm{~B})-\mathrm{Cl}(4 \mathrm{~A})-\mathrm{Cl}(8 \mathrm{~B})$ & $156.8(6)$ & $\mathrm{Cl}(7 \mathrm{~A})-\mathrm{C}(3 \mathrm{SB})-\mathrm{Cl}(7 \mathrm{~B})$ & $19.7(4)$ \\
\hline $\mathrm{C}(2 \mathrm{~S})-\mathrm{Cl}(4 \mathrm{~B})-\mathrm{Cl}(4 \mathrm{~A})$ & $48.8(5)$ & Cl(9B)-C(3SB)-Cl(7B) & $125.8(12)$ \\
\hline $\mathrm{C}(2 \mathrm{~S})-\mathrm{Cl}(4 \mathrm{~B})-\mathrm{Cl}(4 \mathrm{~B}) \# 1$ & $152.0(16)$ & $\mathrm{Cl}(8 \mathrm{~B})-\mathrm{C}(3 \mathrm{SB})-\mathrm{Cl}(7 \mathrm{~B})$ & $94.5(9)$ \\
\hline $\mathrm{Cl}(4 \mathrm{~A})-\mathrm{Cl}(4 \mathrm{~B})-\mathrm{Cl}(4 \mathrm{~B}) \# 1$ & $106.4(14)$ & $\mathrm{Cl}(7 \mathrm{~B})-\mathrm{Cl}(7 \mathrm{~A})-\mathrm{C}(3 \mathrm{SA})$ & $102.0(15)$ \\
\hline $\mathrm{C}(3 \mathrm{SB})-\mathrm{C}(3 \mathrm{SA})-\mathrm{Cl}(8 \mathrm{~A})$ & $86.6(16)$ & $\mathrm{Cl}(7 \mathrm{~B})-\mathrm{Cl}(7 \mathrm{~A})-\mathrm{C}(3 \mathrm{SB})$ & $100.1(14)$ \\
\hline $\mathrm{C}(3 \mathrm{SB})-\mathrm{C}(3 \mathrm{SA})-\mathrm{Cl}(7 \mathrm{~A})$ & $83.8(16)$ & $\mathrm{C}(3 \mathrm{SA})-\mathrm{Cl}(7 \mathrm{~A})-\mathrm{C}(3 \mathrm{SB})$ & $36.9(9)$ \\
\hline $\mathrm{Cl}(8 \mathrm{~A})-\mathrm{C}(3 \mathrm{SA})-\mathrm{Cl}(7 \mathrm{~A})$ & $162.8(16)$ & $\mathrm{Cl}(7 \mathrm{~B})-\mathrm{Cl}(7 \mathrm{~A})-\mathrm{Cl}(9 \mathrm{~A})$ & $131.0(14)$ \\
\hline $\mathrm{C}(3 \mathrm{SB})-\mathrm{C}(3 \mathrm{SA})-\mathrm{Cl}(8 \mathrm{~B})$ & $79.7(16)$ & $\mathrm{C}(3 \mathrm{SA})-\mathrm{Cl}(7 \mathrm{~A})-\mathrm{Cl}(9 \mathrm{~A})$ & $47.2(7)$ \\
\hline $\mathrm{Cl}(8 \mathrm{~A})-\mathrm{C}(3 \mathrm{SA})-\mathrm{Cl}(8 \mathrm{~B})$ & $35.7(5)$ & $\mathrm{C}(3 \mathrm{SB})-\mathrm{Cl}(7 \mathrm{~A})-\mathrm{Cl}(9 \mathrm{~A})$ & $31.2(6)$ \\
\hline $\mathrm{Cl}(7 \mathrm{~A})-\mathrm{C}(3 \mathrm{SA})-\mathrm{Cl}(8 \mathrm{~B})$ & $127.9(13)$ & $\mathrm{Cl}(7 \mathrm{~A})-\mathrm{Cl}(7 \mathrm{~B})-\mathrm{C}(3 \mathrm{SA})$ & $56.1(12)$ \\
\hline C(3SB)-C(3SA)-Cl(9A) & $46.0(13)$ & $\mathrm{Cl}(7 \mathrm{~A})-\mathrm{Cl}(7 \mathrm{~B})-\mathrm{C}(3 \mathrm{SB})$ & $60.1(12)$ \\
\hline $\mathrm{Cl}(8 \mathrm{~A})-\mathrm{C}(3 \mathrm{SA})-\mathrm{Cl}(9 \mathrm{~A})$ & $89.6(9)$ & $\mathrm{C}(3 \mathrm{SA})-\mathrm{Cl}(7 \mathrm{~B})-\mathrm{C}(3 \mathrm{SB})$ & $32.0(8)$ \\
\hline $\mathrm{Cl}(7 \mathrm{~A})-\mathrm{C}(3 \mathrm{SA})-\mathrm{Cl}(9 \mathrm{~A})$ & $94.0(10)$ & $\mathrm{Cl}(8 \mathrm{~B})-\mathrm{Cl}(8 \mathrm{~A})-\mathrm{C}(3 \mathrm{SA})$ & $90.5(9)$ \\
\hline $\mathrm{Cl}(8 \mathrm{~B})-\mathrm{C}(3 \mathrm{SA})-\mathrm{Cl}(9 \mathrm{~A})$ & $108.2(10)$ & $\mathrm{Cl}(8 \mathrm{~B})-\mathrm{Cl}(8 \mathrm{~A})-\mathrm{C}(3 \mathrm{SB})$ & $82.5(8)$ \\
\hline $\mathrm{C}(3 \mathrm{SB})-\mathrm{C}(3 \mathrm{SA})-\mathrm{Cl}(7 \mathrm{~B})$ & $84.7(15)$ & $\mathrm{C}(3 \mathrm{SA})-\mathrm{Cl}(8 \mathrm{~A})-\mathrm{C}(3 \mathrm{SB})$ & $38.1(9)$ \\
\hline $\mathrm{Cl}(8 \mathrm{~A})-\mathrm{C}(3 \mathrm{SA})-\mathrm{Cl}(7 \mathrm{~B})$ & $142.6(14)$ & $\mathrm{Cl}(8 \mathrm{~B})-\mathrm{Cl}(8 \mathrm{~A})-\mathrm{Cl}(9 \mathrm{~A})$ & $115.7(6)$ \\
\hline $\mathrm{Cl}(7 \mathrm{~A})-\mathrm{C}(3 \mathrm{SA})-\mathrm{Cl}(7 \mathrm{~B})$ & $21.9(4)$ & $\mathrm{C}(3 \mathrm{SA})-\mathrm{Cl}(8 \mathrm{~A})-\mathrm{Cl}(9 \mathrm{~A})$ & $51.8(8)$ \\
\hline $\mathrm{Cl}(8 \mathrm{~B})-\mathrm{C}(3 \mathrm{SA})-\mathrm{Cl}(7 \mathrm{~B})$ & $106.9(11)$ & $\mathrm{C}(3 \mathrm{SB})-\mathrm{Cl}(8 \mathrm{~A})-\mathrm{Cl}(9 \mathrm{~A})$ & $34.5(6)$ \\
\hline $\mathrm{Cl}(9 \mathrm{~A})-\mathrm{C}(3 \mathrm{SA})-\mathrm{Cl}(7 \mathrm{~B})$ & 109.4(10) & $\mathrm{Cl}(8 \mathrm{~A})-\mathrm{Cl}(8 \mathrm{~B})-\mathrm{C}(3 \mathrm{SA})$ & $53.8(7)$ \\
\hline $\mathrm{C}(3 \mathrm{SA})-\mathrm{C}(3 \mathrm{SB})-\mathrm{Cl}(9 \mathrm{~A})$ & $98(2)$ & $\mathrm{Cl}(8 \mathrm{~A})-\mathrm{Cl}(8 \mathrm{~B})-\mathrm{C}(3 \mathrm{SB})$ & $64.9(8)$ \\
\hline $\mathrm{C}(3 \mathrm{SA})-\mathrm{C}(3 \mathrm{SB})-\mathrm{Cl}(8 \mathrm{~A})$ & $55.3(15)$ & $\mathrm{C}(3 \mathrm{SA})-\mathrm{Cl}(8 \mathrm{~B})-\mathrm{C}(3 \mathrm{SB})$ & $33.8(8)$ \\
\hline $\mathrm{Cl}(9 \mathrm{~A})-\mathrm{C}(3 \mathrm{SB})-\mathrm{Cl}(8 \mathrm{~A})$ & $96.7(14)$ & $\mathrm{Cl}(8 \mathrm{~A})-\mathrm{Cl}(8 \mathrm{~B})-\mathrm{Cl}(4 \mathrm{~A})$ & $152.3(5)$ \\
\hline $\mathrm{C}(3 \mathrm{SA})-\mathrm{C}(3 \mathrm{SB})-\mathrm{Cl}(7 \mathrm{~A})$ & $59.2(14)$ & $\mathrm{C}(3 \mathrm{SA})-\mathrm{Cl}(8 \mathrm{~B})-\mathrm{Cl}(4 \mathrm{~A})$ & $98.5(6)$ \\
\hline $\mathrm{Cl}(9 \mathrm{~A})-\mathrm{C}(3 \mathrm{SB})-\mathrm{Cl}(7 \mathrm{~A})$ & $104.0(14)$ & $\mathrm{C}(3 \mathrm{SB})-\mathrm{Cl}(8 \mathrm{~B})-\mathrm{Cl}(4 \mathrm{~A})$ & $91.3(6)$ \\
\hline $\mathrm{Cl}(8 \mathrm{~A})-\mathrm{C}(3 \mathrm{SB})-\mathrm{Cl}(7 \mathrm{~A})$ & $113.1(16)$ & $\mathrm{Cl}(9 \mathrm{~B})-\mathrm{Cl}(9 \mathrm{~A})-\mathrm{C}(3 \mathrm{SB})$ & $128(2)$ \\
\hline $\mathrm{C}(3 \mathrm{SA})-\mathrm{C}(3 \mathrm{SB})-\mathrm{Cl}(9 \mathrm{~B})$ & $114(2)$ & $\mathrm{Cl}(9 \mathrm{~B})-\mathrm{Cl}(9 \mathrm{~A})-\mathrm{C}(3 \mathrm{SA})$ & $162(2)$ \\
\hline $\mathrm{Cl}(9 \mathrm{~A})-\mathrm{C}(3 \mathrm{SB})-\mathrm{Cl}(9 \mathrm{~B})$ & $17.1(7)$ & $\mathrm{C}(3 \mathrm{SB})-\mathrm{Cl}(9 \mathrm{~A})-\mathrm{C}(3 \mathrm{SA})$ & $36.2(14)$ \\
\hline $\mathrm{Cl}(8 \mathrm{~A})-\mathrm{C}(3 \mathrm{SB})-\mathrm{Cl}(9 \mathrm{~B})$ & 109.1(11) & $\mathrm{Cl}(9 \mathrm{~B})-\mathrm{Cl}(9 \mathrm{~A})-\mathrm{Cl}(8 \mathrm{~A})$ & $147.5(19)$ \\
\hline $\mathrm{Cl}(7 \mathrm{~A})-\mathrm{C}(3 \mathrm{SB})-\mathrm{Cl}(9 \mathrm{~B})$ & $108.2(11)$ & $\mathrm{C}(3 \mathrm{SB})-\mathrm{Cl}(9 \mathrm{~A})-\mathrm{Cl}(8 \mathrm{~A})$ & $48.8(11)$ \\
\hline $\mathrm{C}(3 \mathrm{SA})-\mathrm{C}(3 \mathrm{SB})-\mathrm{Cl}(8 \mathrm{~B})$ & $66.5(15)$ & $\mathrm{C}(3 \mathrm{SA})-\mathrm{Cl}(9 \mathrm{~A})-\mathrm{Cl}(8 \mathrm{~A})$ & $38.6(5)$ \\
\hline $\mathrm{Cl}(9 \mathrm{~A})-\mathrm{C}(3 \mathrm{SB})-\mathrm{Cl}(8 \mathrm{~B})$ & $127.8(15)$ & $\mathrm{Cl}(9 \mathrm{~B})-\mathrm{Cl}(9 \mathrm{~A})-\mathrm{Cl}(7 \mathrm{~A})$ & $126.3(19)$ \\
\hline $\mathrm{Cl}(8 \mathrm{~A})-\mathrm{C}(3 \mathrm{SB})-\mathrm{Cl}(8 \mathrm{~B})$ & $32.6(4)$ & $\mathrm{C}(3 \mathrm{SB})-\mathrm{Cl}(9 \mathrm{~A})-\mathrm{Cl}(7 \mathrm{~A})$ & $44.8(11)$ \\
\hline $\mathrm{Cl}(7 \mathrm{~A})-\mathrm{C}(3 \mathrm{SB})-\mathrm{Cl}(8 \mathrm{~B})$ & $107.8(12)$ & $\mathrm{C}(3 \mathrm{SA})-\mathrm{Cl}(9 \mathrm{~A})-\mathrm{Cl}(7 \mathrm{~A})$ & $38.7(5)$ \\
\hline $\mathrm{Cl}(9 \mathrm{~B})-\mathrm{C}(3 \mathrm{SB})-\mathrm{Cl}(8 \mathrm{~B})$ & $136.6(11)$ & $\mathrm{Cl}(8 \mathrm{~A})-\mathrm{Cl}(9 \mathrm{~A})-\mathrm{Cl}(7 \mathrm{~A})$ & $76.4(3)$ \\
\hline $\mathrm{C}(3 \mathrm{SA})-\mathrm{C}(3 \mathrm{SB})-\mathrm{Cl}(7 \mathrm{~B})$ & $63.3(13)$ & Cl(9A)-Cl(9B)-C(3SB) & $35.0(17)$ \\
\hline $\mathrm{Cl}(9 \mathrm{~A})-\mathrm{C}(3 \mathrm{SB})-\mathrm{Cl}(7 \mathrm{~B})$ & $123.6(15)$ & & \\
\hline
\end{tabular}

Symmetry transformations used to generate equivalent atoms: $\# 1-\mathrm{x}+2,-\mathrm{y}+1,-\mathrm{z}$ 